\title{
Direct Electrochemical Defluorinative Carboxylation of gem- Difluoroalkenes with Carbon Dioxide
}

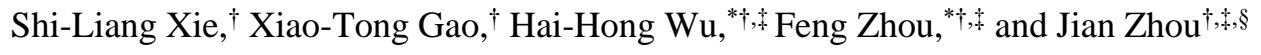 \\ ${ }^{\dagger}$ Shanghai Key Laboratory of Green Chemistry and Chemical Process and ${ }^{\ddagger}$ Shanghai Engineering Research Center of Molecular \\ Therapeutics and New Drug Development, East China Normal University, 3663N Zhongshan Road, Shanghai 200062, China.

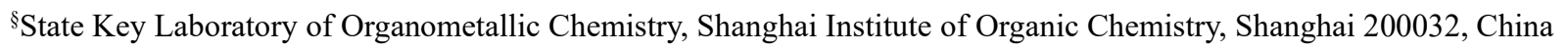 \\ E-mail: hhwu@chem.ecnu.edu.cn; fzhou@chem.ecnu.edu.cn
}

\section{Supporting Information}

\begin{tabular}{|l|c|}
\hline \multicolumn{1}{|c|}{ Table of Contents } & Page \\
\hline 1. General information & S1 \\
\hline 2. Photographic guide for electrochemical reaction & S2-S4 \\
\hline 3. Preparation and characterization of gem-difluoroalkenes & S5-S6 \\
\hline 4. General procedure for the electrolysis & S6-S13 \\
\hline 5. Product elaboration & S13-S14 \\
\hline 6. Cyclic voltammetry & $\mathrm{S} 15$ \\
\hline 7. NMR spectra & S16-S93 \\
\hline
\end{tabular}




\section{General information}

Reactions were monitored by thin layer chromatography using UV light to visualize the course of reaction. Purification of reaction products was carried out by flash chromatography on silica gel. Chemical yields refer to pure isolated substances. Infrared (IR) spectra were obtained using a Bruker tensor 27 infrared spectrometer. Low-resolution mass spectrometry (EI) was recorded with a Shimadzu GCMS-QP2010. High-resolution mass spectrometry (EI) was recorded with an Agilent 5975 C MSD. High-resolution mass spectrometry (ESI) was recorded with a maXis impact 282001.00122. ${ }^{1} \mathrm{H}$ NMR, ${ }^{13} \mathrm{C}$ NMR and ${ }^{19} \mathrm{~F}$ NMR spectra were obtained using Bruker DPX-400 MHz Spectrometer. Chemical shifts were reported in ppm with TMS as the internal standard. The following abbreviations were used to designate chemical shift multiplicities: $\mathrm{s}=$ singlet, $\mathrm{d}=$ doublet, $\mathrm{t}=$ triplet, $\mathrm{q}=$ quartet, $\mathrm{h}=$ heptet, $\mathrm{m}$ $=$ multiplet, $\mathrm{br}=$ broad.

Unless mentioned, all reactions were performed under an atmosphere of $\mathrm{N}_{2}$ and carried out with dry solvents. ${ }^{n} \mathrm{Bu} 4 \mathrm{NI}, \mathrm{Et}_{4} \mathrm{NI},{ }^{n} \mathrm{Bu} 4 \mathrm{NBr},{ }^{n} \mathrm{Bu}_{4} \mathrm{NBF}_{4},{ }^{n} \mathrm{Bu}_{4} \mathrm{NClO}_{4}$ were purchased from Macklin and used as received. $\mathrm{CO}_{2}(99.999 \%)$ was commercially available.

\section{List of abbreviation:}

\begin{tabular}{ccc}
\hline Entry & Chemical name & Abbreviation \\
\hline 1 & Petroleum ether & $\mathrm{PE}$ \\
2 & Ethyl acetate & EtOAc \\
3 & $N, N$-Dimethylformamide & $\mathrm{DMF}$ \\
4 & Methanol & $\mathrm{MeOH}$ \\
5 & Diethyl ether & $\mathrm{Et}_{2} \mathrm{O}$ \\
\hline
\end{tabular}




\section{Photographic guide for electrochemical reaction}

Electrode electrochemical reactions were carried out using the following equipment.

- In a $10 \mathrm{~mL}$ hydrogenation tube using Ni-plate (40 mm*5 mm*0.15 mm) as anode and Pt-plate $(10 \mathrm{~mm} * 10 \mathrm{~mm} * 0.2 \mathrm{~mm})$ as cathode with the current supplied from a $36 \mathrm{~V}$ constant-current power supply purchased from Xiamen Bodong Biotechnology Ltd. (for $0.2 \mathrm{mmol}$ scale).

- In a $250 \mathrm{~mL}$ wild-mouth bottle using Ni-plate $(58 \mathrm{~mm} * 28 \mathrm{~mm} * 0.15 \mathrm{~mm})$ as anode and Pt-plate (30 mm*30 mm*0.1 mm) as cathode with the current supplied from a $36 \mathrm{~V}$ constant-current power supply purchased from Xiamen Bodong Biotechnology Ltd. (for 6.0 mmol gram-scale reaction).

\subsection{Photographic guide for first kind of equipment $(0.2 \mathrm{mmol})$}

Step 0. Overview of materials used.

From left to right: (1) Pt-plate [cathode, $10 \mathrm{~mm}$ x $10 \mathrm{~mm}$ x $0.2 \mathrm{~mm}$ ], Ni-plate was attached to a copper wire [anode, $40 \mathrm{~mm}$ x $5 \mathrm{~mm}$ x $0.15 \mathrm{~mm}$ ]; (2) a rubber stopper; (3) a $10 \mathrm{~mL}$ hydrogenation tube; (4) two hypodermic needles (the longer one for intake, the shorter for exhaust)

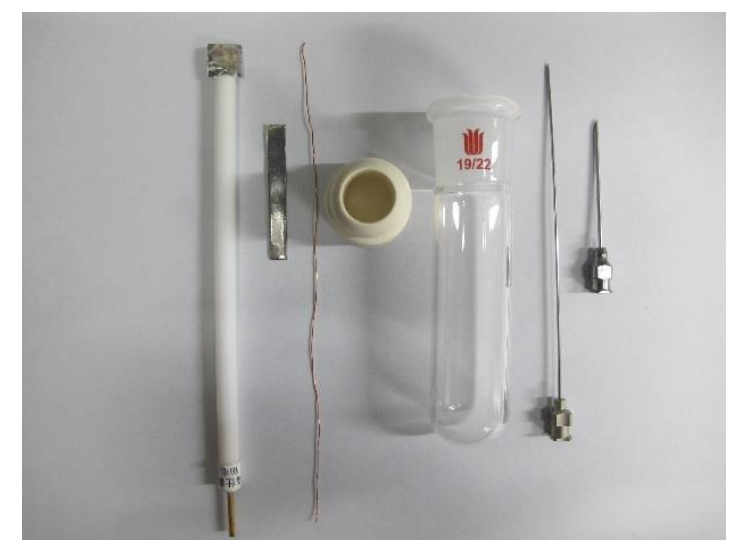

Step 1. Assembling the cell

(1) Pierce the rubber stopper with the Ni-plate and Pt-plate; (2) The stopper was fitted into the tube; (3) Pierce the rubber stopper with the two hypodermic needles (the longer one for intake, the shorter for exhaust). 


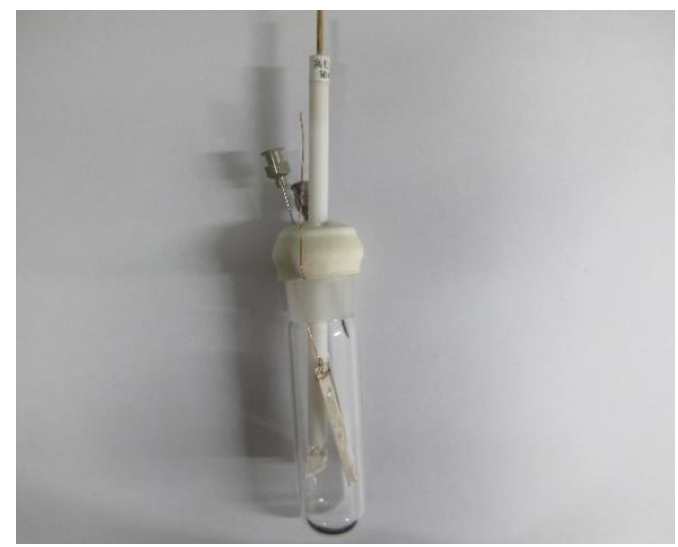

Step 2. Electrolysis

After all the materials used were added, bubbling $\mathrm{CO}_{2}$ for 10 mins and then conducting constant current electrolysis $(\mathrm{I}=8.0 \mathrm{~mA})$ using a potentiostat under continuous bubbling $\mathrm{CO}_{2}$.

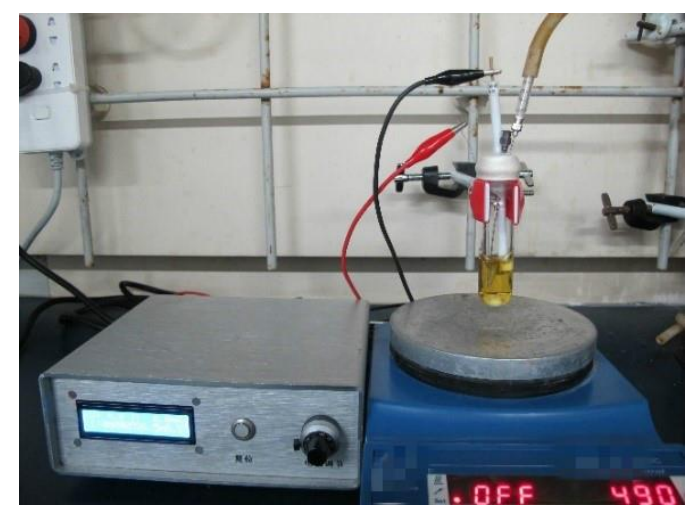

\subsection{Photographic guide for the third kind of equipment (for $6 \mathrm{mmol}$ gram-scale reaction)}

Step 0. Overview of materials used.

From left to right: (1) $250 \mathrm{~mL}$ wild-mouth bottle; (2) a rubber stopper with three holes; (3) a glass tube for intake; (4) Pt-plate [cathode, $30 \mathrm{~mm}$ x $30 \mathrm{~mm}$ x $0.1 \mathrm{~mm}$ ], Ni-plate was attached to an electrode clamp [anode, $58 \mathrm{~mm}$ x $28 \mathrm{~mm}$ x $0.15 \mathrm{~mm}$ ].

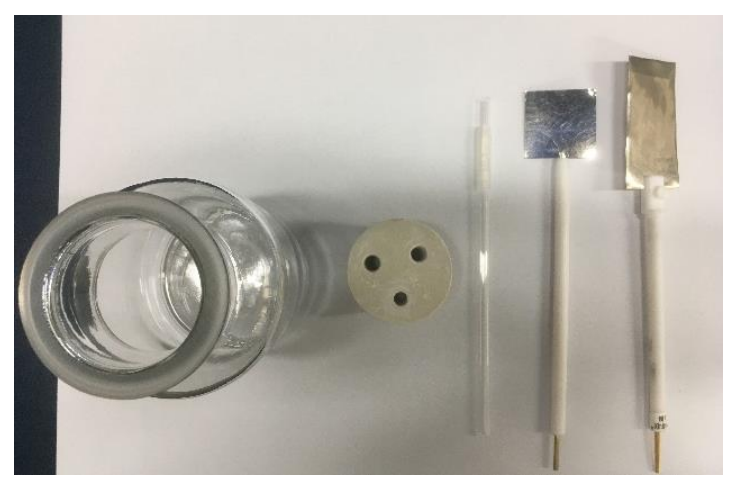


Step 1. Assembling the cell

(1) Install two electrode plates and the glass tube to the rubber stopper; (2) The rubber stopper was fitted into the bottle.
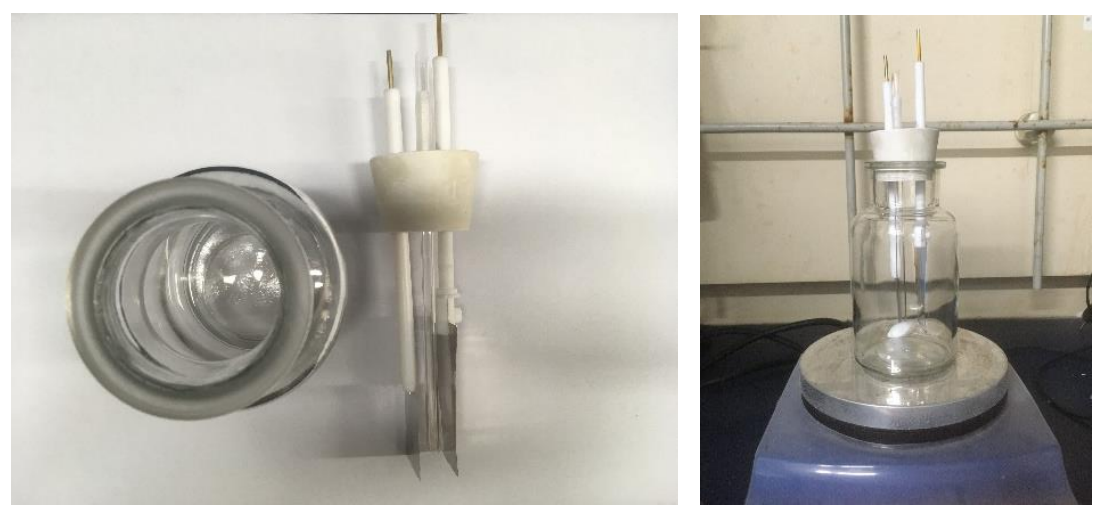

Step 2. Electrolysis

After the addition of all materials, $\mathrm{CO}_{2}$ was bubbled for 10 mins and then conducted constant current electrolysis $(\mathrm{I}=72.0 \mathrm{~mA})$ using a constant-current power supply under continuous bubbling of $\mathrm{CO}_{2}$.
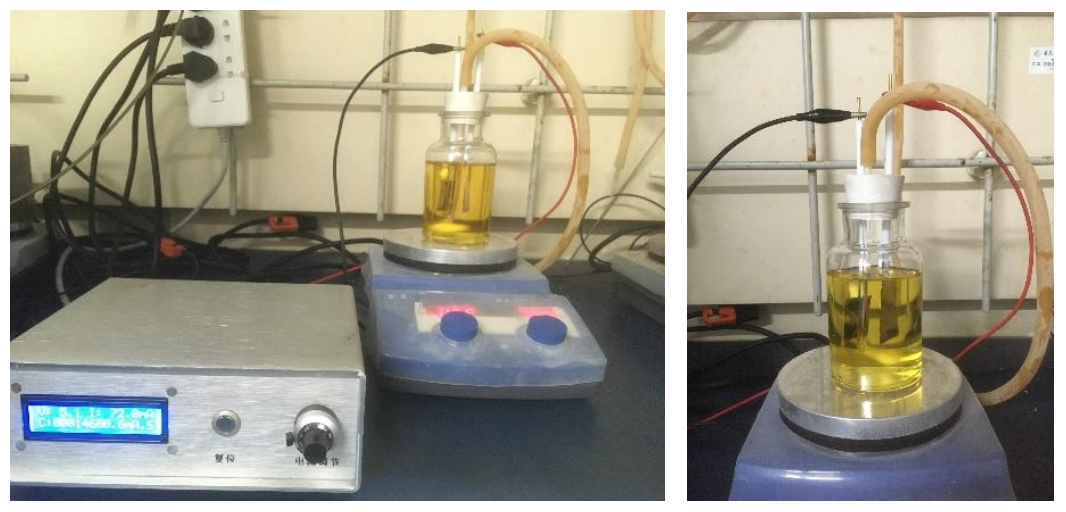


\section{Preparation and characterization of gem-difluoroalkenes}

The substrates $\mathbf{1 a - q} \mathbf{q}^{[1]}, \mathbf{1} \mathbf{r -} \mathbf{s}^{[2]}, \mathbf{1 t}^{[3]}$ were synthesized according to literature methods. Alkenes $\mathbf{1} \mathbf{m}$, 10, and $\mathbf{1 s}$ are new compounds and their characterization data is given below.

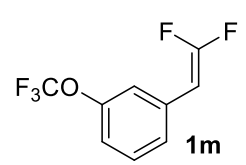

Compound 1m (colorless liquid). ${ }^{1} \mathrm{H}$ NMR $\left(400 \mathrm{MHz}, \mathrm{CDCl}_{3}\right): \delta 7.35$ (t, $J=8.0 \mathrm{~Hz}$, 1H), 7.26-7.23 (m, 1H), 7.19-7.18 (m, 1H), 7.10 (dt, $J=8.0 \mathrm{~Hz}, 1.2 \mathrm{~Hz}, 1 \mathrm{H}), 5.28$ (dd, $J=25.6 \mathrm{~Hz}, 3.2 \mathrm{~Hz}, 1 \mathrm{H}) ;{ }^{13} \mathrm{C} \mathrm{NMR}\left(100 \mathrm{MHz}, \mathrm{CDCl}_{3}\right): \delta 156.6(\mathrm{dd}, J=297.4 \mathrm{~Hz}$, $288.2 \mathrm{~Hz}, 1 \mathrm{C}$ ), 149.6, 132.5-132.4 (m, 1C), 130.0, 126.0-125.9 (m, 1C), 120.5 (q, $J=255.7 \mathrm{~Hz}, 1 \mathrm{C}$ ), 120.1-120.0 (m, 1C), 119.4, 81.5 (dd, $J=30.2 \mathrm{~Hz}, 13.3 \mathrm{~Hz}, 1 \mathrm{C}) ;{ }^{19} \mathrm{~F}$ NMR (376 MHz, $\left.\mathrm{CDCl}_{3}\right):-57.86$ (s, 3F), -80.42 (d, $J=27.1 \mathrm{~Hz}, 1 \mathrm{~F}),-82.39$ (d, $J=27.4 \mathrm{~Hz}, 1 \mathrm{~F})$; IR (ATR) v 1757.2, 1732.1, 1614.4, 1357.9, 1319.3, 1220.9, 935.5, 819.8, 742.6 cm $\mathrm{cm}^{-1}$ GC-MS (EI) m/z: $224\left(\mathrm{M}^{+}, 100\right), 158$ (7.5), 155 (8), 139 (9), 127 (40), 101 (6), 69 (11); HRMS (EI) m/z: [M] Calcd for $\mathrm{C}_{9} \mathrm{H}_{5} \mathrm{~F}_{5} \mathrm{O} 224.0261$; Found 224.0262.

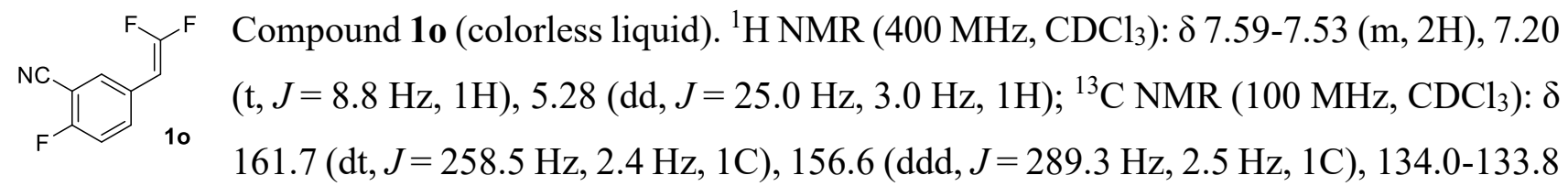

(m, 1C), 132.1-132.0 (m, 1C), 127.9-127.8 (m, 1C), 116.9 (d, $J=19.9 \mathrm{~Hz}, 1 \mathrm{C}), 113.5,102.0$ (d, $J=$ $\left.15.9 \mathrm{~Hz}, 1 \mathrm{C}), 80.2(\mathrm{dd}, J=31.2 \mathrm{~Hz}, 13.7 \mathrm{~Hz}, 1 \mathrm{C}) ;{ }^{19} \mathrm{~F} \mathrm{NMR} \mathrm{(376} \mathrm{MHz}, \mathrm{CDCl}_{3}\right): \delta-80.53(\mathrm{dd}, J=26.3$ $\mathrm{Hz}, 2.2 \mathrm{~Hz}, 1 \mathrm{~F}$ ), -81.54 (dd, $J=26.3 \mathrm{~Hz}, 4.5 \mathrm{~Hz}, 1 \mathrm{~F}$ ), -108.30 (dd, $J=4.1 \mathrm{~Hz}, 2.2 \mathrm{~Hz}, 1 \mathrm{~F}$ ); IR (ATR) v 2239.4, 1732.1, 1504.5, 1356.0, 1269.2, 1180.4, 966.3, 893.0, 839.0, $756.1 \mathrm{~cm}^{-1}$; HRMS (ESI) m/z: $[\mathrm{M}+\mathrm{Na}]^{+}$Calcd for $\mathrm{C}_{9} \mathrm{H}_{4} \mathrm{~F}_{3} \mathrm{NNa} 206.0188$; Found 206.0188.

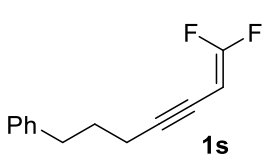

Compound 1s (colorless liquid). ${ }^{1} \mathrm{H}$ NMR (400 MHz, $\left.\mathrm{CDCl}_{3}\right): \delta$ 7.30-7.26 (m, 2H), 7.21-7.17 (m, 3H), 4.56 (dt, $J=23.2 \mathrm{~Hz}, 2.4 \mathrm{~Hz}, 1 \mathrm{H}), 2.72(\mathrm{t}, J=7.4 \mathrm{~Hz}, 2 \mathrm{H}), 2.33-$ 2.29 (m, 2H), 1.89-1.82 (m, 2H); $\left.{ }^{13} \mathrm{C} \mathrm{NMR} \mathrm{(100} \mathrm{MHz,} \mathrm{CDCl}_{3}\right): \delta 162.0(\mathrm{dd}, J=296.6$ Hz, 290.6 Hz, 1C), 141.5, 128.5, 128.4, 125.9, 93.9 (dd, $J=8.5 \mathrm{~Hz}, 3.7 \mathrm{~Hz}, 1 \mathrm{C}$ ), 69.0 (dd, $J=11.7 \mathrm{~Hz}$, $4.1 \mathrm{~Hz}, 1 \mathrm{C}), 65.4$ (dd, $J=41.4 \mathrm{~Hz}, 19.0 \mathrm{~Hz}, 1 \mathrm{C}), 34.7,30.1,18.8 ;{ }^{19} \mathrm{~F}$ NMR $\left(376 \mathrm{MHz}, \mathrm{CDCl}_{3}\right): \delta$ $77.10(\mathrm{~d}, J=10.5 \mathrm{~Hz}, 1 \mathrm{~F}),-82.35$ (d, $J=10.9 \mathrm{~Hz}, 1 \mathrm{~F})$; IR (ATR) $v$ 2358.9, 2339.6, 1759.1, 1454.3, 1240.2, 920.0, 792.7, $742.6 \mathrm{~cm}^{-1}$; GC-MS (EI) m/z: 206 (M+12), 186 (52), 178 (43), 129 (33), 128 (47), 91 (100), 77 (37), 65 (31); HRMS (EI) m/z: [M] Calcd for $\mathrm{C}_{13} \mathrm{H}_{12} \mathrm{~F}_{2}$ 206.0907; Found 206.0909.

\footnotetext{
1 Sakaguchi, H.; Uetake, Y.; Ohashi, M.; Niwa, T.; Ogoshi, S.; Hosoya, T. J. Am. Chem. Soc. 2017, 139, 12855.

2 Fujino, T.; Hinoue, T.; Usuki, Y.; Satoh, T. Org. Lett. 2016, 18, 5688.

3 Tian, H.; Xia, Q.; Wang, Q.; Dong, J.; Liu, Y.; Wang, Q. Org. Lett. 2019, 21, 4585.
} 


\section{General procedure for the electrolysis}

\subsection{General procedure for $0.2 \mathrm{mmol}$ reaction}

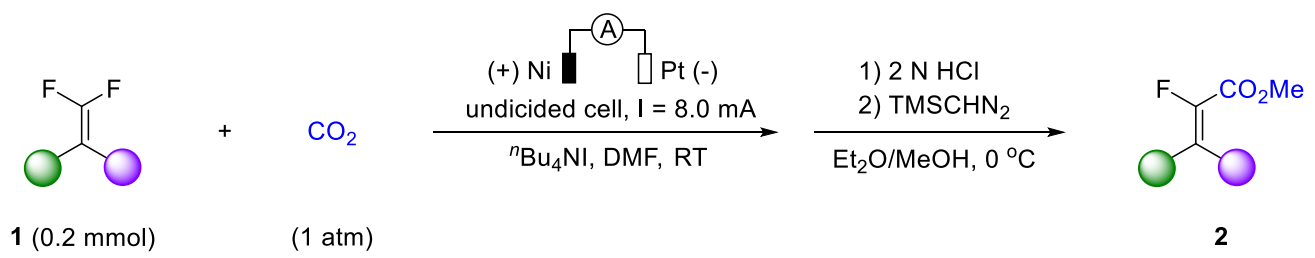

To a $10 \mathrm{~mL}$ hydrogenation tube containing a stir bar were added ${ }^{n} \mathrm{Bu} 4 \mathrm{NI}(181.0 \mathrm{mg}, 0.49 \mathrm{mmol}, 0.07$ M), DMF ( $7.0 \mathrm{~mL})$, followed by the addition of $1(0.2 \mathrm{mmol})$. Then the tube was installed with Ni-plate (40 $\mathrm{mm} \times 5 \mathrm{~mm} \times 0.15 \mathrm{~mm})$ as anode and Pt-plate $(10 \mathrm{~mm} \times 10 \mathrm{~mm} \times 0.2 \mathrm{~mm})$ as cathode. After bubbling of $\mathrm{CO}_{2}$ gas into the electrolytes for $10 \mathrm{~min}$, the reaction mixture was electrolyzed under a constant current of $8.0 \mathrm{~mA}$ until the complete consumption of the starting materials as monitored by TLC (2.5-6.5 hours). After that, the reaction mixture was transferred to a $50 \mathrm{~mL}$ erlenmeyer flask and acidized with $\mathrm{HCl}(2 \mathrm{~N}, 10 \mathrm{~mL})$. The aqueous layer extracted with EtOAc $(3 \times 10 \mathrm{~mL})$ and the combined organics were washed with $\mathrm{H}_{2} \mathrm{O}(10 \mathrm{~mL})$, brine $(10 \mathrm{~mL})$ and dried over $\mathrm{Na}_{2} \mathrm{SO}_{4}$. Part of the organics was concentrated and applied to ${ }^{19} \mathrm{~F}$ NMR analysis to determine the $Z / E$ ratio of product. Then the sample for analysis and the rest of crude residue were recombined and concentrated in vacuo. The crude residue was dissolved in $\mathrm{Et}_{2} \mathrm{O}(2.0 \mathrm{~mL})$ and $\mathrm{MeOH}(0.5 \mathrm{~mL})$ and cooled to $0{ }^{\circ} \mathrm{C}$. The hexane solution of $\mathrm{TMSCHN}_{2}(0.2 \mathrm{~mL}, 2.0 \mathrm{~mol} / \mathrm{L}, 0.4 \mathrm{mmol}, 2.0$ equivs $)$ was added at $0{ }^{\circ} \mathrm{C}$. The mixture was stirred at $0{ }^{\circ} \mathrm{C}$ for $30 \mathrm{~min}$. The volatile was removed under reduced pressure and the crude residue was purified by column chromatography using PE:EtOAc $(20: 1, \mathrm{v} / \mathrm{v})$ as the eluent to afford the desired product.

\subsection{General procedure for $6.0 \mathrm{mmol}$ gram-scale reaction.}

To a $250 \mathrm{~mL}$ wild-mouth bottle containing a stir bar were added ${ }^{n} \mathrm{Bu}_{4} \mathrm{NI}(5.43 \mathrm{~g}, 14.7 \mathrm{mmol})$ and DMF $(210 \mathrm{~mL})$, followed by the addition of $1 \mathrm{a}(1.14 \mathrm{~g}, 6.0 \mathrm{mmol})$. Then the tube was installed with Ni-plate $(58 \mathrm{~mm} \times 28 \mathrm{~mm} \times 0.15 \mathrm{~mm})$ as anode and Pt-plate $(30 \mathrm{~mm} \times 30 \mathrm{~mm} \times 0.1 \mathrm{~mm})$ as cathode. After bubbling of $\mathrm{CO}_{2}$ gas into the electrolytes for 10 mins, the reaction mixture was electrolyzed under a constant current of $72 \mathrm{~mA}$ until the complete consumption of the starting materials as monitored by TLC (20 hours). After that, the reaction mixture was transferred to a $500 \mathrm{~mL}$ round-bottom flask and then removing most of the solvent by distillation in vacuum. The residue was diluted with $50 \mathrm{mLEtOAc}$, and acidized with $\mathrm{HCl}(2 \mathrm{~N}, 40 \mathrm{~mL})$. After separation of organic layer, the aqueous layer was extracted with EtOAc $(3 \times 40 \mathrm{~mL})$ and the combined organics were washed with $\mathrm{H}_{2} \mathrm{O}(40 \mathrm{~mL})$, brine $(40 \mathrm{~mL})$ 
and dried over $\mathrm{Na}_{2} \mathrm{SO}_{4}$. Part of the organics was concentrated and applied to ${ }^{19} \mathrm{~F}$ NMR analysis revealed that the $Z / E$ ratio was 10:1. Then the sample for analysis and the rest of crude residue were recombined and concentrated in vacuo. The crude residue was dissolved in $\mathrm{Et}_{2} \mathrm{O}(60 \mathrm{~mL})$ and $\mathrm{MeOH}(15 \mathrm{~mL})$ and cooled to $0{ }^{\circ} \mathrm{C}$. The hexane solution of $\mathrm{TMSCHN}_{2}(6 \mathrm{~mL}, 2 \mathrm{~mol} / \mathrm{L}, 12 \mathrm{mmol}, 2.0$ equivs $)$ was added at $0{ }^{\circ} \mathrm{C}$. The mixture was stirred at $0{ }^{\circ} \mathrm{C}$ for $30 \mathrm{~min}$. The volatile was removed under reduced pressure and the crude residue was purified by column chromatography using PE:EtOAc $(20: 1, \mathrm{v} / \mathrm{v})$ as the eluent to afford the desired product $\mathbf{2 a}$ in $0.85 \mathrm{~g}$ with $62 \%$ yield.

\subsection{Characterization data of products}

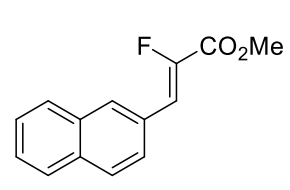

$2 \mathbf{a}$

${ }^{19} \mathrm{~F}$ NMR analysis revealed that the $Z / E$ ratio was $17: 1,(Z)-2 a$ was isolated in 32.6 $\mathrm{mg}$ (71\% yield) as white solid by column chromatography using PE:EtOAc (20:1, $\mathrm{v} / \mathrm{v})$ as the eluent; ${ }^{1} \mathrm{H} \mathrm{NMR}\left(400 \mathrm{MHz}, \mathrm{CDCl}_{3}\right): \delta 8.10(\mathrm{~s}, 1 \mathrm{H}), 7.88-7.83(\mathrm{~m}, 3 \mathrm{H})$, 7.79-7.77 (m, 1H), 7.55-7.49 (m, 2H), $7.10(\mathrm{~d}, J=35.2 \mathrm{~Hz}, 1 \mathrm{H}), 3.93(\mathrm{~s}, 3 \mathrm{H}) ;{ }^{13} \mathrm{C}$ NMR (100 MHz, $\left.\mathrm{CDCl}_{3}\right): \delta 161.9(\mathrm{~d}, J=34.2 \mathrm{~Hz}, 1 \mathrm{C}), 147.0(\mathrm{~d}, J=266.1 \mathrm{~Hz}, 1 \mathrm{C}), 133.6(\mathrm{~d}, J=2.0$ $\mathrm{Hz}, 1 \mathrm{C}), 133.2,130.8$ (d, $J=8.1 \mathrm{~Hz}, 1 \mathrm{C}), 128.7,128.6,128.5,127.7,127.3,126.8$ (d, $J=8.3 \mathrm{~Hz}, 1 \mathrm{C})$, 126.6, $118.0(\mathrm{~d}, J=4.5 \mathrm{~Hz}, 1 \mathrm{C}), 52.7 ;{ }^{19} \mathrm{~F} \mathrm{NMR}\left(376 \mathrm{MHz}, \mathrm{CDCl}_{3}\right)$ : $\delta-125.58$. The spectroscopic data correspond to those previously reported in the literature. ${ }^{[4]}$

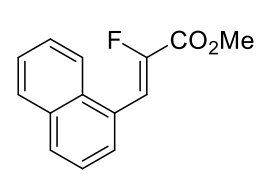

$2 \mathbf{b}$

${ }^{19} \mathrm{~F}$ NMR analysis revealed that the $Z / E$ ratio was $>20: 1,(Z)-\mathbf{2 b}$ was isolated in 34.0 mg (74\% yield) as white solid by column chromatography using PE:EtOAc (20:1, v/v) as the eluent; ${ }^{1} \mathrm{H} \mathrm{NMR}\left(400 \mathrm{MHz}, \mathrm{CDCl}_{3}\right): \delta 8.08$ (d, $\left.J=7.2 \mathrm{~Hz}, 1 \mathrm{H}\right), 7.98$ (d, $J=7.6$ $\mathrm{Hz}, 1 \mathrm{H}), 7.90-7.87(\mathrm{~m}, 2 \mathrm{H}), 7.70(\mathrm{~d}, J=33.2 \mathrm{~Hz}, 1 \mathrm{H}), 7.60-7.51(\mathrm{~m}, 3 \mathrm{H}), 3.96(\mathrm{~s}, 3 \mathrm{H})$;

${ }^{13} \mathrm{C} \mathrm{NMR}\left(100 \mathrm{MHz}, \mathrm{CDCl}_{3}\right): \delta 161.9$ (d, $\left.J=34.7 \mathrm{~Hz}, 1 \mathrm{C}\right), 147.6$ (d, $\left.J=266.5 \mathrm{~Hz}, 1 \mathrm{C}\right), 133.6,131.4$, $130.2(\mathrm{~d}, J=1.8 \mathrm{~Hz}, 1 \mathrm{C}), 128.9,128.8,127.0$ (d, $J=3.9 \mathrm{~Hz}, 1 \mathrm{C}), 126.9,126.1,125.5,123.4,114.1$ (d, $J=5.3 \mathrm{~Hz}, 1 \mathrm{C}), 52.8 ;{ }^{19} \mathrm{~F} \mathrm{NMR}\left(376 \mathrm{MHz}, \mathrm{CDCl}_{3}\right): \delta-126.14$. The spectroscopic data correspond to those previously reported in the literature. ${ }^{[4]}$

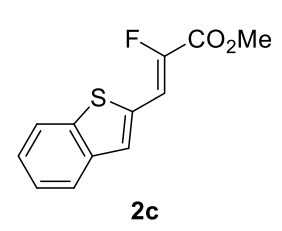

2c

${ }^{19} \mathrm{~F}$ NMR analysis revealed that the $Z / E$ ratio was $>20: 1,(Z)-2 \mathrm{c}$ was isolated in 28.2 mg (60\% yield) as yellow solid by column chromatography using PE:EtOAc (20:1, $\mathrm{v} / \mathrm{v})$ as the eluent; ${ }^{1} \mathrm{H}$ NMR (400 MHz, $\left.\mathrm{CDCl}_{3}\right): \delta$ 7.86-7.80 (m, 2H), $7.57(\mathrm{~s}, 1 \mathrm{H})$, 7.41-7.36 (m, 2H), $7.28(\mathrm{dd}, J=33.6 \mathrm{~Hz}, 0.4 \mathrm{~Hz}, 1 \mathrm{H}), 3.92(\mathrm{~s}, 3 \mathrm{H}) ;{ }^{13} \mathrm{C}$ NMR (100

\footnotetext{
${ }^{4}$ Zhu, C.; Zhang, Y.-F.; Liu, Z.-Y.; Zhou, L.; Liu, H.; Feng, C. Chem. Sci. 2019, 10, 6721.
} 
$\left.\mathrm{MHz}, \mathrm{CDCl}_{3}\right): \delta 161.3(\mathrm{~d}, J=33.0 \mathrm{~Hz}, 1 \mathrm{C}), 146.3(\mathrm{~d}, J=267.3 \mathrm{~Hz}, 1 \mathrm{C}), 141.8(\mathrm{~d}, J=7.8 \mathrm{~Hz}, 1 \mathrm{C})$, 138.8, 133.5 (d, $J=6.1 \mathrm{~Hz}, 1 \mathrm{C}), 128.3$ (d, $J=5.4 \mathrm{~Hz}, 1 \mathrm{C}), 125.9,124.8,124.3$ (d, $J=2.0 \mathrm{~Hz}, 1 \mathrm{C})$, $122.3,112.6(\mathrm{~d}, J=8.1 \mathrm{~Hz}, 1 \mathrm{C}), 52.7 ;{ }^{19} \mathrm{~F} \mathrm{NMR}\left(376 \mathrm{MHz}, \mathrm{CDCl}_{3}\right): \delta-122.59$. The spectroscopic data correspond to those previously reported in the literature. ${ }^{[4]}$

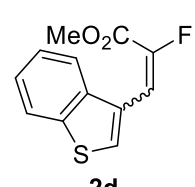

${ }^{19} \mathrm{~F}$ NMR analysis revealed that the $Z / E$ ratio was $1: 2,(E)-\mathbf{2 d}$ was isolated in $21.1 \mathrm{mg}(45 \%$ yield) as white solid by column chromatography using PE:EtOAc $(20: 1, \mathrm{v} / \mathrm{v})$ as the eluent; $\mathrm{Mp} 66-68{ }^{\circ} \mathrm{C} ;{ }^{1} \mathrm{H} \mathrm{NMR}\left(400 \mathrm{MHz}, \mathrm{CDCl}_{3}\right): \delta 8.32(\mathrm{~s}, 1 \mathrm{H}), 7.89-7.87(\mathrm{~m}, 1 \mathrm{H}), 7.76-7.73$ $(\mathrm{m}, 1 \mathrm{H}), 7.46-7.37(\mathrm{~m}, 2 \mathrm{H}), 7.12(\mathrm{dd}, J=23.0 \mathrm{~Hz}, 1.0 \mathrm{~Hz}, 1 \mathrm{H}), 3.84(\mathrm{~s}, 3 \mathrm{H}) ;{ }^{13} \mathrm{C}$ NMR $(100 \mathrm{MHz}$, $\left.\mathrm{CDCl}_{3}\right): \delta 161.1(\mathrm{~d}, J=34.8 \mathrm{~Hz}, 1 \mathrm{C}), 147.6(\mathrm{~d}, J=255.3 \mathrm{~Hz}, 1 \mathrm{C}), 139.3,138.6(\mathrm{~d}, J=2.5 \mathrm{~Hz}, 1 \mathrm{C})$, 129.7 (d, $J=4.3 \mathrm{~Hz}, 1 \mathrm{C}), 124.7$ (d, $J=9.4 \mathrm{~Hz}, 1 \mathrm{C}), 124.6,124.6,122.9,121.2,113.8$ (d, $J=28.7 \mathrm{~Hz}$, 1C), 52.4; ${ }^{19} \mathrm{~F}$ NMR (376 MHz, $\left.\mathrm{CDCl}_{3}\right): \delta$-117.39; IR (ATR) v 2360.9, 2341.6, 1383.0, 1323.2, 1267.2, 1230.6, 1130.3, 1047.4, 740.7, $669.3 \mathrm{~cm}^{-1}$; HRMS (ESI) m/z: $[\mathrm{M}+\mathrm{Na}]^{+}$Calcd for $\mathrm{C}_{12} \mathrm{H}_{9} \mathrm{FNaO}_{2} \mathrm{~S}$ 259.0199; Found 259.0203.

$Z$-isomer was further isolated in $10.2 \mathrm{mg}$ ( $22 \%$ yield) as white solid by column chromatography using PE:EtOAc (20:1, v/v) as the eluent; $\mathrm{Mp} \mathrm{80-81}{ }^{\circ} \mathrm{C} ;{ }^{1} \mathrm{H}$ NMR $\left(400 \mathrm{MHz}, \mathrm{CDCl}_{3}\right): \delta 8.12(\mathrm{~s}, 1 \mathrm{H})$, 7.92-7.89 (m, 2H), 7.50-7.40 (m, 2H), 7.32 (d, $J=34.4 \mathrm{~Hz}, 1 \mathrm{H}), 3.94$ (s, 3H); ${ }^{13} \mathrm{C}$ NMR (100 MHz, $\left.\mathrm{CDCl}_{3}\right): \delta 161.7(\mathrm{~d}, J=33.8 \mathrm{~Hz}, 1 \mathrm{C}), 147.8(\mathrm{~d}, J=265.3 \mathrm{~Hz}, 1 \mathrm{C}), 139.2,137.8,130.7$ (d, $J=15.6 \mathrm{~Hz}$, 1C), $125.8(\mathrm{~d}, J=3.8 \mathrm{~Hz}, 1 \mathrm{C}), 125.0,124.8,122.8,121.2,109.0$ (d, $J=7.2 \mathrm{~Hz}, 1 \mathrm{C}), 52.7$; ${ }^{19} \mathrm{~F}$ NMR $\left(376 \mathrm{MHz}, \mathrm{CDCl}_{3}\right): \delta$-123.03; IR (ATR) v 2341.6, 1732.1, 1438.9, 1334.7, 1271.1, 1246.0, 1105.2, 1047.4, 895.0, $738.7 \mathrm{~cm}^{-1}$; HRMS (ESI) m/z: $[\mathrm{M}+\mathrm{Na}]^{+}$Calcd for $\mathrm{C}_{12} \mathrm{H}_{9} \mathrm{FNaO}_{2} \mathrm{~S}$ 259.0199; Found 259.0190 .

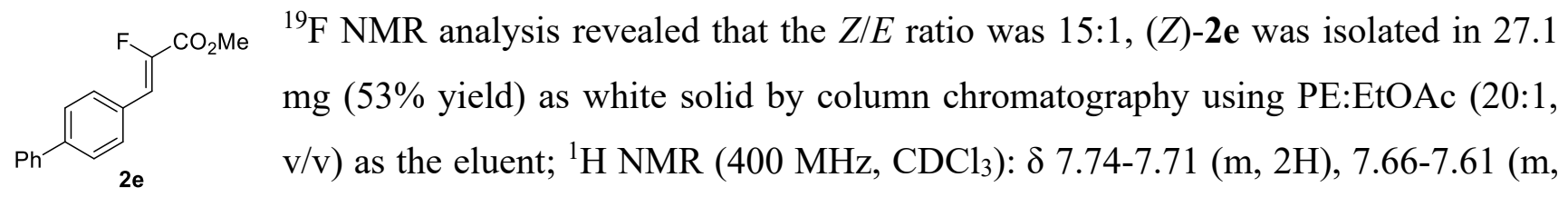
4H), 7.48-7.44 (m, 2H), 7.40-7.35 (m, 1H), $6.97(\mathrm{~d}, J=8.8 \mathrm{~Hz}, 1 \mathrm{H}), 3.91(\mathrm{~s}, 3 \mathrm{H}) ;{ }^{13} \mathrm{C} \mathrm{NMR}(100 \mathrm{MHz}$, $\left.\mathrm{CDCl}_{3}\right): \delta 161.9(\mathrm{~d}, J=34.1 \mathrm{~Hz}, 1 \mathrm{C}), 146.9(\mathrm{~d}, J=265.7 \mathrm{~Hz}, 1 \mathrm{C}), 142.4(\mathrm{~d}, J=2.9 \mathrm{~Hz}, 1 \mathrm{C}), 140.1$, $130.8(\mathrm{~d}, J=8.1 \mathrm{~Hz}, 1 \mathrm{C}), 130.0$ (d, $J=4.5 \mathrm{~Hz}, 1 \mathrm{C}), 128.9,127.9,127.4,127.1,117.5$ (d, $J=4.6 \mathrm{~Hz}$, 1C), 52.7; ${ }^{19} \mathrm{~F}$ NMR (376 $\left.\mathrm{MHz}, \mathrm{CDCl}_{3}\right): \delta-125.48$. The spectroscopic data correspond to those previously reported in the literature. ${ }^{[4]}$ 


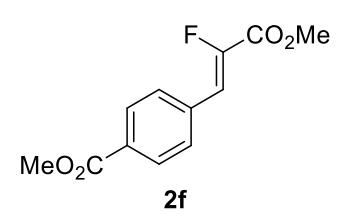

${ }^{19} \mathrm{~F}$ NMR analysis revealed that the $Z / E$ ratio was $>20: 1,(Z)-\mathbf{2 f}$ was isolated in $36.1 \mathrm{mg}$ (76\% yield) as white solid by column chromatography using PE:EtOAc $(7: 1, \mathrm{v} / \mathrm{v})$ as the eluent; ${ }^{1} \mathrm{H}$ NMR $\left(400 \mathrm{MHz}, \mathrm{CDCl}_{3}\right): \delta 8.06(\mathrm{dd}, J=6.6 \mathrm{~Hz}, 1.8$ $\mathrm{Hz}, 2 \mathrm{H}), 7.70$ (dd, $J=6.4 \mathrm{~Hz}, 2.0 \mathrm{~Hz}, 1 \mathrm{H}), 6.96$ (d, $J=34.4 \mathrm{~Hz}, 1 \mathrm{H}), 3.94$ (s, 3H), $3.92(\mathrm{~s}, 3 \mathrm{H}) ;{ }^{13} \mathrm{C} \mathrm{NMR}\left(100 \mathrm{MHz}, \mathrm{CDCl}_{3}\right): \delta 166.4,161.4(\mathrm{~d}, J=34.4 \mathrm{~Hz}, 1 \mathrm{C}), 147.9(\mathrm{~d}, J=269.9 \mathrm{~Hz}$, 1C), 135.3 (d, $J=4.5 \mathrm{~Hz}, 1 \mathrm{C}), 130.8$ (d, $J=2.8 \mathrm{~Hz}, 1 \mathrm{C}), 130.1$ (d, $J=8.3 \mathrm{~Hz}, 1 \mathrm{C}), 129.9,116.5$ (d, $J=$ $4.4 \mathrm{~Hz}, 1 \mathrm{C}), 52.8,52.3 ;{ }^{19} \mathrm{~F} \mathrm{NMR}\left(376 \mathrm{MHz}, \mathrm{CDCl}_{3}\right): \delta-122.24$. The spectroscopic data correspond to those previously reported in the literature. ${ }^{[5]}$

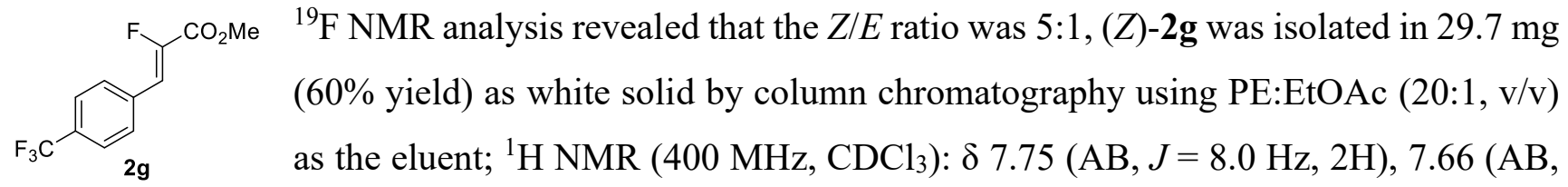
$J=8.4 \mathrm{~Hz}, 2 \mathrm{H}), 6.96(\mathrm{~d}, J=34.4 \mathrm{~Hz}, 1 \mathrm{H}), 3.92(\mathrm{~s}, 3 \mathrm{H}) ;{ }^{13} \mathrm{C} \mathrm{NMR}\left(100 \mathrm{MHz}, \mathrm{CDCl}_{3}\right): \delta 161.4(\mathrm{~d}, J=$ $34.3 \mathrm{~Hz}, 1 \mathrm{C}), 148.0$ (d, $J=269.9 \mathrm{~Hz}, 1 \mathrm{C}), 134.4$ (d, $J=4.3 \mathrm{~Hz}, 1 \mathrm{C}), 131.2$ (dd, $J=32.6 \mathrm{~Hz}, 3.0 \mathrm{~Hz}$, 1C), 130.4 (d, $J=8.3 \mathrm{~Hz}, 1 \mathrm{C}), 125.7$ (q, $J=3.7 \mathrm{~Hz}, 1 \mathrm{C}), 123.8$ (q, $J=270.6 \mathrm{~Hz}, 1 \mathrm{C}), 116.1$ (d, $J=4.4$ $\mathrm{Hz}, 1 \mathrm{C}), 52.8 ;{ }^{19} \mathrm{~F}$ NMR $\left(376 \mathrm{MHz}, \mathrm{CDCl}_{3}\right): \delta-62.98(\mathrm{~s}, 3 \mathrm{~F}),-122.36(\mathrm{~s}, 1 \mathrm{~F})$. The spectroscopic data correspond to those previously reported in the literature. ${ }^{[4]}$

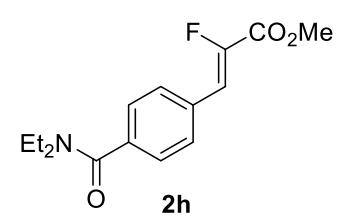

${ }^{19} \mathrm{~F}$ NMR analysis revealed that the $Z / E$ ratio was $>20: 1,(Z)-\mathbf{2 h}$ was isolated in $39.1 \mathrm{mg}$ (70\% yield) as white solid by column chromatography using PE:EtOAc $(2: 1, \mathrm{v} / \mathrm{v})$ as the eluent; ${ }^{1} \mathrm{H} \mathrm{NMR}\left(400 \mathrm{MHz}, \mathrm{CDCl}_{3}\right): \delta 7.67(\mathrm{AB}, J=8.4 \mathrm{~Hz}, 2 \mathrm{H})$, $7.41(\mathrm{AB}, J=8.4 \mathrm{~Hz}, 2 \mathrm{H}), 6.93(\mathrm{~d}, J=34.8 \mathrm{~Hz}, 1 \mathrm{H}), 3.91(\mathrm{~s}, 3 \mathrm{H}), 3.55(\mathrm{~s}, 2 \mathrm{H})$, $3.26(\mathrm{~s}, 2 \mathrm{H}), 1.25(\mathrm{~s}, 3 \mathrm{H}), 1.13(\mathrm{~s}, 3 \mathrm{H}) ;{ }^{13} \mathrm{C} \mathrm{NMR}\left(100 \mathrm{MHz}, \mathrm{CDCl}_{3}\right): \delta 170.4,161.6(\mathrm{~d}, J=34.3 \mathrm{~Hz}$, 1C), 147.3 (d, $J=267.4 \mathrm{~Hz}, 1 \mathrm{C}), 138.4$ (d, $J=2.8 \mathrm{~Hz}, 1 \mathrm{C}), 131.8$ (d, $J=4.4 \mathrm{~Hz}, 1 \mathrm{C}), 130.3$ (d, $J=8.2$ $\mathrm{Hz}, 1 \mathrm{C}), 126.8,116.9$ (d, $J=4.5 \mathrm{~Hz}, 1 \mathrm{C}), 52.7,43.3,39.3,14.2,12.9 ;{ }^{19} \mathrm{~F} \mathrm{NMR}\left(376 \mathrm{MHz}, \mathrm{CDCl}_{3}\right): \delta$ -124.21. The spectroscopic data correspond to those previously reported in the literature. ${ }^{[4]}$

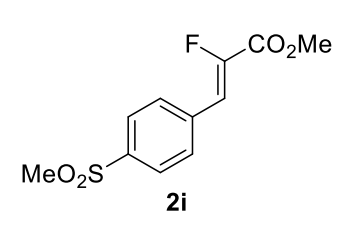

${ }^{19} \mathrm{~F}$ NMR analysis revealed that the $Z / E$ ratio was $>20: 1,(Z)-2 \mathbf{i}$ was isolated in $27.3 \mathrm{mg}$ (53\% yield) as yellow solid by column chromatography using PE:EtOAc $(1: 1, \mathrm{v} / \mathrm{v})$ as the eluent; ${ }^{1} \mathrm{H} \mathrm{NMR}\left(400 \mathrm{MHz}, \mathrm{CDCl}_{3}\right): \delta 7.98(\mathrm{ABd}, J=6.6 \mathrm{~Hz}, 1.8$ $\mathrm{Hz}, 2 \mathrm{H}), 7.82(\mathrm{ABd}, J=6.6 \mathrm{~Hz}, 1.8 \mathrm{~Hz}, 2 \mathrm{H}), 6.98(\mathrm{~d}, J=33.6 \mathrm{~Hz}, 1 \mathrm{H}), 3.93$ (s,

\footnotetext{
5 Yan, S.-S.; Wu, D.-S.; Ye, J.-H.; Gong, L.; Zeng, X.; Ran, C.-K.; Gui, Y.-Y.; Li, J.; Yu, D.-G. ACS Catal. $2019,9,6987$.
} 
3H), 3.07 (s, 3H); ${ }^{13} \mathrm{C} \mathrm{NMR}\left(100 \mathrm{MHz}, \mathrm{CDCl}_{3}\right): \delta 161.1(\mathrm{~d}, J=34.3 \mathrm{~Hz}, 1 \mathrm{C}), 148.6(\mathrm{~d}, J=272.0 \mathrm{~Hz}$, 1C), 141.0 (d, $J=2.9 \mathrm{~Hz}, 1 \mathrm{C}), 136.2$ (d, $J=4.3 \mathrm{~Hz}, 1 \mathrm{C}), 130.8$ (d, $J=8.2 \mathrm{~Hz}, 1 \mathrm{C}), 127.8,115.5$ (d, $J=$ 4.3 Hz, 1C), 53.0, 44.4; ${ }^{19} \mathrm{~F}$ NMR (376 $\left.\mathrm{MHz} \mathrm{CDCl}_{3}\right): \delta-120.52$. The spectroscopic data correspond to those previously reported in the literature. ${ }^{[4]}$

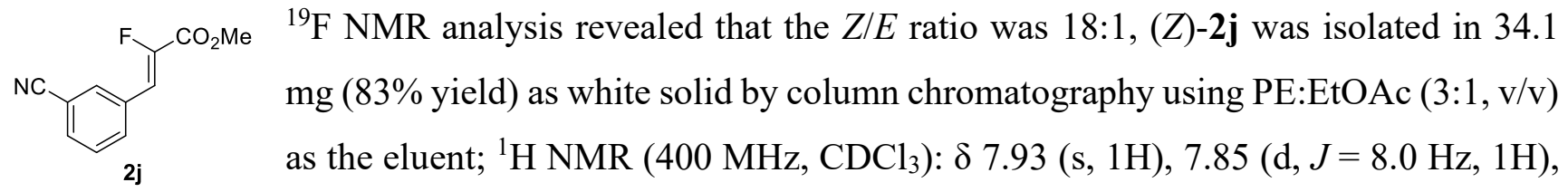
7.67-7.64 (m, 1H), 7.53 (t, $J=7.8 \mathrm{~Hz}, 1 \mathrm{H}), 6.91(\mathrm{~d}, J=33.6 \mathrm{~Hz}, 1 \mathrm{H}), 3.92(\mathrm{~s}, 3 \mathrm{H}) ;{ }^{13} \mathrm{C} \mathrm{NMR}(100 \mathrm{MHz}$, $\left.\mathrm{CDCl}_{3}\right): \delta 161.1(\mathrm{~d}, J=34.1 \mathrm{~Hz}, 1 \mathrm{C}), 148.2(\mathrm{~d}, J=270.4 \mathrm{~Hz}, 1 \mathrm{C}), 134.1(\mathrm{~d}, J=8.0 \mathrm{~Hz}, 1 \mathrm{C}), 133.4(\mathrm{~d}$, $J=8.4 \mathrm{~Hz}, 1 \mathrm{C}), 132.7$ (d, $J=2.5 \mathrm{~Hz}, 1 \mathrm{C}), 132.3$ (d, $J=4.1 \mathrm{~Hz}, 1 \mathrm{C}), 129.7,118.1,115.3$ (d, $J=4.4 \mathrm{~Hz}$, 1C), 113.3, 52.9; ${ }^{19} \mathrm{~F}$ NMR $\left(376 \mathrm{MHz}, \mathrm{CDCl}_{3}\right): \delta-121.85$. The spectroscopic data correspond to those previously reported in the literature. ${ }^{[5]}$

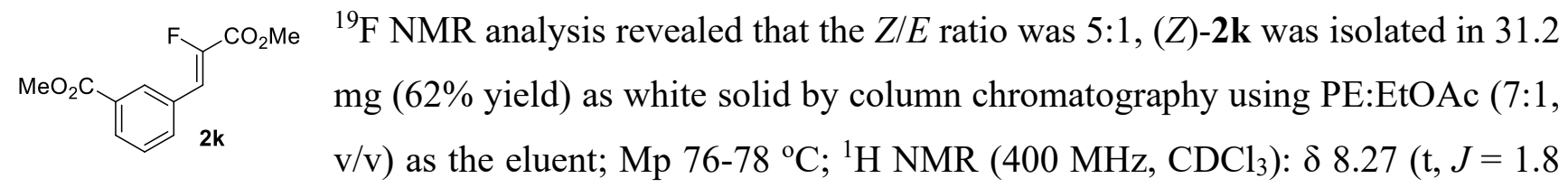
$\mathrm{Hz}, 1 \mathrm{H}), 8.06-8.03(\mathrm{~m}, 1 \mathrm{H}), 7.87(\mathrm{~d}, J=8.0 \mathrm{~Hz}, 1 \mathrm{H}), 7.50$ (t, $J=7.8 \mathrm{~Hz}, 1 \mathrm{H}), 6.97(\mathrm{~d}, J=34.8 \mathrm{~Hz}, 1 \mathrm{H})$, $3.94(\mathrm{~s}, 3 \mathrm{H}), 3.91(\mathrm{~s}, 3 \mathrm{H}) ;{ }^{13} \mathrm{C} \mathrm{NMR}\left(100 \mathrm{MHz}, \mathrm{CDCl}_{3}\right): \delta 166.4,161.5(\mathrm{~d}, J=34.1 \mathrm{~Hz}, 1 \mathrm{C}), 147.4(\mathrm{~d}$, $J=267.8 \mathrm{~Hz}, 1 \mathrm{C}), 134.2(\mathrm{~d}, J=9.0 \mathrm{~Hz}, 1 \mathrm{C}), 131.4,131.3,130.8,130.6$ (d, $J=2.6 \mathrm{~Hz}, 1 \mathrm{C}), 129.0,116.7$ $(\mathrm{d}, J=4.5 \mathrm{~Hz}, 1 \mathrm{C}), 52.8,52.3 ;{ }^{19} \mathrm{~F}$ NMR (376 MHz, $\left.\mathrm{CDCl}_{3}\right): \delta-123.91$; IR (ATR) v 1732.1, 1440.8, 1346.3, 1290.4, 1190.1, 1114.9, 1095.6, 968.3, 850.6, $761.9 \mathrm{~cm}^{-1} ; \mathrm{HRMS}(\mathrm{ESI}) \mathrm{m} / \mathrm{z}:[\mathrm{M}+\mathrm{Na}]^{+}$Calcd for $\mathrm{C}_{12} \mathrm{H}_{11} \mathrm{FNaO}_{4} 261.0534$; Found 261.0531.

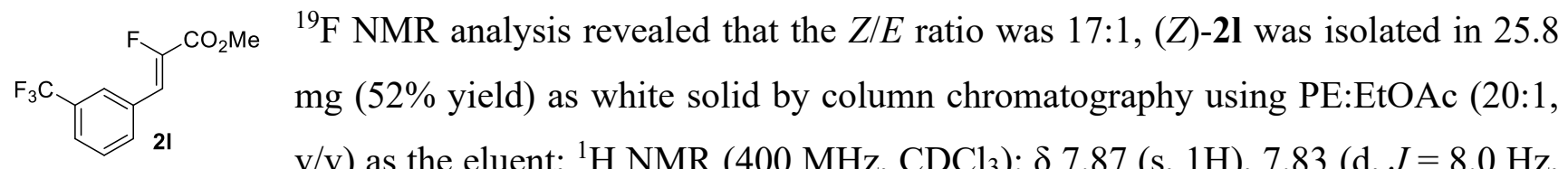
$1 \mathrm{H}), 7.63(\mathrm{~d}, J=8.0 \mathrm{~Hz}, 1 \mathrm{H}), 7.54(\mathrm{t}, J=7.8 \mathrm{~Hz}, 1 \mathrm{H}), 6.96(\mathrm{~d}, J=34.0 \mathrm{~Hz}, 1 \mathrm{H}), 3.92(\mathrm{~s}, 3 \mathrm{H}) ;{ }^{13} \mathrm{C} \mathrm{NMR}$ (100 MHz, $\left.\mathrm{CDCl}_{3}\right): \delta 161.4(\mathrm{~d}, J=34.3 \mathrm{~Hz}, 1 \mathrm{C}), 147.7$ (d, $\left.J=269.0 \mathrm{~Hz}, 1 \mathrm{C}\right), 133.2(\mathrm{~d}, J=8.4 \mathrm{~Hz}, 1 \mathrm{C})$, $131.8(\mathrm{~d}, J=4.3 \mathrm{~Hz}, 1 \mathrm{C}), 131.4$ (q, $J=32.3 \mathrm{~Hz}, 1 \mathrm{C}), 129.4,126.9-126.7$ (m, 1C), 126.2-126.1 (m, 1C), 123.7 (q, $J=270.7 \mathrm{~Hz}, 1 \mathrm{C}), 116.1(\mathrm{~d}, J=4.4 \mathrm{~Hz}, 1 \mathrm{C}), 52.8 ;{ }^{19} \mathrm{~F} \mathrm{NMR}\left(376 \mathrm{MHz}, \mathrm{CDCl}_{3}\right): \delta-62.97(\mathrm{~s}$, $3 \mathrm{~F}),-123.03(\mathrm{~s}, 1 \mathrm{~F})$. The spectroscopic data correspond to those previously reported in the literature. ${ }^{[4]}$ 


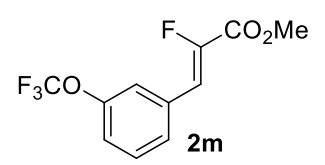

${ }^{19} \mathrm{~F}$ NMR analysis revealed that the $Z / E$ ratio was 8:1, (Z)-2m was isolated in 27.4 $\mathrm{mg}(52 \%$ yield) as light yellow liquid by column chromatography using PE:EtOAc (20:1, v/v) as the eluent; ${ }^{1} \mathrm{H}$ NMR $\left(400 \mathrm{MHz} \mathrm{CDCl}_{3}\right): \delta 7.55(\mathrm{~d}, J=8.0 \mathrm{~Hz}, 1 \mathrm{H})$, $7.51(\mathrm{~s}, 1 \mathrm{H}), 7.44(\mathrm{t}, J=8.0 \mathrm{~Hz}, 1 \mathrm{H}), 7.26-7.23(\mathrm{~m}, 1 \mathrm{H}), 6.91(\mathrm{~d}, J=34.0 \mathrm{~Hz}, 1 \mathrm{H}), 3.91(\mathrm{~s}, 3 \mathrm{H}) ;{ }^{13} \mathrm{C}$ NMR (100 MHz, $\left.\mathrm{CDCl}_{3}\right): \delta 161.4(\mathrm{~d}, J=34.2 \mathrm{~Hz}, 1 \mathrm{C}), 149.5,147.6(\mathrm{~d}, J=269.0 \mathrm{~Hz}, 1 \mathrm{C}), 132.9(\mathrm{~d}, J$ $=4.3 \mathrm{~Hz}, 1 \mathrm{C}), 130.2,128.5(\mathrm{~d}, J=7.8 \mathrm{~Hz}, 1 \mathrm{C}), 122.4$ (d, $J=8.8 \mathrm{~Hz}, 1 \mathrm{C}), 122.0,120.4(\mathrm{q}, J=256.0$ $\mathrm{Hz}, 1 \mathrm{C}), 116.2$ (d, $J=4.4 \mathrm{~Hz}, 1 \mathrm{C}), 52.8 ;{ }^{19} \mathrm{~F} \mathrm{NMR}\left(376 \mathrm{MHz}, \mathrm{CDCl}_{3}\right):-57.85$ (s, 3F), -123.05 (s, 1F); IR $($ ATR $) \vee 2987.7,2362.8,1487.1,1356.0,1303.9,1267.2,1159.2,1066.6,788.9,740.7 \mathrm{~cm}^{-1}$; HRMS (ESI) $\mathrm{m} / \mathrm{z}$ : $[\mathrm{M}+\mathrm{Na}]^{+}$Calcd for $\mathrm{C}_{11} \mathrm{H}_{8} \mathrm{~F}_{4} \mathrm{NaO}_{3}$ 287.0302; Found 287.0293.

${ }^{19} \mathrm{~F} \mathrm{NMR}$ analysis revealed that the $Z / E$ ratio was $10: 1,(Z)-2 \mathrm{n}$ was isolated in $23.8 \mathrm{mg}$
$(48 \%$ yield $)$ as white solid by column chromatography using PE:EtOAc $(20: 1, \mathrm{v} / \mathrm{v})$ as 1H), 7.60 (t, $J=7.6 \mathrm{~Hz}, 1 \mathrm{H}), 7.47$ (t, $J=7.6 \mathrm{~Hz}, 1 \mathrm{H}), 7.27$ (dd, $J=32.2 \mathrm{~Hz}, 1.8 \mathrm{~Hz}, 1 \mathrm{H}), 3.92(\mathrm{~s}, 3 \mathrm{H})$; ${ }^{13} \mathrm{C} \mathrm{NMR}\left(100 \mathrm{MHz}, \mathrm{CDCl}_{3}\right): \delta 161.2(\mathrm{~d}, J=35.0 \mathrm{~Hz}, 1 \mathrm{C}), 147.8(\mathrm{~d}, J=268.1 \mathrm{~Hz}, 1 \mathrm{C}), 132.0,131.5$, 131.4, 129.1 (d, $J=1.7 \mathrm{~Hz}, 1 \mathrm{C}), 129.0-128.4$ (m, 1C), 126.1 (q, $J=5.6 \mathrm{~Hz}, 1 \mathrm{C}), 123.8$ (q, $J=272.2$ $\mathrm{Hz}, 1 \mathrm{C}), 112.8(\mathrm{q}, J=2.1 \mathrm{~Hz}, 1 \mathrm{C}), 52.9(\mathrm{~s}, 1 \mathrm{C}) ;{ }^{19} \mathrm{~F} \mathrm{NMR}\left(376 \mathrm{MHz}, \mathrm{CDCl}_{3}\right): \delta-59.56(\mathrm{~d}, J=2.3 \mathrm{~Hz}$ $3 \mathrm{~F}),-124.08(\mathrm{~d}, J=2.3 \mathrm{~Hz}, 1 \mathrm{~F})$. The spectroscopic data correspond to those previously reported in the literature. ${ }^{[4]}$

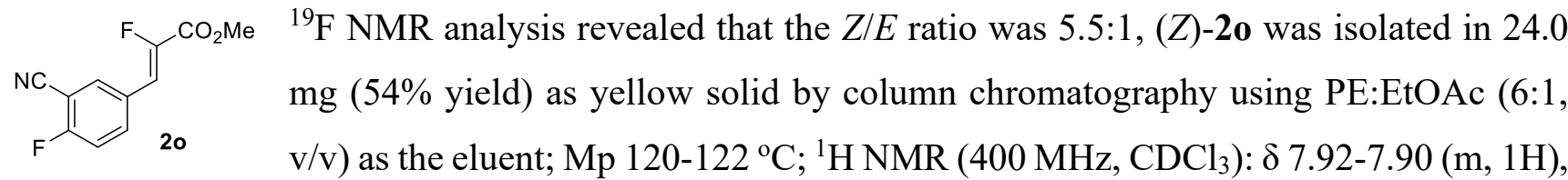
7.89-7.85 (m, 1H), 7.28 (t, $J=8.6 \mathrm{~Hz}, 1 \mathrm{H}), 6.87(\mathrm{~d}, J=33.6 \mathrm{~Hz}, 1 \mathrm{H}), 3.92(\mathrm{~s}, 3 \mathrm{H}) ;{ }^{13} \mathrm{C} \mathrm{NMR}(100 \mathrm{MHz}$, $\left.\mathrm{CDCl}_{3}\right): \delta 163.0(\mathrm{dd}, J=262.1 \mathrm{~Hz}, 3.6 \mathrm{~Hz}, 1 \mathrm{C}), 161.0(\mathrm{~d}, J=34.1 \mathrm{~Hz}, 1 \mathrm{C}), 147.9$ (dd, $J=269.8 \mathrm{~Hz}$, $2.5 \mathrm{~Hz}, 1 \mathrm{C}), 136.5$ (t, $J=8.4 \mathrm{~Hz}, 1 \mathrm{C}), 135.0$ (d, $J=9.1 \mathrm{~Hz}, 1 \mathrm{C}), 128.4$ (t, $J=4.1 \mathrm{~Hz}, 1 \mathrm{C}), 117.2$ (d, $J=$ $19.8 \mathrm{~Hz}, 1 \mathrm{C}), 114.2$ (dd, $J=4.5 \mathrm{~Hz}, 1.2 \mathrm{~Hz}, 1 \mathrm{C}), 113.2,102.5$ (d, $J=16.0 \mathrm{~Hz}, 1 \mathrm{C}), 53.0 ;{ }^{19} \mathrm{~F}$ NMR $(376$ $\mathrm{MHz}, \mathrm{CDCl}_{3}$ ): $\delta-103.86(\mathrm{~d}, J=2.6 \mathrm{~Hz}, 1 \mathrm{~F}),-122.98$ (s, 1F); IR (ATR) v 1734.0, 1504.5, 1437.0, 1352.1, 1217.1, 1101.4, 966.3, 912.3, 833.3, $761.9 \mathrm{~cm}^{-1}$; HRMS (ESI) m/z: $[\mathrm{M}+\mathrm{Na}]^{+}$Calcd for $\mathrm{C}_{11} \mathrm{H}_{7} \mathrm{~F}_{2} \mathrm{NNaO}_{2}$ 246.0337; Found 246.0334. 


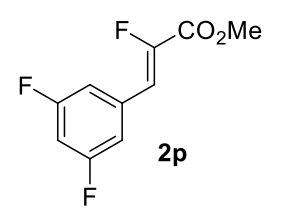

${ }^{19} \mathrm{~F}$ NMR analysis revealed that the $Z / E$ ratio was $>20: 1,(Z)-2 \mathbf{p}$ was isolated in 19.3 $\mathrm{mg}(45 \%$ yield) as white solid by column chromatography using PE:EtOAc $(15: 1, \mathrm{v} / \mathrm{v})$ as the eluent; ${ }^{1} \mathrm{H}$ NMR (400 MHz, $\left.\mathrm{CDCl}_{3}\right): \delta 7.19-7.14(\mathrm{~m}, 2 \mathrm{H}), 6.84(\mathrm{~d}, J=33.2 \mathrm{~Hz}$, $1 \mathrm{H}), 6.84(\mathrm{tt}, J=8.8 \mathrm{~Hz}, 2.4 \mathrm{~Hz}, 1 \mathrm{H}), 3.91(\mathrm{~s}, 3 \mathrm{H}) ;{ }^{13} \mathrm{C} \mathrm{NMR}\left(125 \mathrm{MHz}, \mathrm{CDCl}_{3}\right): \delta 163.0$ (dd, $J=246.9$, 12.6 Hz, 1C), 161.2 (d, $J=34.1 \mathrm{~Hz}, 1 \mathrm{C}), 148.0$ (d, $J=270.5 \mathrm{~Hz}, 1 \mathrm{C}), 133.7$ (td, $J=10.3,4.0 \mathrm{~Hz}, 1 \mathrm{C})$, $115.6(\mathrm{q}, J=3.4 \mathrm{~Hz}, 1 \mathrm{C}), 113.1-112.8(\mathrm{~m}, 1 \mathrm{C}), 105.2(\mathrm{td}, J=25.3,2.3 \mathrm{~Hz}, 1 \mathrm{C}), 52.9 ;{ }^{19} \mathrm{~F}$ NMR $(376$ $\mathrm{MHz}, \mathrm{CDCl}_{3}$ ): $\delta-109.02(\mathrm{~d}, J=1.9 \mathrm{~Hz}, 2 \mathrm{~F}),-121.50(\mathrm{~s}, 1 \mathrm{~F})$. The spectroscopic data correspond to those previously reported in the literature. ${ }^{[4]}$

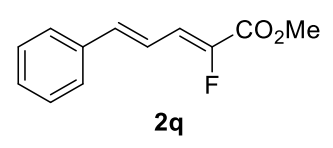

${ }^{19} \mathrm{~F}$ NMR analysis revealed that the $Z / E$ ratio was 5:1, (Z)-2q was isolated in 14.3 $\mathrm{mg}$ (35\% yield) as white solid by column chromatography using PE:EtOAc (10:1, $\mathrm{v} / \mathrm{v}$ ) as the eluent; ${ }^{1} \mathrm{H}$ NMR (400 MHz, $\left.\mathrm{CDCl}_{3}\right): \delta$ 7.50-7.48 (m, $\left.2 \mathrm{H}\right), 7.39-7.29(\mathrm{~m}$, $3 \mathrm{H}), 7.12-7.05(\mathrm{~m}, 1 \mathrm{H}), 6.88-6.74(\mathrm{~m}, 2 \mathrm{H}), 3.87(\mathrm{~s}, 3 \mathrm{H}) ;{ }^{13} \mathrm{C} \mathrm{NMR}\left(100 \mathrm{MHz}, \mathrm{CDCl}_{3}\right): \delta 161.5(\mathrm{~d}, J=$ $33.5 \mathrm{~Hz}, 1 \mathrm{C}), 146.6$ (d, $J=262.8 \mathrm{~Hz}, 1 \mathrm{C}), 139.1$ (d, $J=4.7 \mathrm{~Hz}, 1 \mathrm{C}), 136.1$ (d, $J=1.9 \mathrm{~Hz}, 1 \mathrm{C}), 129.1$, 128.8, 127.3, $119.2(\mathrm{~d}, J=8.7 \mathrm{~Hz}, 1 \mathrm{C}), 118.8(\mathrm{~d}, J=2.1 \mathrm{~Hz}, 1 \mathrm{C}), 52.4 ;{ }^{19} \mathrm{~F} \mathrm{NMR}\left(376 \mathrm{MHz}, \mathrm{CDCl}_{3}\right)$ : $\delta-128.80$. The spectroscopic data correspond to those previously reported in the literature. ${ }^{[5]}$

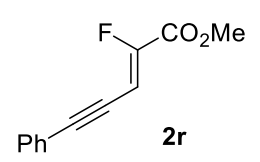

${ }^{19} \mathrm{~F}$ NMR analysis revealed that the $Z / E$ ratio was $12: 1,(Z)-2 \mathbf{r}$ was isolated in $23.6 \mathrm{mg}$ (58\% yield) as yellow solid by column chromatography using PE:EtOAc (20:1, v/v) as the eluent; $\mathrm{Mp} 63-65{ }^{\circ} \mathrm{C} ;{ }^{1} \mathrm{H}$ NMR $\left(400 \mathrm{MHz}, \mathrm{CDCl}_{3}\right): \delta 7.52-7.50(\mathrm{~m}, 2 \mathrm{H}), 7.39-7.33$ $(\mathrm{m}, 3 \mathrm{H}), 6.40(\mathrm{~d}, J=28.4 \mathrm{~Hz}, 1 \mathrm{H}), 3.88(\mathrm{~s}, 3 \mathrm{H}) ;{ }^{13} \mathrm{C} \mathrm{NMR}\left(100 \mathrm{MHz}, \mathrm{CDCl}_{3}\right): \delta 160.6(\mathrm{~d}, J=33.1 \mathrm{~Hz}$, 1C), $153.8(\mathrm{~d}, J=272.1 \mathrm{~Hz}, 1 \mathrm{C}), 131.9,129.4,128.5,122.1$ (d, $J=1.5 \mathrm{~Hz}, 1 \mathrm{C}), 102.4(\mathrm{~d}, J=6.8 \mathrm{~Hz}$, 1C), $100.6(\mathrm{~d}, J=11.1 \mathrm{~Hz}, 1 \mathrm{C}), 80.2,52.8 ;{ }^{19} \mathrm{~F}$ NMR (376 MHz, $\mathrm{CDCl}_{3}$ ): $\delta$-114.51; IR (ATR) v 1732.1, 1504.5, 1300.0, 1240.2, 1118.7, 968.3, 920.1, 860.3, 756.1, $688.6 \mathrm{~cm}^{-1} ; \mathrm{HRMS}(\mathrm{ESI}) \mathrm{m} / \mathrm{z}:[\mathrm{M}+\mathrm{Na}]^{+}$ Calcd for $\mathrm{C}_{12} \mathrm{H}_{9} \mathrm{FNaO}_{2} 227.0479$; Found 227.0481.

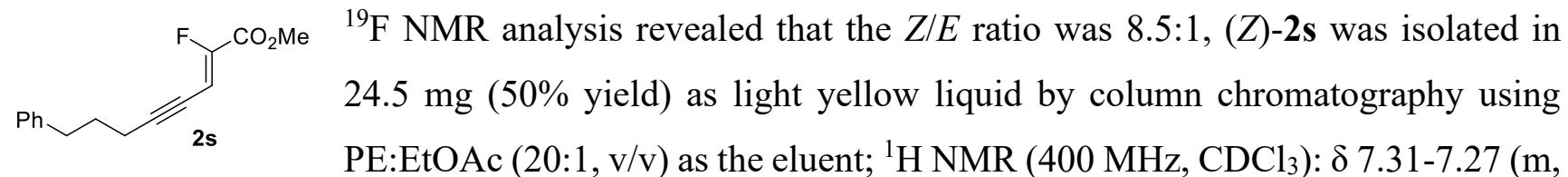
2H), 7.21-7.18 (m, 3H), $6.18(\mathrm{dt}, J=28.8 \mathrm{~Hz}, 2.4 \mathrm{~Hz}, 1 \mathrm{H}), 3.85$ (s, 3H), 2.74 (t, $J=7.6 \mathrm{~Hz}, 2 \mathrm{H}), 2.44-$ 2.40 (m, 2H), 1.94-1.86 (m, 2H); $\left.{ }^{13} \mathrm{C} \mathrm{NMR} \mathrm{(100} \mathrm{MHz,} \mathrm{CDCl}_{3}\right): \delta 160.8$ (d, $\left.J=33.2 \mathrm{~Hz}, 1 \mathrm{C}\right), 154.0$ (d, $J=269.2 \mathrm{~Hz}, 1 \mathrm{C}), 141.2,128.5,128.4,126.0,104.5$ (d, $J=6.7 \mathrm{~Hz}, 1 \mathrm{C}), 101.2$ (d, $J=10.9 \mathrm{~Hz}, 1 \mathrm{C})$, 
72.3, 52.7, 34.7, 29.8, 19.3; $\left.{ }^{19} \mathrm{~F} \mathrm{NMR} \mathrm{(376} \mathrm{MHz,} \mathrm{CDCl}_{3}\right): \delta$-116.97; IR (ATR) v 2974.2, 1762.9, 1703.1, 1319.3, 1265.3, 1242.2, 1203.6, 1047.4, 879.5, $740.7 \mathrm{~cm}^{-1}$; HRMS (ESI) m/z: $[\mathrm{M}+\mathrm{Na}]^{+}$Calcd for $\mathrm{C}_{15} \mathrm{H}_{15} \mathrm{FNaO}_{2} 269.0948$; Found 269.0936.

$\mathrm{F}_{-} \mathrm{CO}_{2} \mathrm{Me}$ Product $2 \mathrm{t}$ was obtained in $28.1 \mathrm{mg}$ ( $55 \%$ yield) as light yellow liquid by column ${ }_{\mathrm{Ph}}{ }_{\mathrm{Ph}}$ chromatography using PE:EtOAc (20:1, v/v) as the eluent; ${ }^{1} \mathrm{H}$ NMR $\left(400 \mathrm{MHz}, \mathrm{CDCl}_{3}\right): \delta$ 2t $\quad 7.38-7.36(\mathrm{~m}, 3 \mathrm{H}), 7.35-7.30(\mathrm{~m}, 5 \mathrm{H}), 7.21-7.18(\mathrm{~m}, 2 \mathrm{H}), 3.66(\mathrm{~s}, 3 \mathrm{H}) ;{ }^{13} \mathrm{C} \mathrm{NMR}(100 \mathrm{MHz}$, $\left.\mathrm{CDCl}_{3}\right): \delta 161.8(\mathrm{~d}, J=35.1 \mathrm{~Hz}, 1 \mathrm{C}), 143.9$ (d, $\left.J=261.6 \mathrm{~Hz}, 1 \mathrm{C}\right), 137.0(\mathrm{~d}, J=4.6 \mathrm{~Hz}, 1 \mathrm{C}), 136.2$ (d, $J=1.7 \mathrm{~Hz}, 1 \mathrm{C}), 133.7$ (d, $J=11.9 \mathrm{~Hz}, 1 \mathrm{C}), 130.0$ (d, $J=5.4 \mathrm{~Hz}, 1 \mathrm{C}), 129.5$ (d, $J=3.2 \mathrm{~Hz}, 1 \mathrm{C}), 129.0$, 128.3, 128.2, 128.2, 52.2; ${ }^{19} \mathrm{~F}$ NMR (376 MHz, $\mathrm{CDCl}_{3}$ ): $\delta$-122.81; IR (ATR) v 2358.9, 1728.2, 1325.1, 1269.2, 1197.8, 1132.2, 1051.2, 881.5, 742.6, $698.2 \mathrm{~cm}^{-1}$; HRMS (ESI) m/z: $[\mathrm{M}+\mathrm{Na}]^{+}$Calcd for $\mathrm{C}_{16} \mathrm{H}_{13} \mathrm{FNaO}_{2}$ 279.0792; Found 279.0790.

\section{Product elaboration}

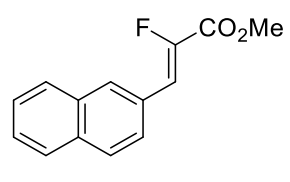

$2 \mathbf{a}$

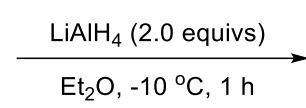

$\mathrm{Et}_{2} \mathrm{O},-10^{\circ} \mathrm{C}, 1 \mathrm{~h}$

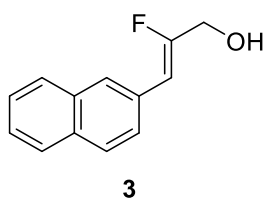

In a flame-dried Schlenk tube $(10 \mathrm{~mL})$ containing a stirring bar was charged with ester $2 \mathbf{a}(23.0 \mathrm{mg}$, $0.1 \mathrm{mmol}, 1.0$ equiv) and $\mathrm{LiAlH}_{4}(7.6 \mathrm{mg}, 0.2 \mathrm{mmol}, 2.0$ equivs $)$. After which, the tube was purged and back-filled with nitrogen (this operation was repeated three times). To this mixture was added anhydrous $\mathrm{Et}_{2} \mathrm{O}(2 \mathrm{~mL})$ via syringe at $-10{ }^{\circ} \mathrm{C}$ and left stirred for $1 \mathrm{~h}$. And monitored by TLC until full conversion to the product was observed. the reaction mixture was quenched with water $(6 \mu \mathrm{L}), 15 \% \mathrm{NaOH}(6 \mu \mathrm{L})$ and water $(17 \mu \mathrm{L})$ sequentially. Then the mixture was filtered and concentrated in vacuo. The residue was purified by chromatography on silica gel (petroleum ether/ethyl acetate $=3 / 1, \mathrm{v} / \mathrm{v}$ ) to give the product 3 (16.7 mg, 83\% yield) as a white solid. ${ }^{1} \mathrm{H}$ NMR (400 MHz, $\left.\mathrm{CDCl}_{3}\right): \delta 7.93(\mathrm{~s}, 1 \mathrm{H}), 7.82-7.79$ (m, 3H), 7.67 (dd, $J=8.8 \mathrm{~Hz}, 1.6 \mathrm{~Hz}, 1 \mathrm{H}), 7.48-7.44$ (m, 2H), 5.94 (d, $J=38.8 \mathrm{~Hz}, 1 \mathrm{H}), 4.34$ (d, $J=$ $14.4 \mathrm{~Hz}, 2 \mathrm{H}), 1.81(\mathrm{br}, 1 \mathrm{H}) ;{ }^{13} \mathrm{C} \mathrm{NMR}\left(100 \mathrm{MHz}, \mathrm{CDCl}_{3}\right): \delta 158.4$ (d, $\left.J=265.5 \mathrm{~Hz}, 1 \mathrm{C}\right), 133.4,132.6$ (d, $J=1.9 \mathrm{~Hz}, 1 \mathrm{C}), 130.2$ (d, $J=2.9 \mathrm{~Hz}, 1 \mathrm{C}), 128.1,128.1,127.9$ (d, $J=7.1 \mathrm{~Hz}, 1 \mathrm{C}), 127.6,126.5$ (d, $J=7.6 \mathrm{~Hz}, 1 \mathrm{C}), 126.2,126.1,107.6(\mathrm{~d}, J=6.5 \mathrm{~Hz}, 1 \mathrm{C}), 62.0(\mathrm{~d}, J=32.5 \mathrm{~Hz}, 1 \mathrm{C}) ;{ }^{19} \mathrm{~F}$ NMR $(376 \mathrm{MHz}$, $\left.\mathrm{CDCl}_{3}\right): \delta-112.96$. The spectroscopic data correspond to those previously reported in the literature. ${ }^{6}$

\footnotetext{
${ }^{6}$ Zhou, J.; Jiang, X.; Jin, C.; Guo, Z.; Su, B.; Su, W. Eur. J. Org. Chem., 2017, 3631.
} 


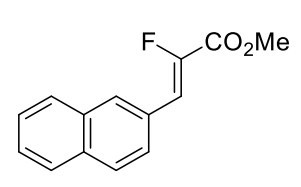

$2 a$

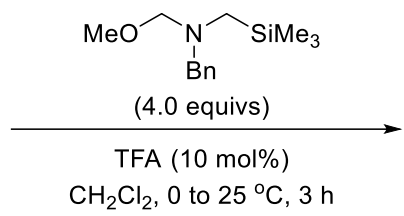

$\mathrm{CH}_{2} \mathrm{Cl}_{2}, 0$ to $25^{\circ} \mathrm{C}, 3 \mathrm{~h}$

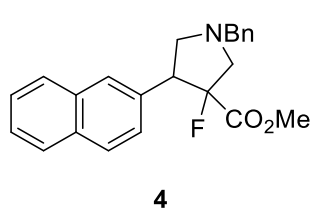

In a flame-dried Schlenk tube $(10 \mathrm{~mL})$ containing a stirring bar, a solution of $\mathbf{2 a}(23.0 \mathrm{mg}, 0.2 \mathrm{mmol}$, 1.0 equiv), $N$-benzyl-1-methoxy- $N$-((trimethylsilyl)methyl)methanamine $(95.0 \mathrm{mg}, 0.4 \mathrm{mmol}, 4.0$ equivs) in $\mathrm{CH}_{2} \mathrm{Cl}_{2}(1.0 \mathrm{~mL})$ and cooled to $0{ }^{\circ} \mathrm{C}$, followed by the addition of TFA (1.2 $\mathrm{mg}, 0.01 \mathrm{mmol}$, $10 \mathrm{~mol} \%$ ). Then the mixture was stirred at $25^{\circ} \mathrm{C}$ for a further $3 \mathrm{~h}$. After the completion of the reaction was observed by TLC. The mixture was concentrated in vacuo and purified by a silica gel flash column chromatography (petroleum ether/EtOAc $=7 / 1, \mathrm{v} / \mathrm{v})$ to give the pure desired product $4(29.6 \mathrm{mg}, 82 \%$ yield) as light yellow liquid. ${ }^{1} \mathrm{H}$ NMR $\left(400 \mathrm{MHz}, \mathrm{CDCl}_{3}\right): \delta$ 7.81-7.76 (m, 3H), 7.71 (s, $\left.1 \mathrm{H}\right), 7.46-7.39$ (m, 5H), 7.36-7.33 (m, 2H), 7.29-7.27 (m, 1H), 4.10-4.00 (m, 1H), 3.91-3.80 (m, 2H), 3.77 (s, 3H), 3.61-3.51 (m, 1H), 3.34-3.30 (m, 1H), 3.26-3.21 (m, 1H), 3.16-3.06 (m, 1H); ${ }^{13} \mathrm{C}$ NMR $(100 \mathrm{MHz}$, $\left.\mathrm{CDCl}_{3}\right): \delta 170.7(\mathrm{~d}, J=27.7 \mathrm{~Hz}, 1 \mathrm{C}), 138.4,133.2,132.8,132.2(\mathrm{~d}, J=3.5 \mathrm{~Hz}, 1 \mathrm{C}), 128.7,128.4,128.1$, 127.9, 127.8, 127.6, 127.3, 127.1 (d, $J=1.9 \mathrm{~Hz}, 1 \mathrm{C}), 126.0,125.9,100.2$ (d, $J=199.0 \mathrm{~Hz}, 1 \mathrm{C}), 64.0$ $(\mathrm{d}, J=24.4 \mathrm{~Hz}, 1 \mathrm{C}), 60.2,57.9,52.8,52.7(\mathrm{~d}, J=19.9 \mathrm{~Hz}, 1 \mathrm{C}) ;{ }^{19} \mathrm{~F} \mathrm{NMR}\left(376 \mathrm{MHz}, \mathrm{CDCl}_{3}\right): \delta-160.75$; IR (ATR) v 1759.1, 1475.5, 1394.5, 1319.3, 1267.2, 1124.5, 1066.6, 1051.2, 742.6, $706.0 \mathrm{~cm}^{-1}$; HRMS (ESI) $\mathrm{m} / \mathrm{z}:[\mathrm{M}+\mathrm{H}]^{+}$Calcd for $\mathrm{C}_{23} \mathrm{H}_{23} \mathrm{O}_{2} \mathrm{NF} 364.1707$; Found: 364.1698 . 


\section{Cyclic voltammetry (CV) analysis}

Cyclic voltammograms were recorded with a Bio-logic VSP Potentiostat/Galvanostat equipped with electrochemical analysis software at room temperature in DMF. $n$-Bu4NI $(0.07 \mathrm{M})$ was used as the supporting electrolyte, and a Pt plate electrode was used as the working electrode. The auxiliary electrode was a Pt pillar. All potentials are referenced against the $\mathrm{Ag} / \mathrm{AgNO}_{3}\left(0.1 \mathrm{M}\right.$ in $\left.\mathrm{CH}_{3} \mathrm{CN}\right)$ redox couple. The scan rate is $100 \mathrm{mV} \mathrm{s}^{-1}$.

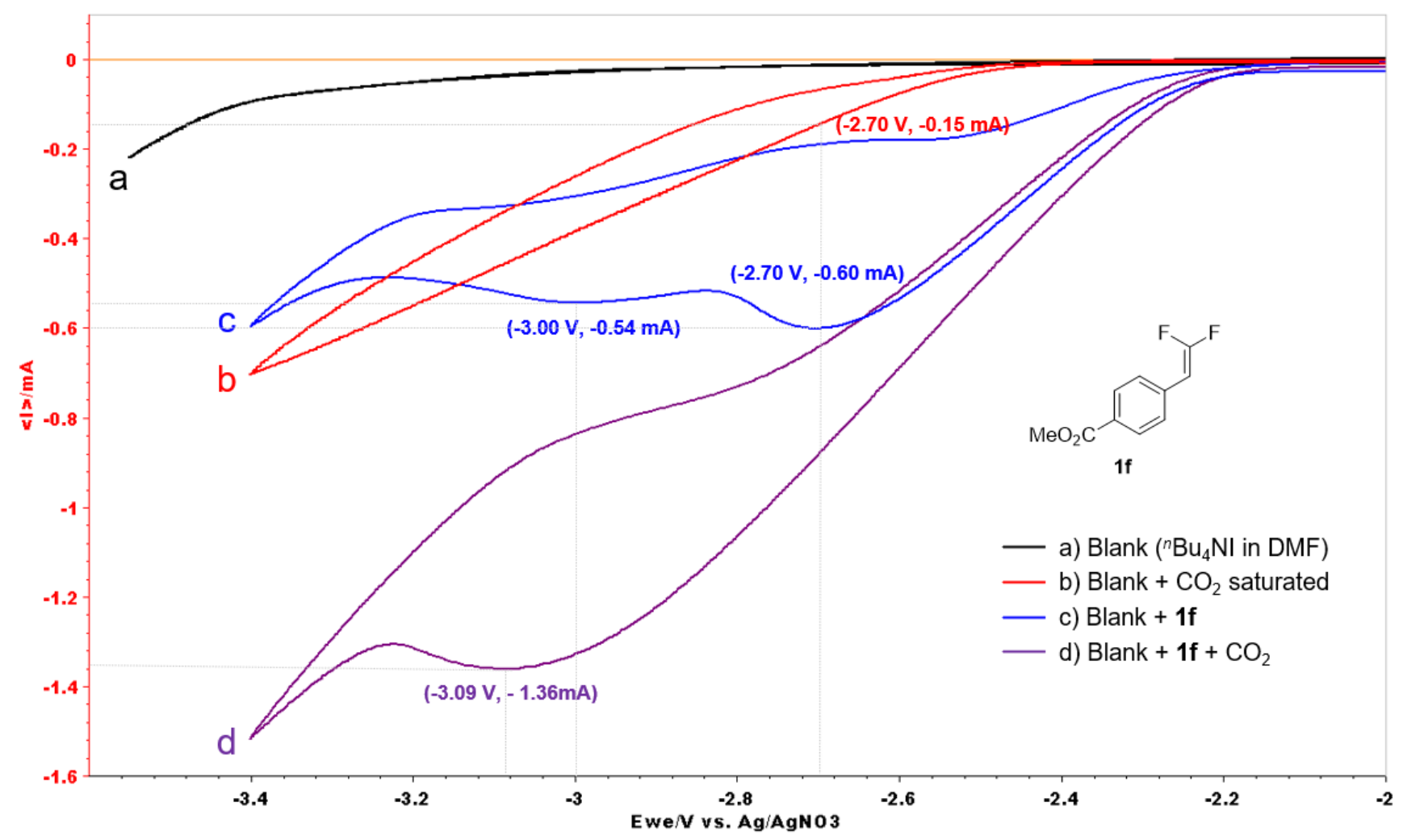

Figure S1. Cyclic voltammograms recorded on a Pt plate electrode. The scan rate is $100 \mathrm{mVs}^{-1}$ : (a) DMF containing 0.07 M n-Bu $\mathrm{NI}$; (b) DMF containing 0.07 $\mathrm{M} n$-Bu $\mathrm{Bu}_{4} \mathrm{NI}$, after $\mathrm{CO}_{2}$ saturated; (c) DMF containing $0.07 \mathrm{M} n$-Bu $\mathrm{Bu}_{4} \mathrm{NI}$ after addition of $0.02 \mathrm{M}$ 1f; (d) DMF containing $0.07 \mathrm{M} n$-Bu $\mathrm{Bu}_{4} \mathrm{NI}$ after addition of $0.02 \mathrm{M} \mathbf{1 f}$ and $\mathrm{CO}_{2}$ saturated.

For the CV of methyl 4-(2,2-difluorovinyl)benzoate 1 ff (Figure S1), a one-electron reduction peak in the potential at $-2.70 \mathrm{~V}$ and the second one at $-3.00 \mathrm{~V}$ was observed (blue line). While at the potential of $-2.70 \mathrm{~V}$, the reduction current of $\mathrm{CO}_{2}$ is $0.15 \mathrm{~mA}$ (red line), indicating that $\mathbf{1 f}$ should be easier to be reduced than $\mathrm{CO}_{2}$. After the solution of $\mathbf{1} \mathbf{f}$ was saturated with $\mathrm{CO}_{2}$ (purple line), only one reduction peak was observed at $-3.09 \mathrm{~V}$ with the peak current increased from 0.60 to $1.36 \mathrm{~mA}$ (about 2.3 times), indicating a fast interaction of the one-electron reduction species with $\mathrm{CO}_{2}$. 


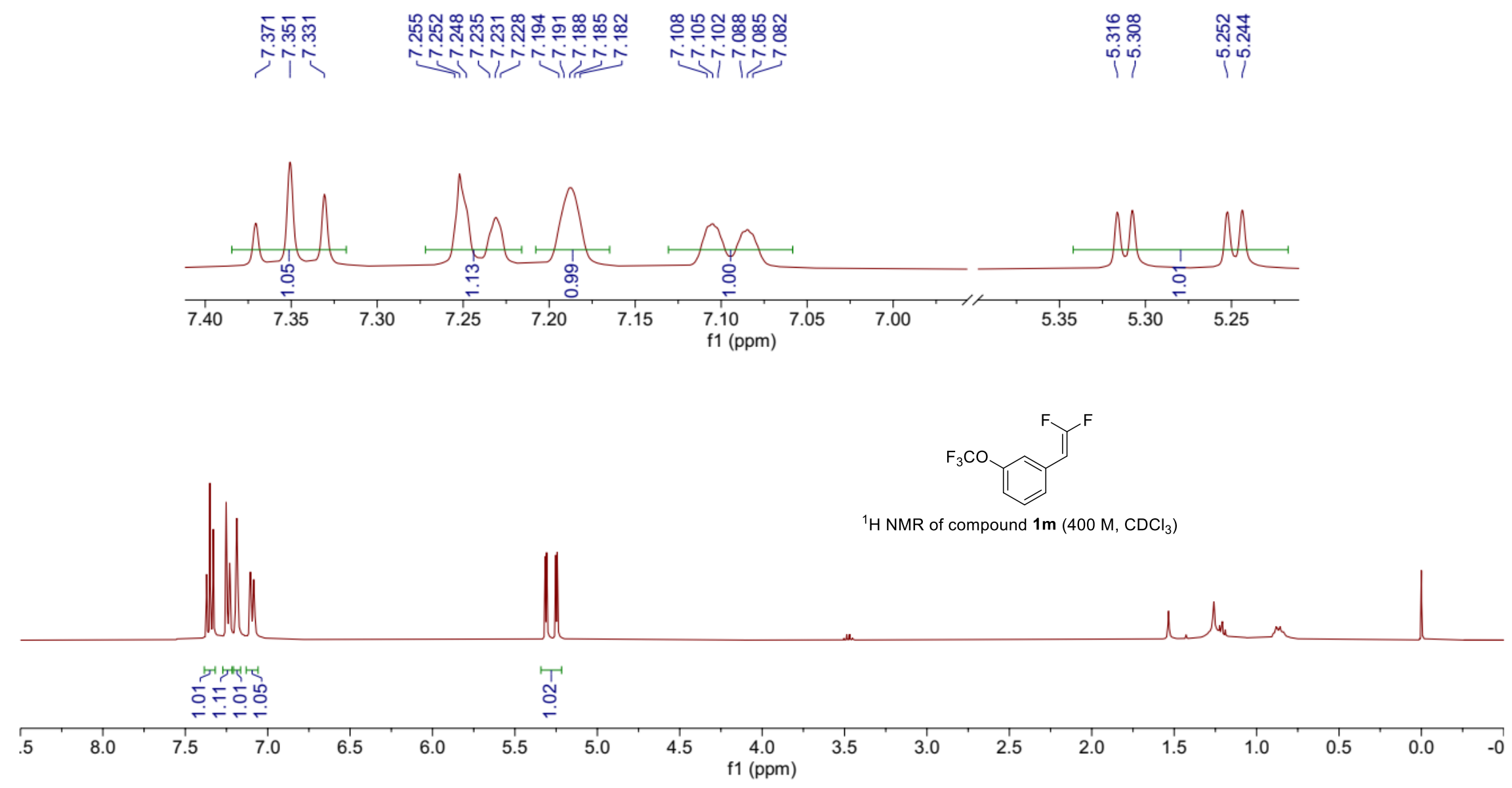


XSL-XJ-32-400M-4. 11. fid

XSL-XJ-32-400M-4 F

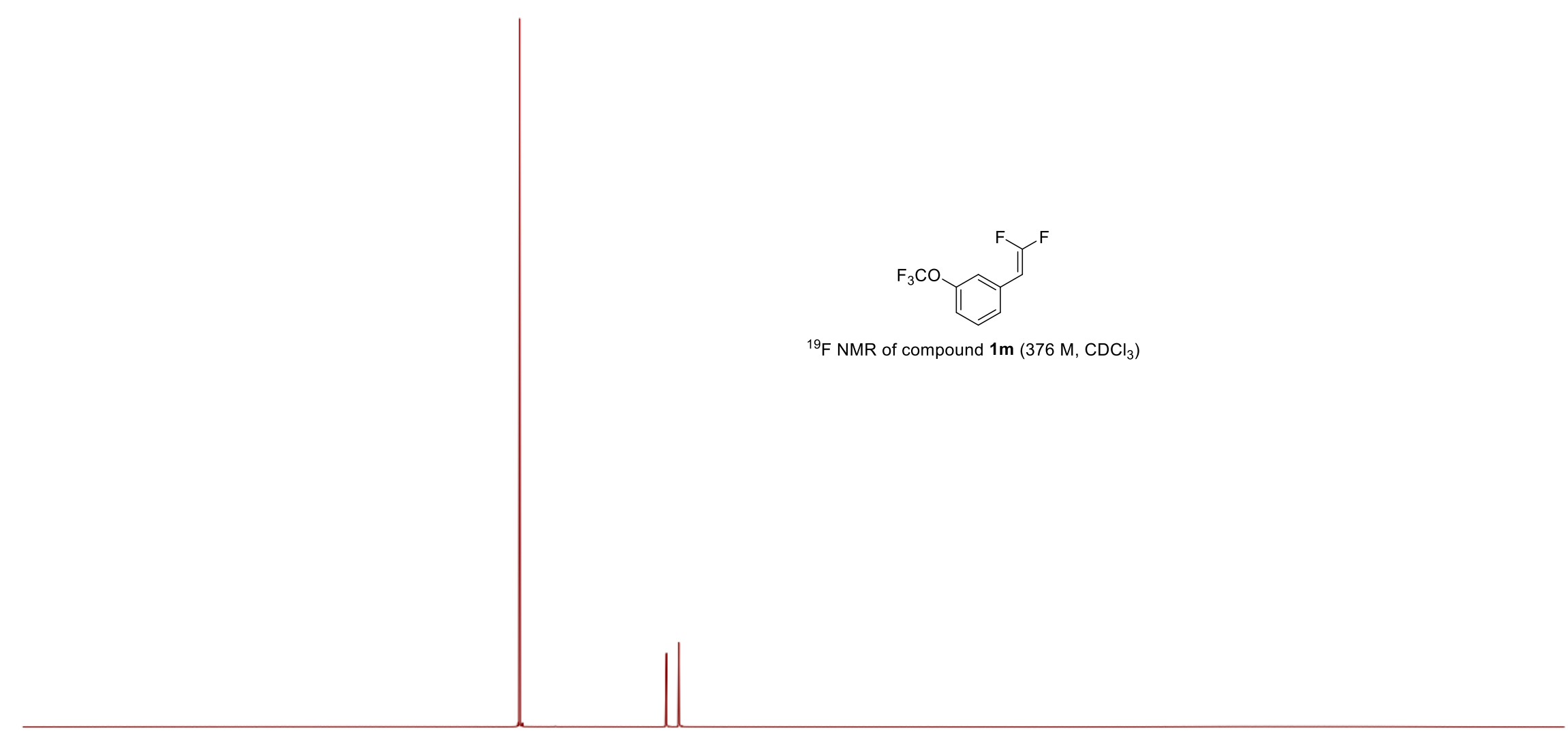

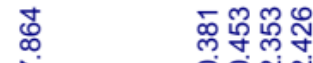

ए क्षळ

${ }^{19} \mathrm{~F} \mathrm{NMR}$ of compound $1 \mathrm{~m}\left(376 \mathrm{M}, \mathrm{CDCl}_{3}\right)$

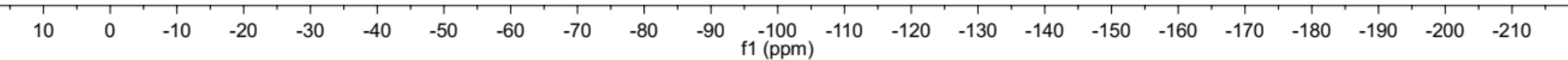




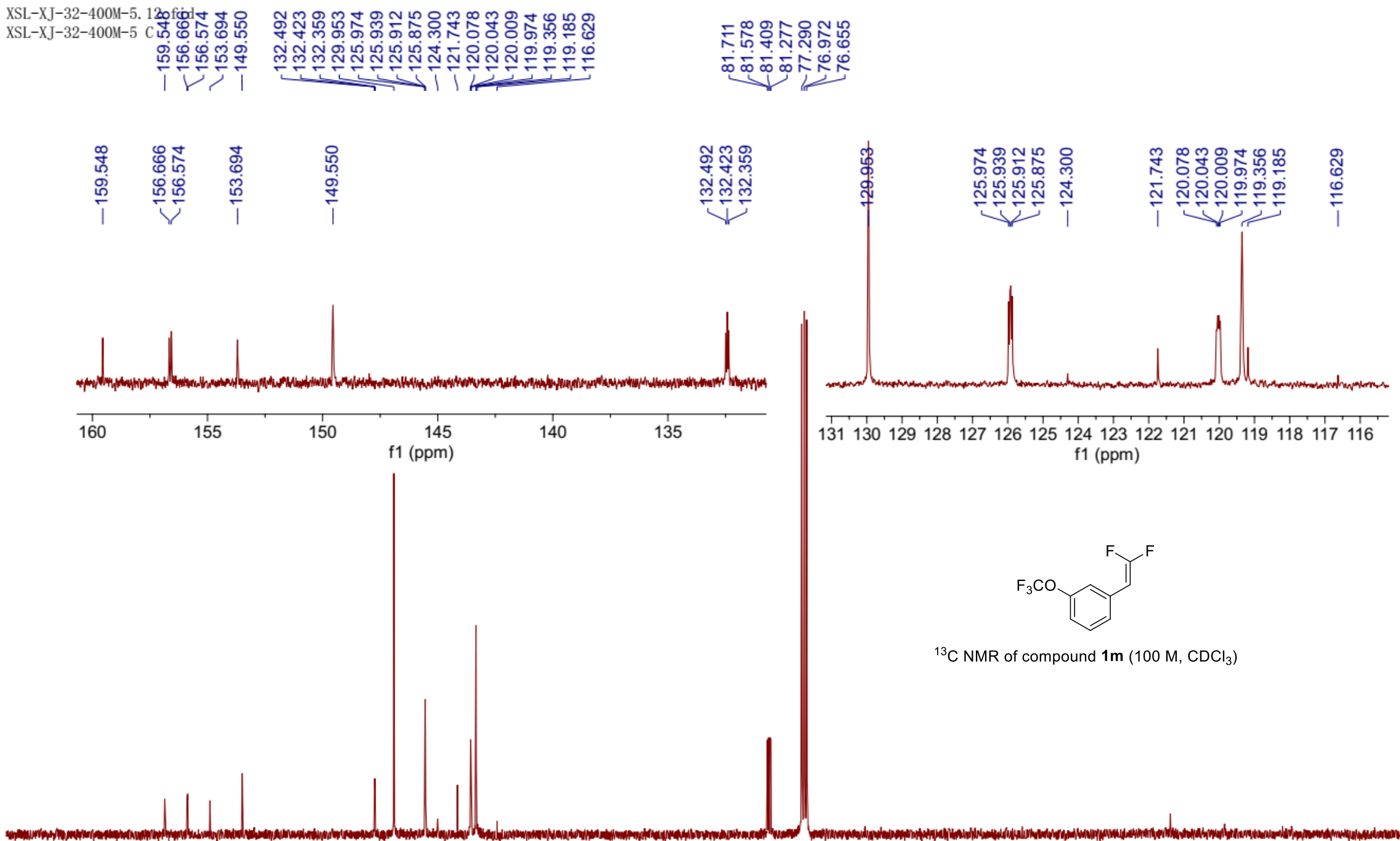

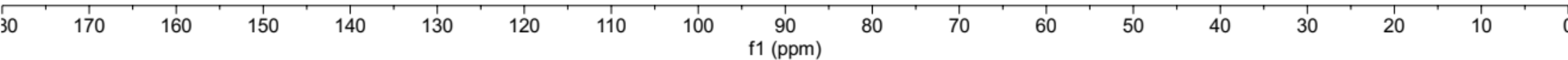




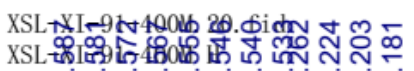

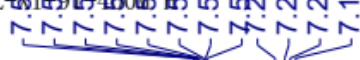

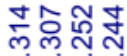

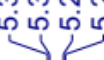

†
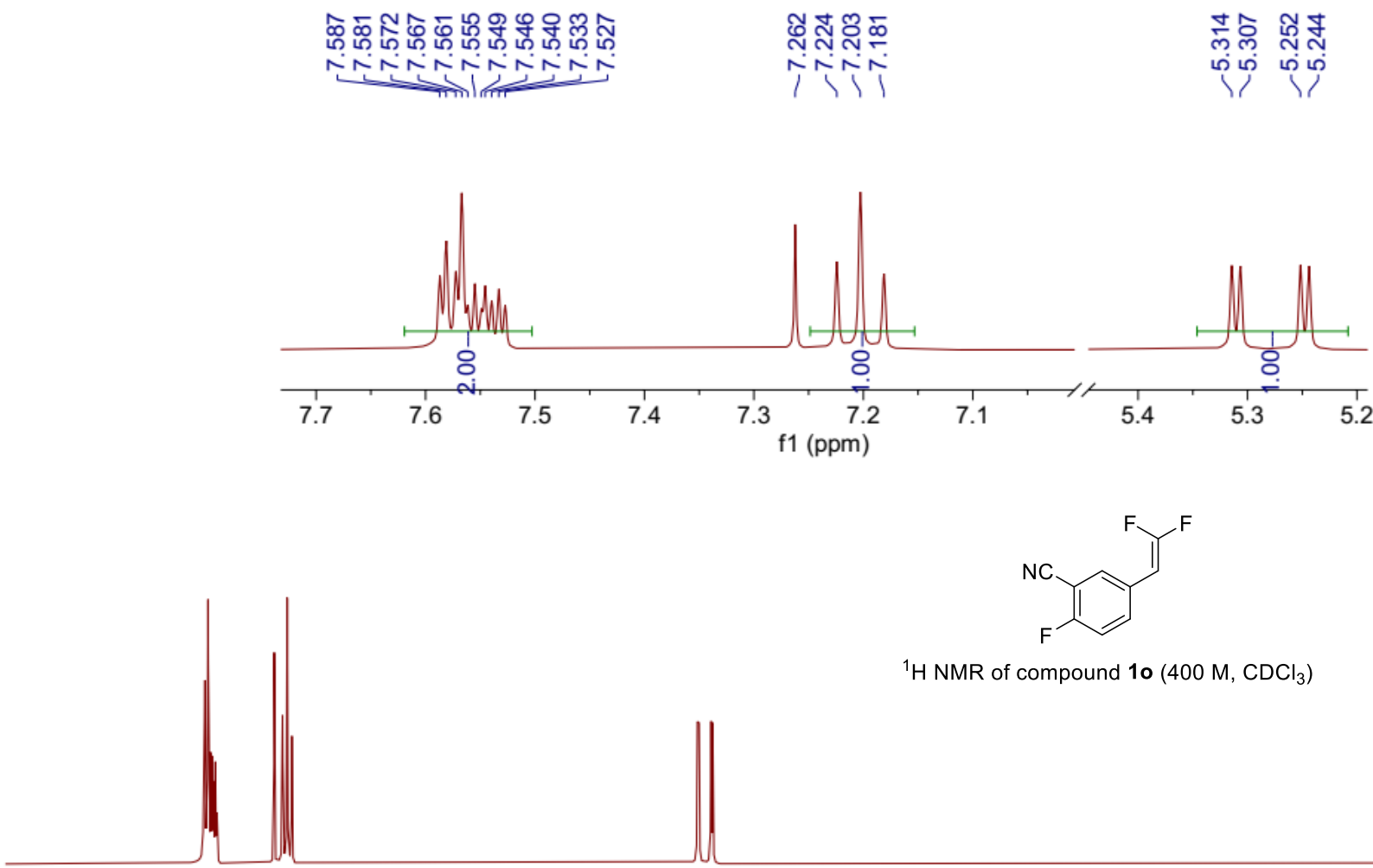

H NMR of compound $10\left(400 \mathrm{M}, \mathrm{CDCl}_{3}\right)$

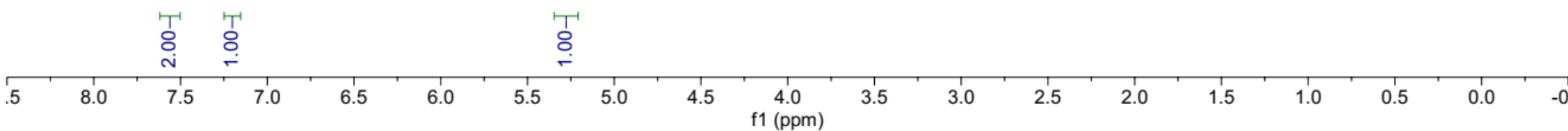


XSL-XI-91-400M. 21. fid

XSL-XI-91-400M

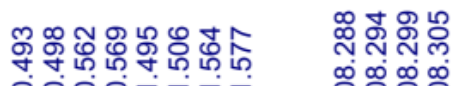

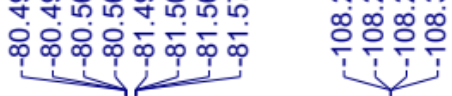
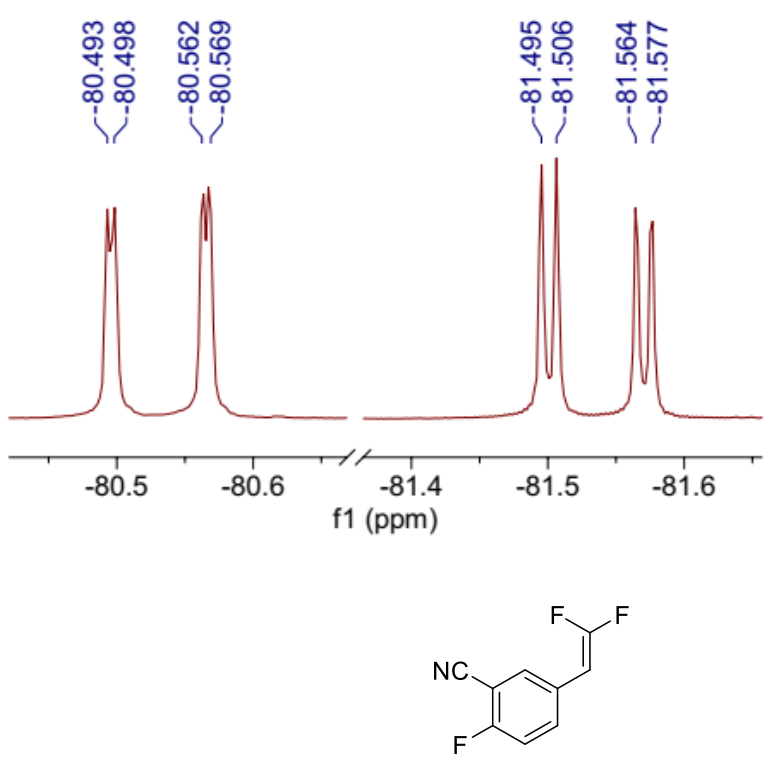

${ }^{19} \mathrm{~F}$ NMR of compound $10\left(376 \mathrm{M}, \mathrm{CDCl}_{3}\right)$

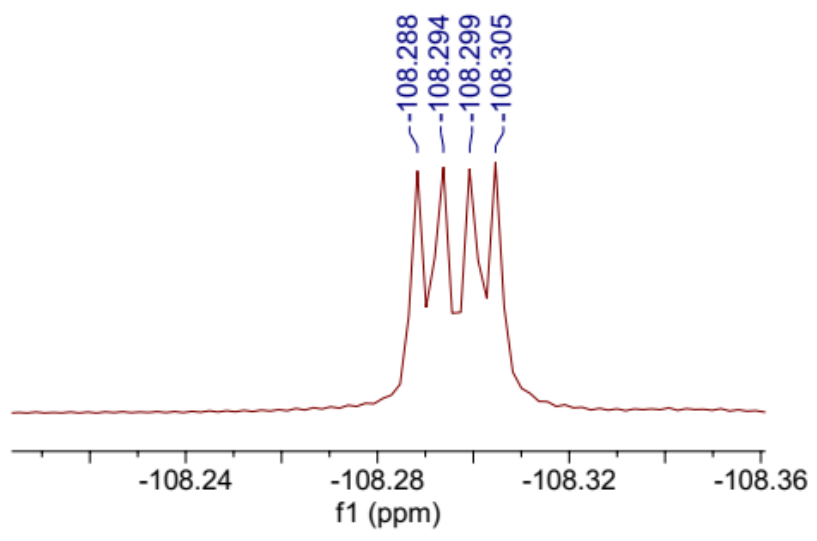


Xsb-

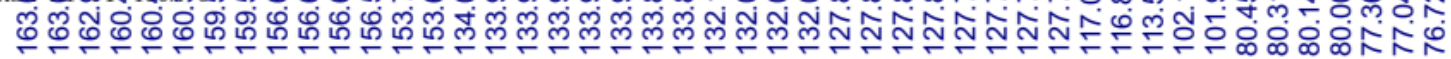

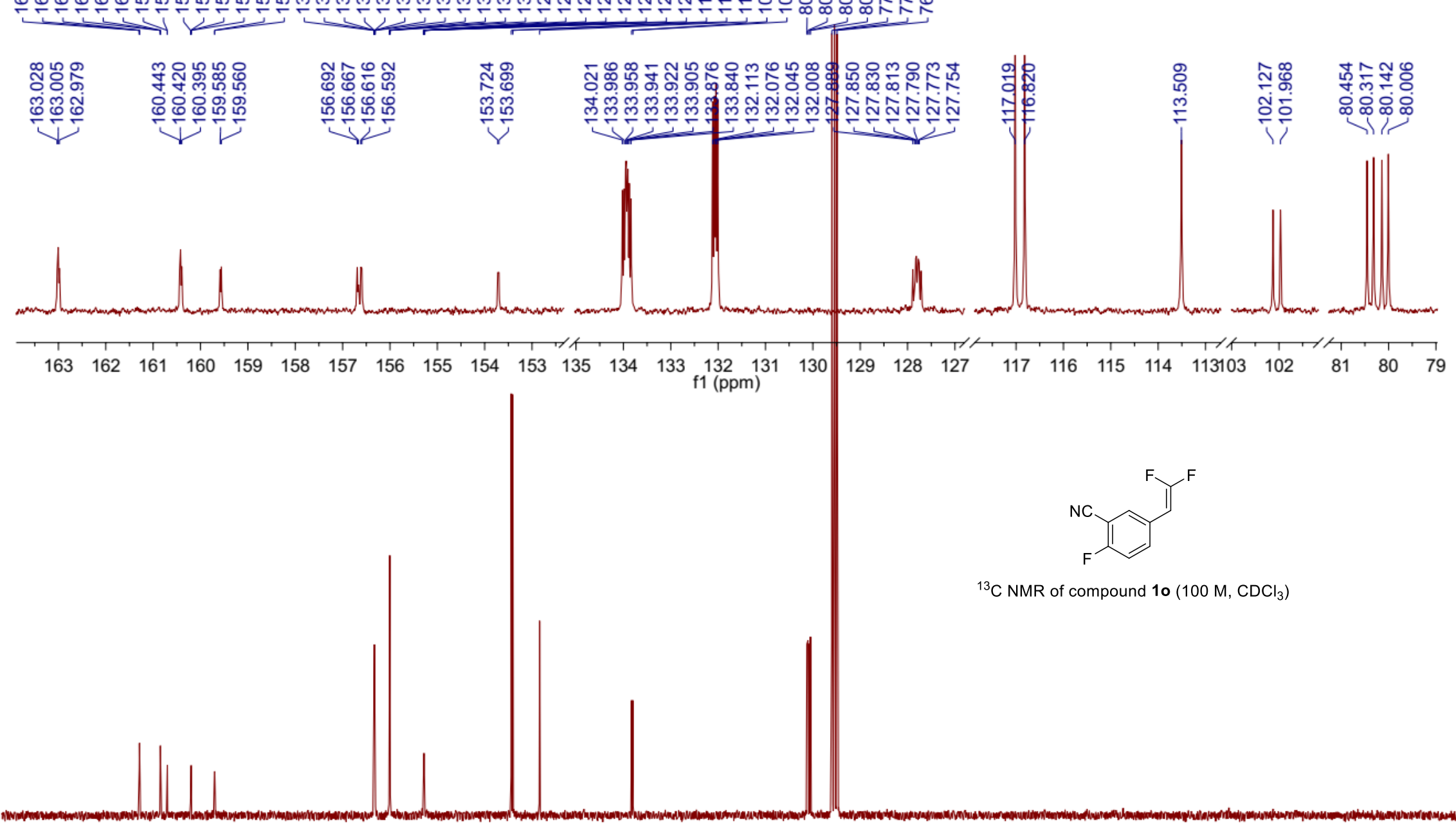

160

150

140

130

120

110

100

90
$\mathrm{f} 1(\mathrm{ppm})$

80

70

60

50

40

30

20

10 


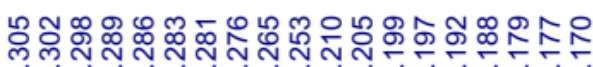

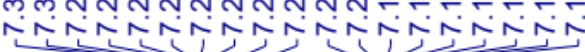

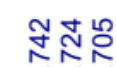
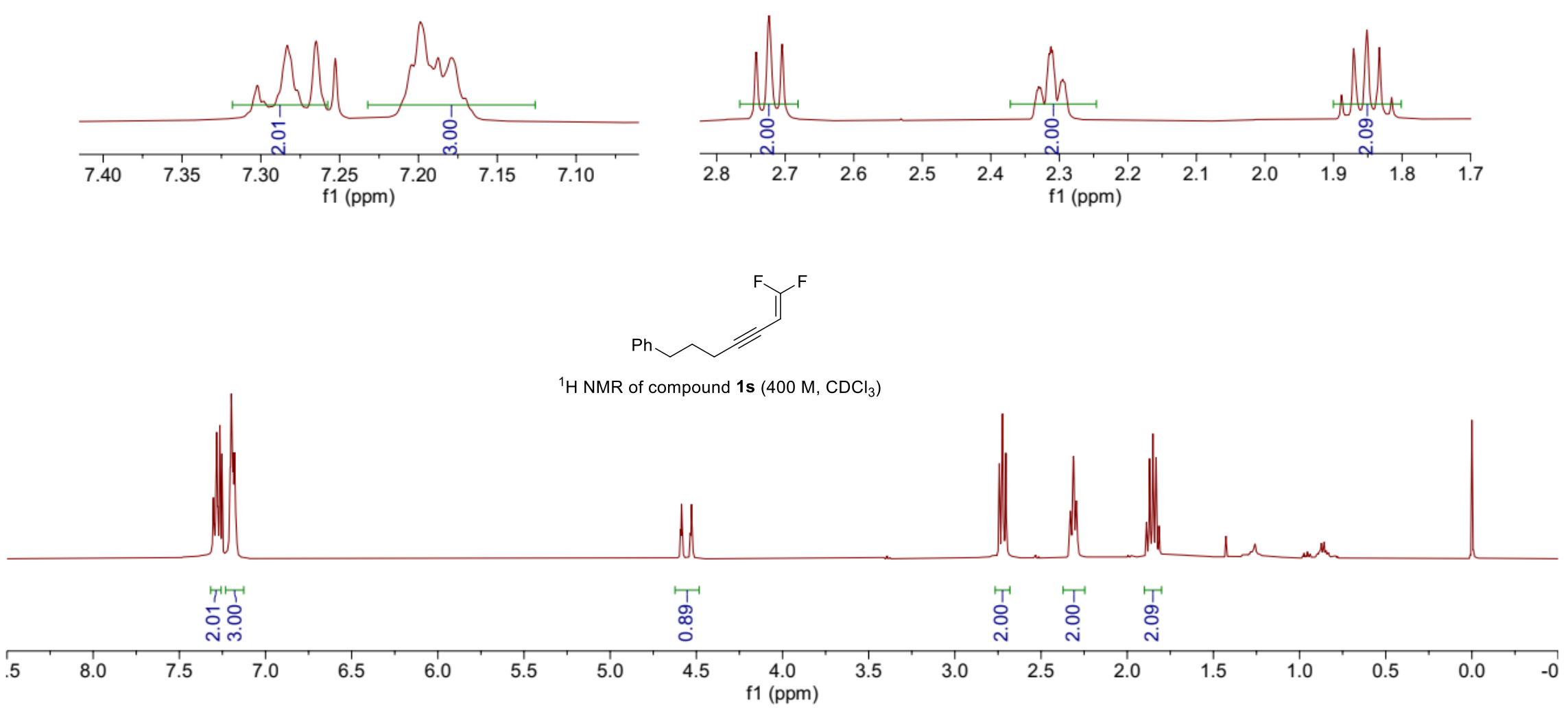
XSL-XJ-38-400M. 11. fid

XSL-XJ-38-400M

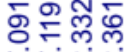

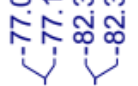

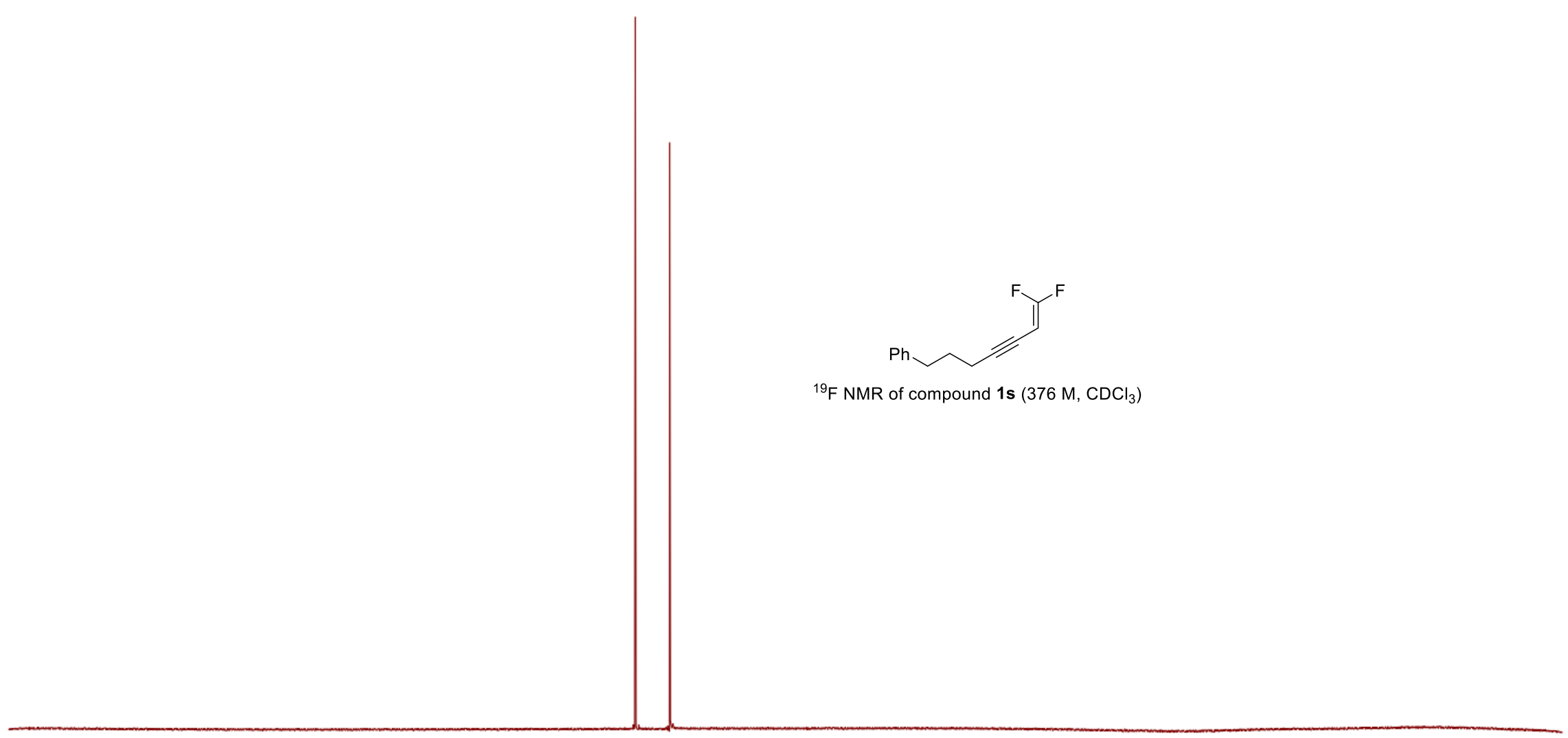

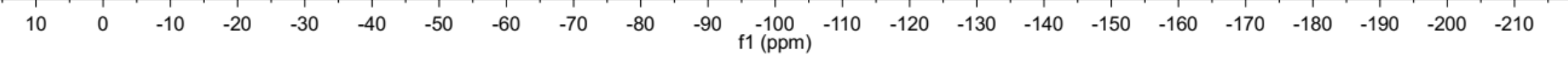



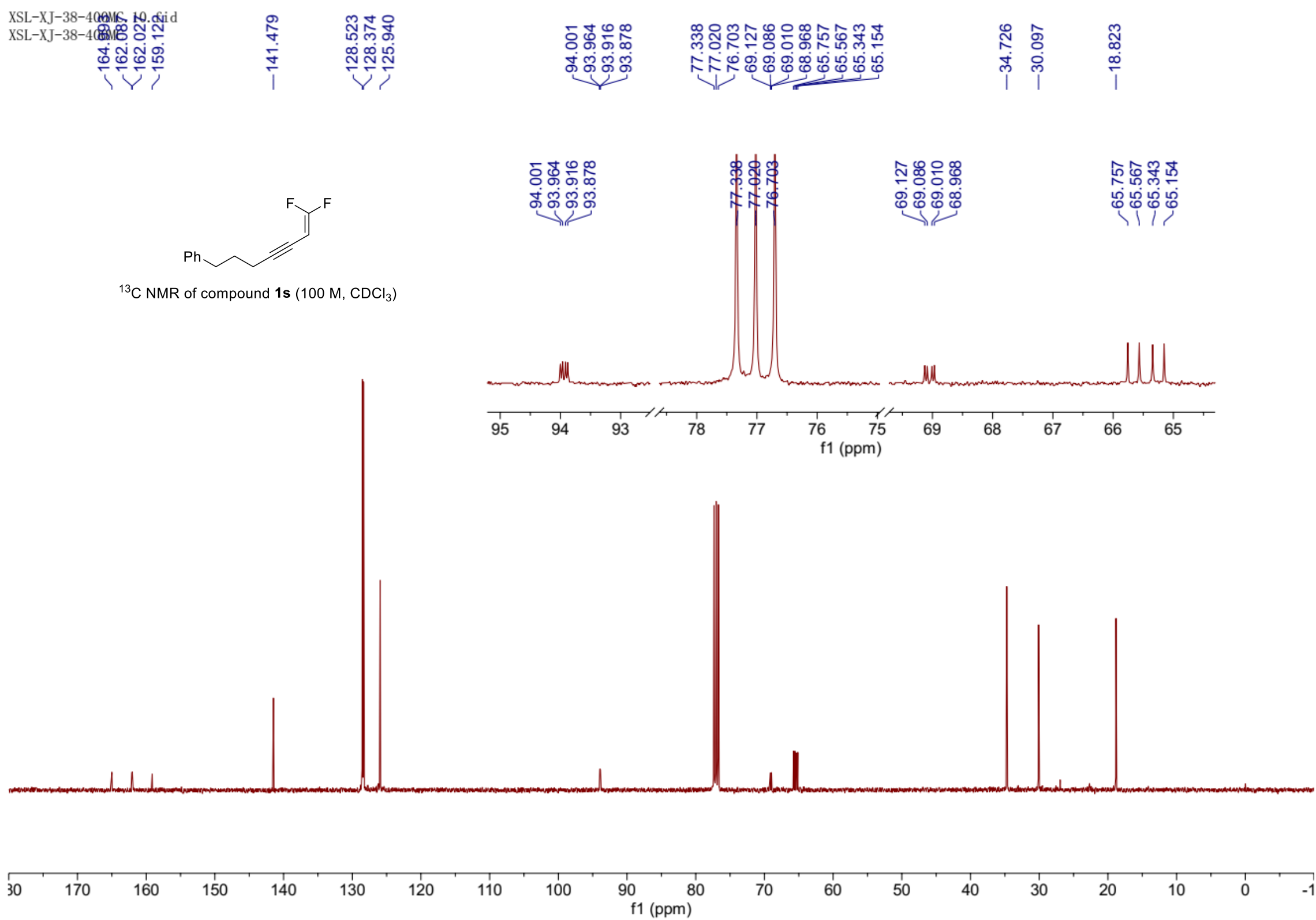


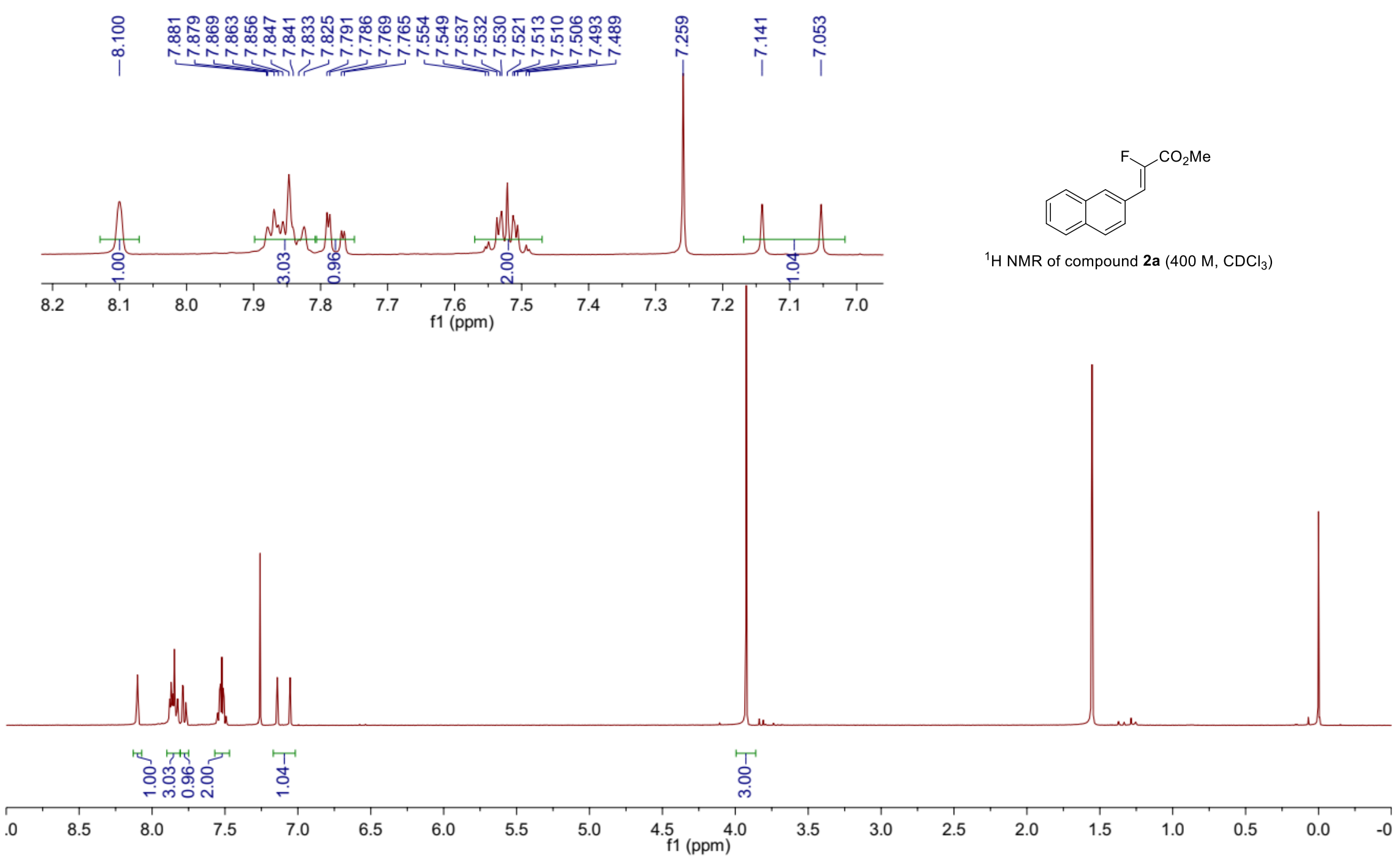


XSL-XI-55-2-400M. 11. fid

XSL-XI-55-2-400M F

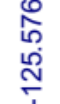

${ }^{19} \mathrm{~F} \mathrm{NMR}$ of compound $2 \mathrm{a}\left(376 \mathrm{M}, \mathrm{CDCl}_{3}\right)$

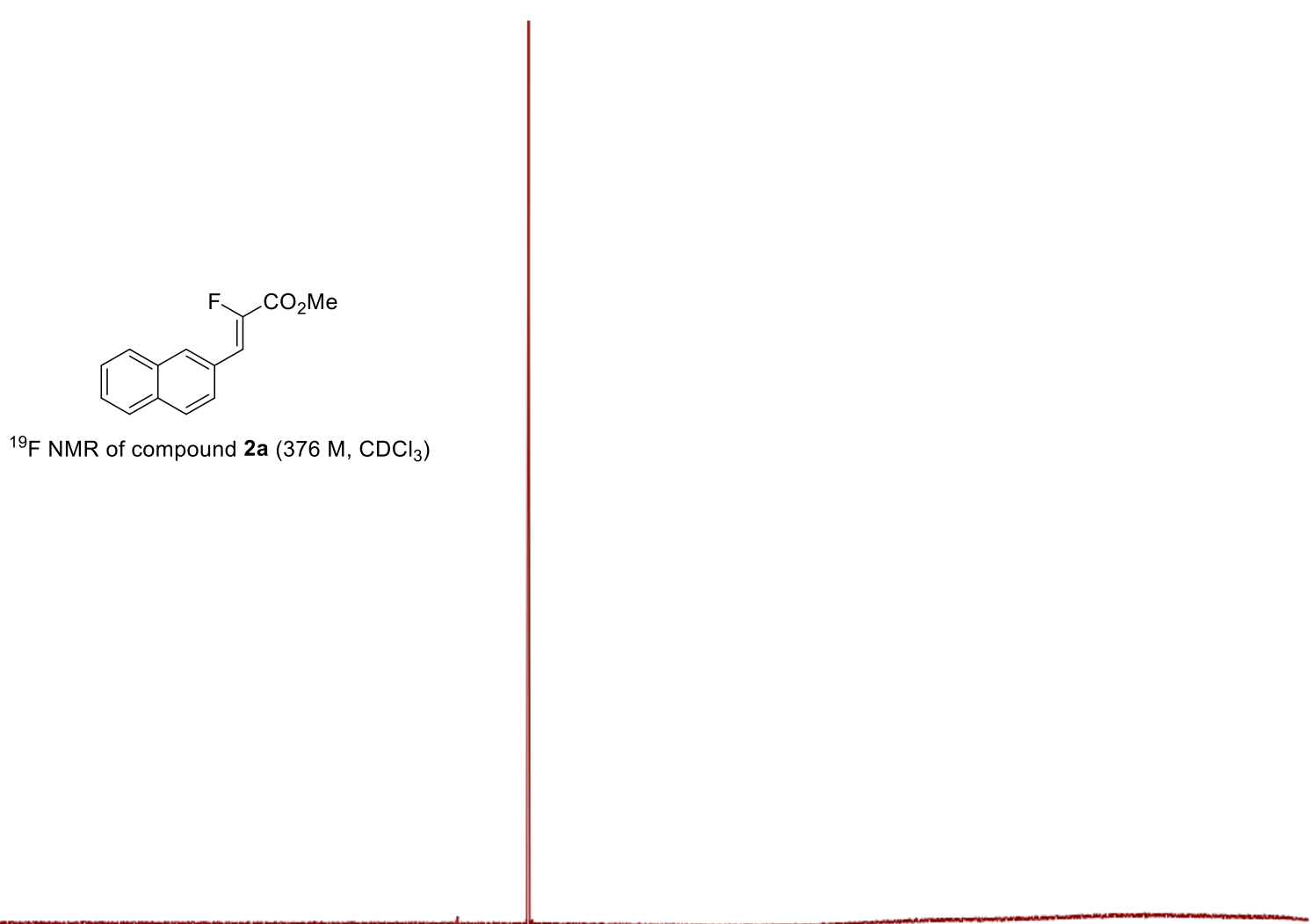

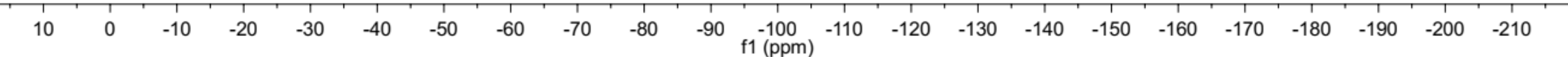




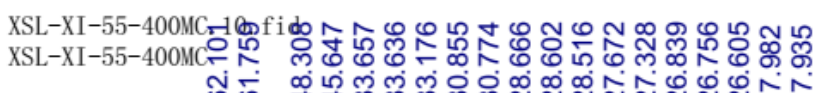

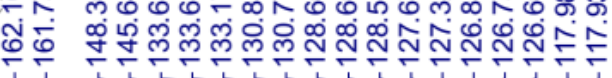

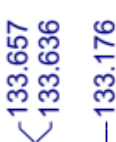

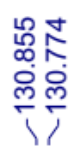

छํํำ

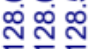

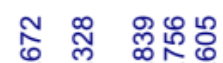

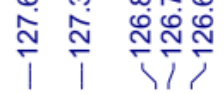

广

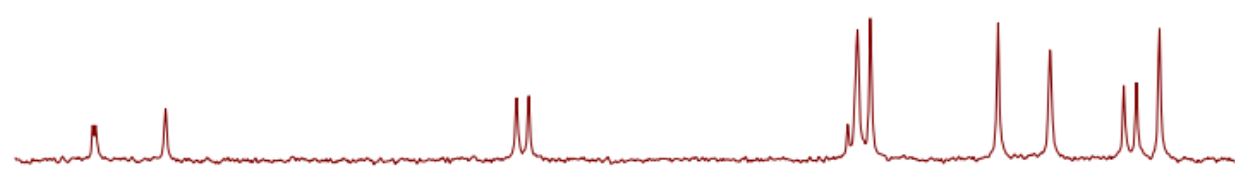

134.0133 .5133 .0132 .5132 .0131 .5131 .0130 .5130 .0129 .5129 .0128 .5128 .0127 .5127 .0126 .5 f1 (ppm)

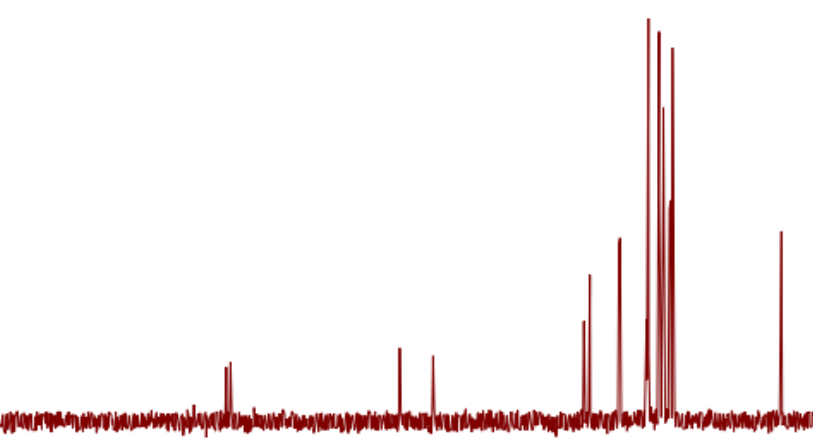

$30 \quad 170$

160

150

140

130

120

110

100

90
$\mathrm{f} 1(\mathrm{ppm})$

80

70

60

50

40

30 


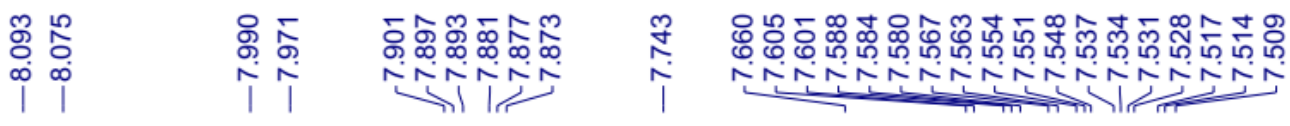
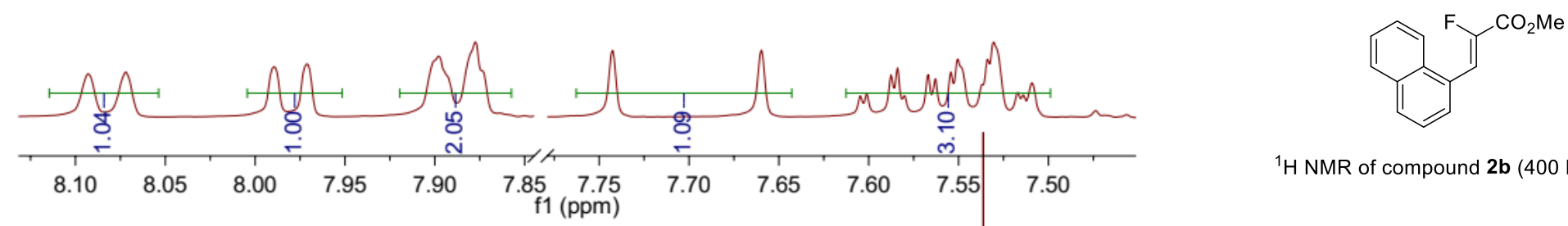

${ }^{1} \mathrm{H}$ NMR of compound $\mathbf{2 b}\left(400 \mathrm{M}, \mathrm{CDCl}_{3}\right)$

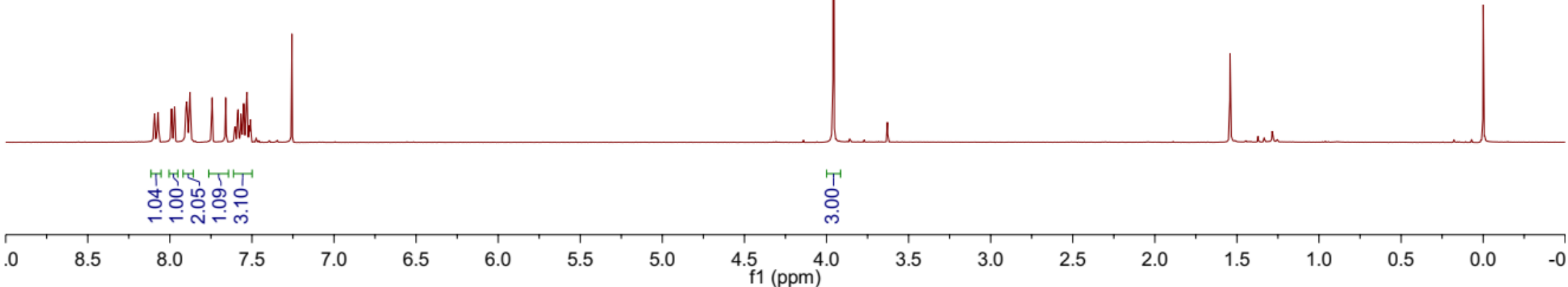


XSL-XI-83-P. 11. fid

XSL-XI-83-P F

${ }^{19} \mathrm{~F}$ NMR of compound $\mathbf{2 b}\left(376 \mathrm{M}, \mathrm{CDCl}_{3}\right)$ $\frac{9}{4}$

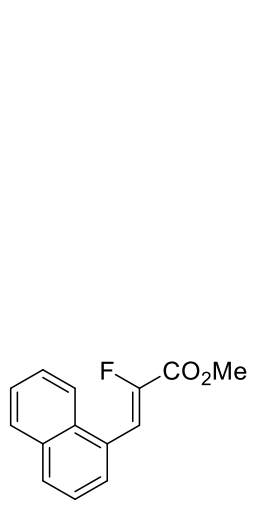

$\frac{i}{i}$

2b (376 M, $\left.\mathrm{CDCl}_{3}\right)$

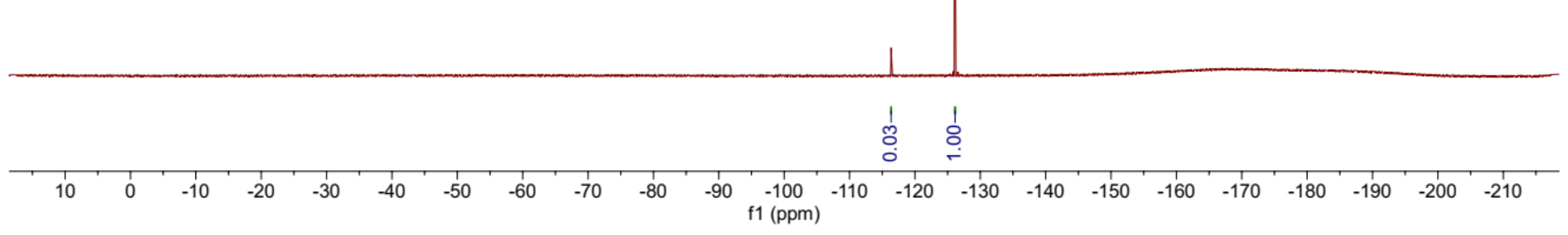




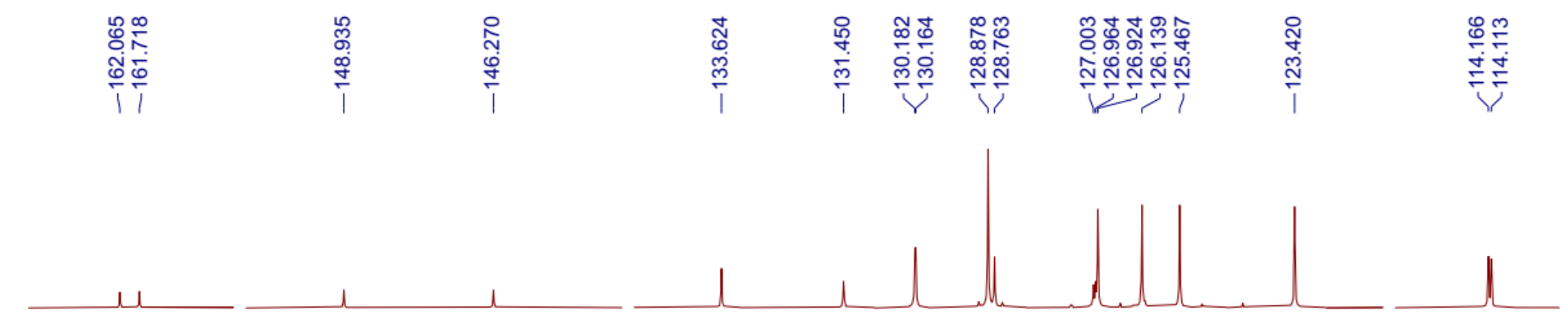

$\begin{array}{lllllllllllllllllllllllllllllllllllll}163 & 162 & 161 & 150 & 149 & 148 & 147 & 146 & 145 & 14435 & 134 & 133 & 132 & 131 & 130 & 129 & 128 & 127 & 126 & 125 & 124 & 123 & 122 & 115 & 114 & 113\end{array}$ f1 (ppm)

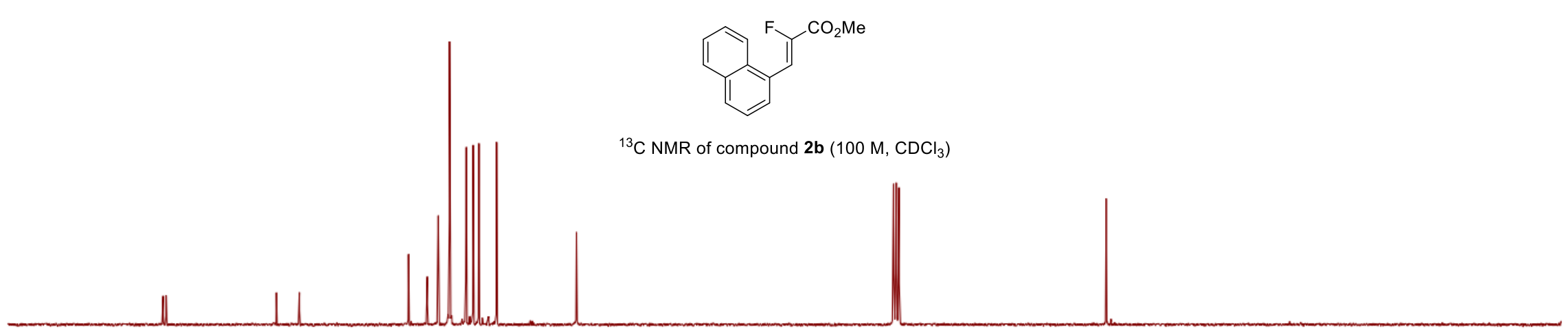

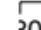

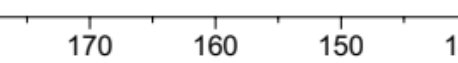

$140 \quad 130$

120

$110 \quad 100$

90

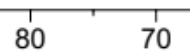

60

50

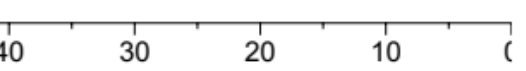




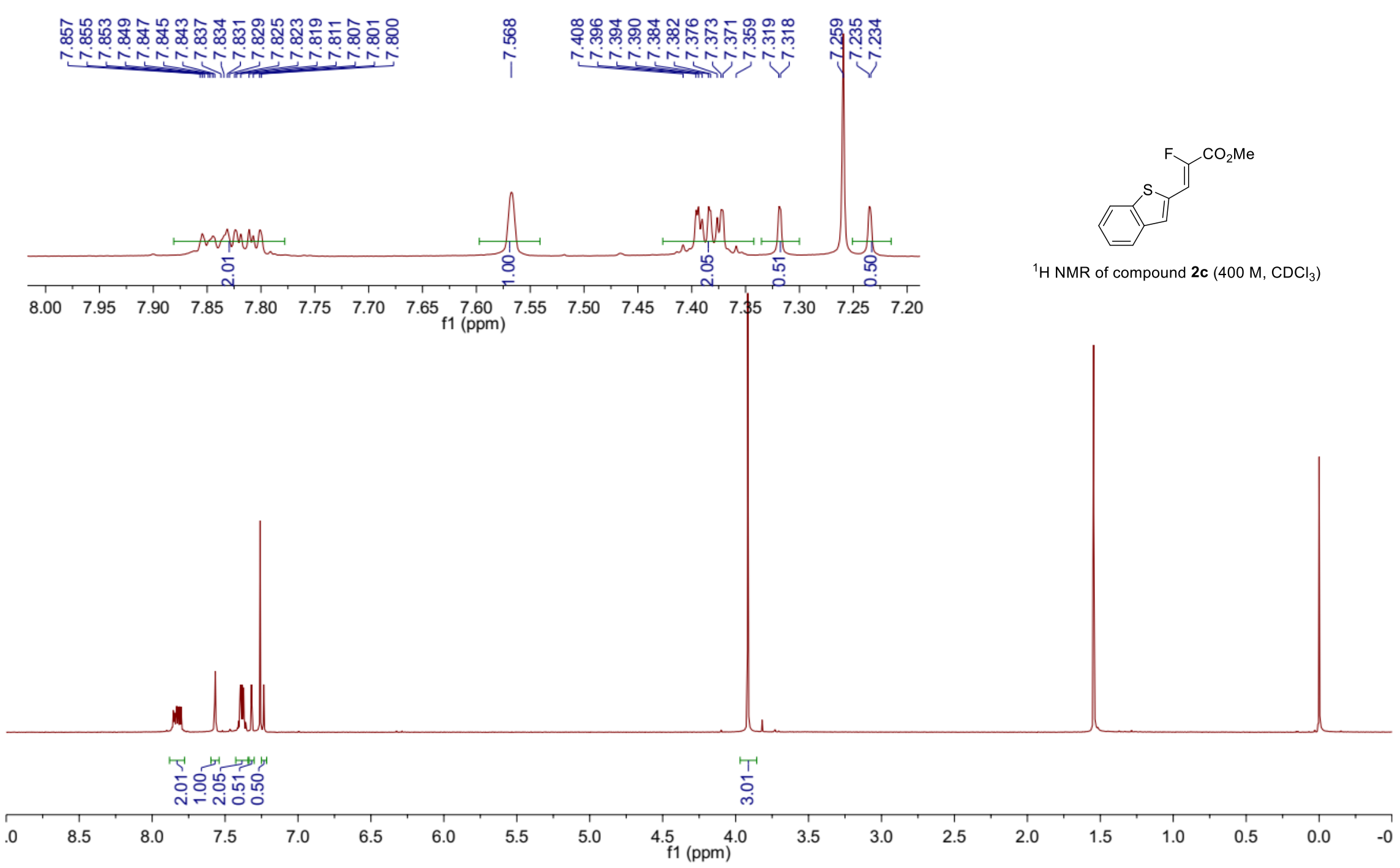


XSL-XI-81-XJ-30-400M. 52. fid

XSL-XI-81-XJ-30-400M F

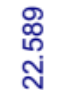

ลั่

${ }^{19} \mathrm{~F}$ NMR of compound $2 \mathrm{c}\left(376 \mathrm{M}, \mathrm{CDCl}_{3}\right)$

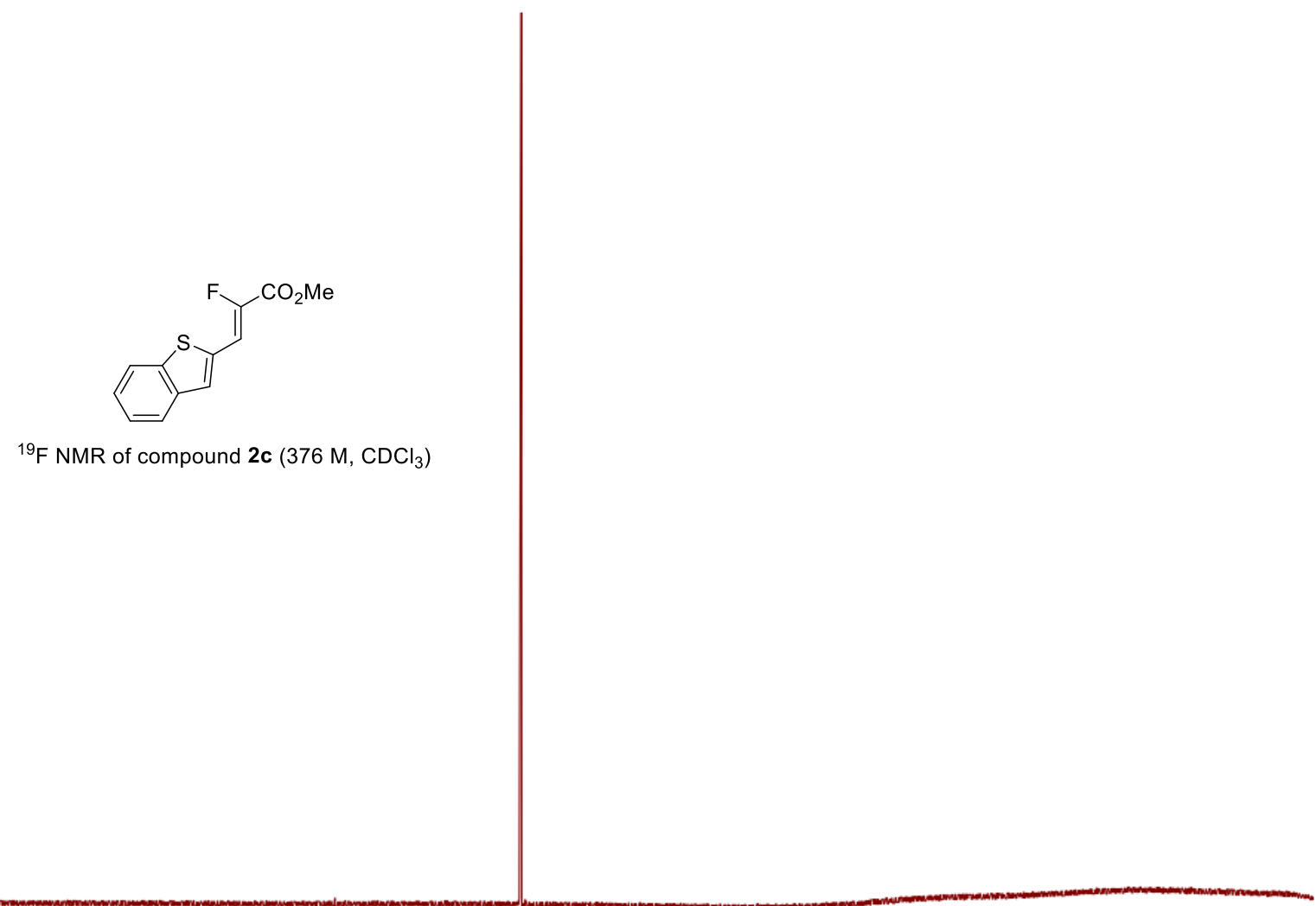

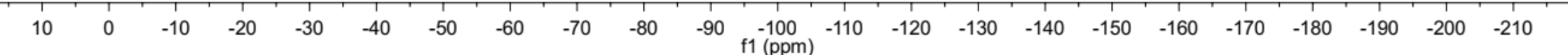




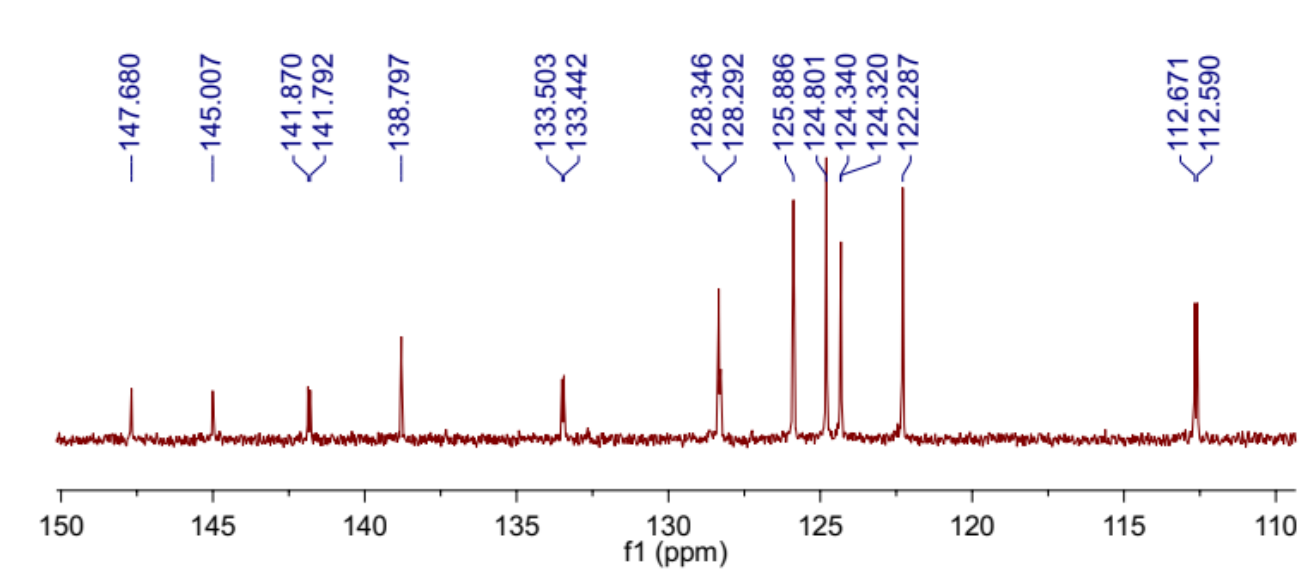

${ }^{13} \mathrm{C}$ NMR of compound $2 \mathrm{c}\left(100 \mathrm{M}, \mathrm{CDCl}_{3}\right)$
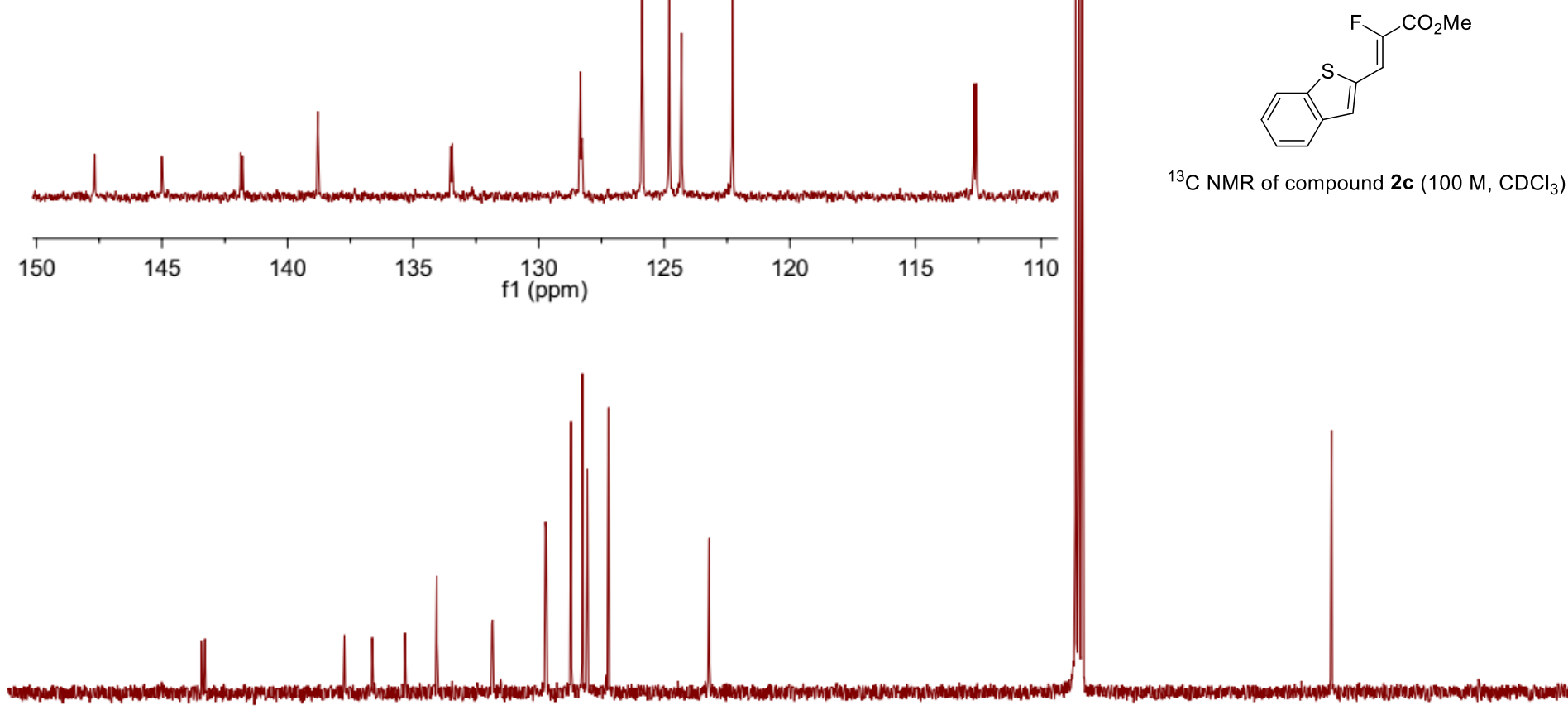

150

140

130

120

110

100

$\stackrel{90}{\mathrm{f} 1}(\mathrm{ppm})$

80

70

60

$50 \quad 40$

30 


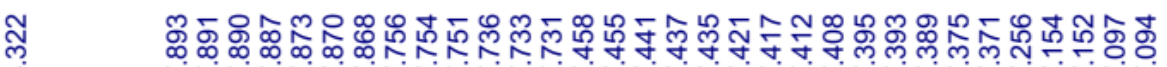

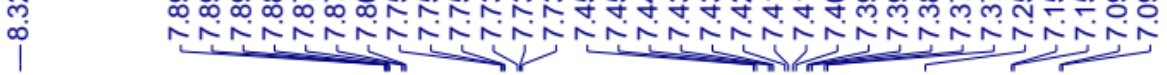

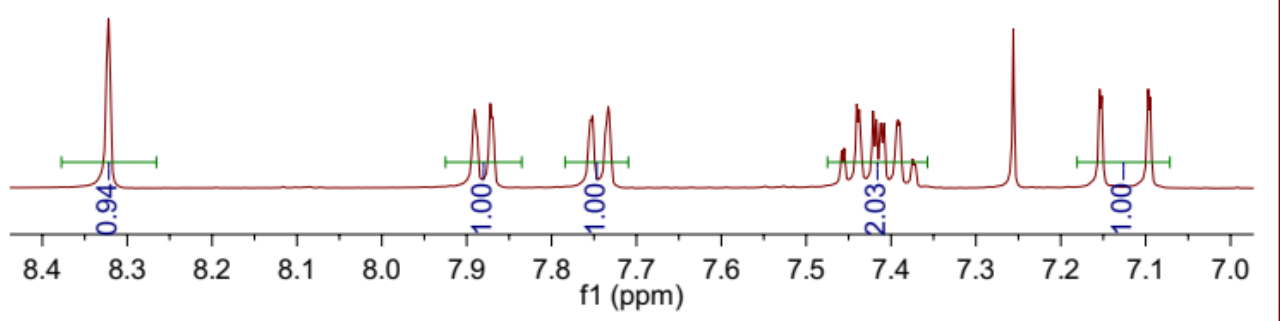

${ }^{1} \mathrm{H}$ NMR of compound (E)-2d $\left(400 \mathrm{M}, \mathrm{CDCl}_{3}\right)$
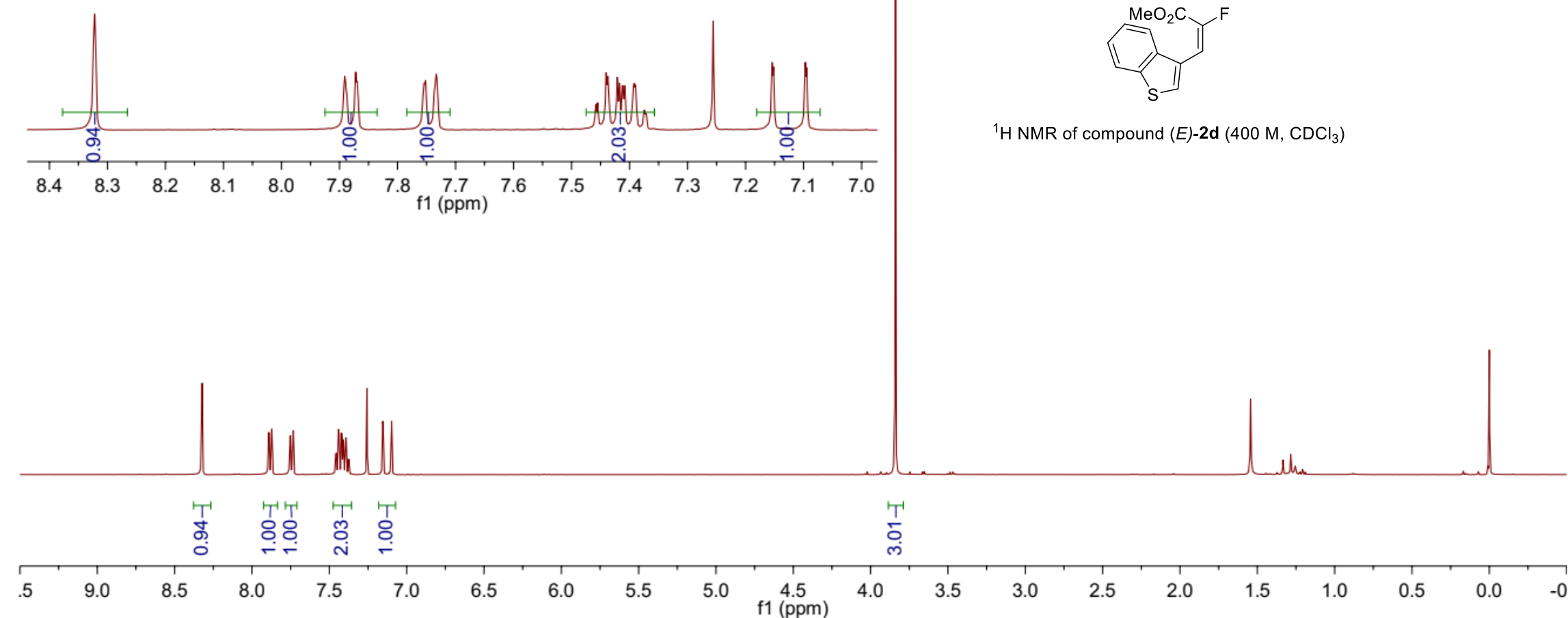
XSL-XJ-69-P1-400M-2. 42. fid

XSL-XJ-69-P1-400M-2 F

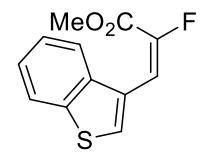

${ }^{19} \mathrm{~F} \mathrm{NMR}$ of compound $(E)-2 \mathbf{d}\left(376 \mathrm{M}, \mathrm{CDCl}_{3}\right)$

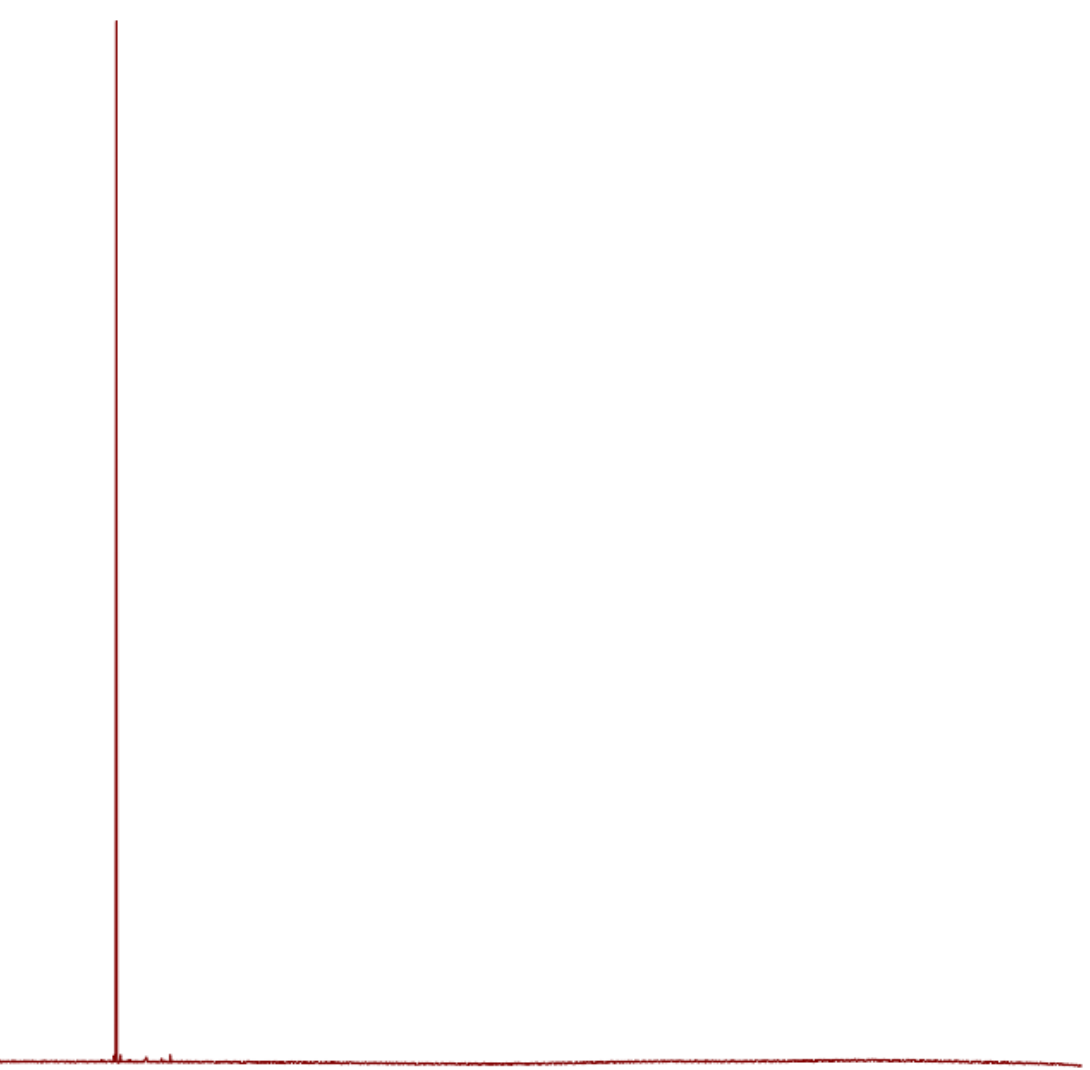

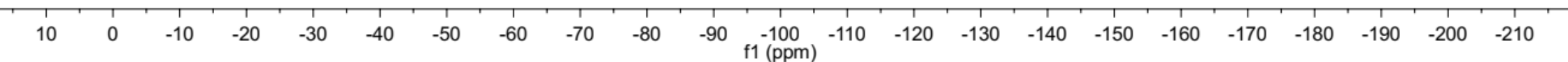


XSL-XJ-69-P1-400њCतN 0. fid

XSL-XJ-69-P1-400सेत

ర్థ

幽过

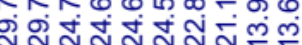

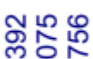

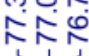

ঙ্ల్ల
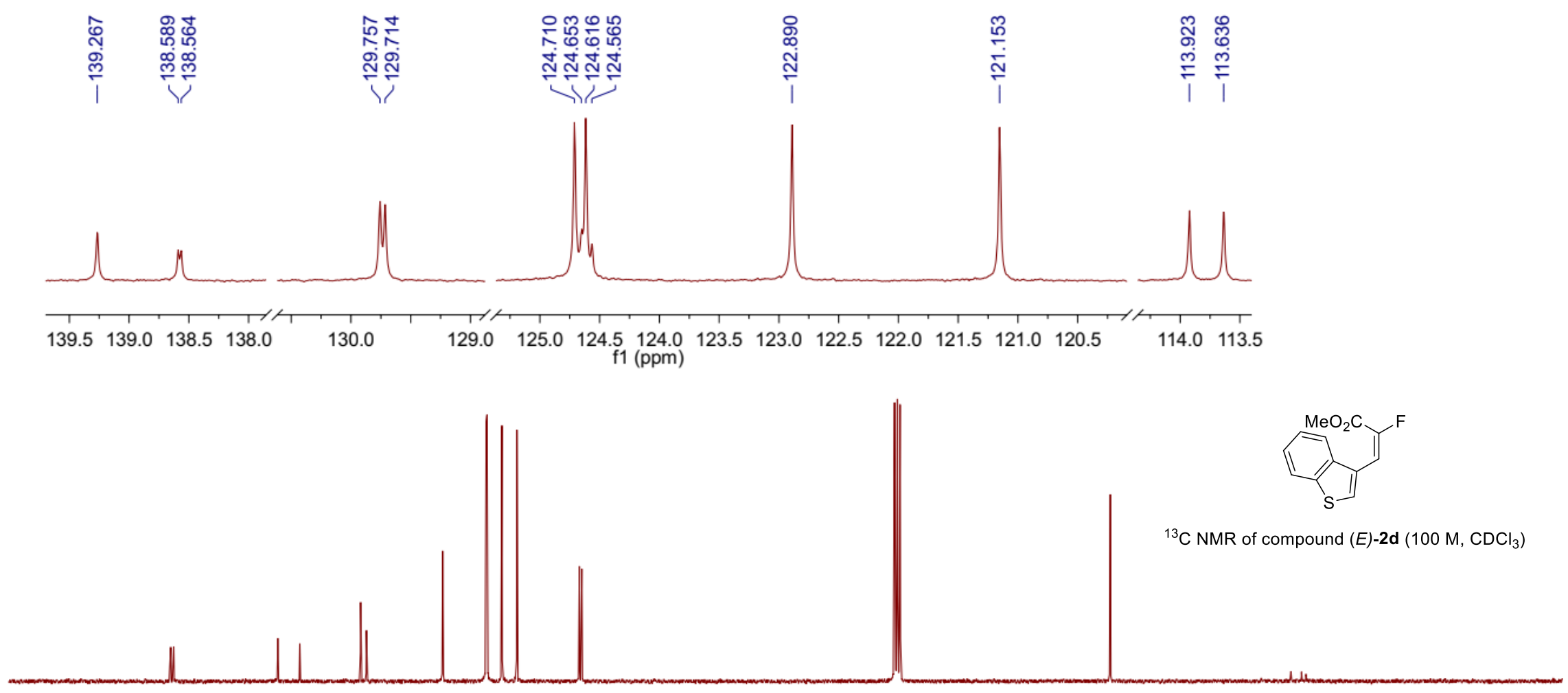

$$
30
$$

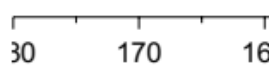

$50 \quad 140$

130

120

110

100

f1 $(\mathrm{ppm})$

1
80

$1, \quad 1$

40

$30 \quad 20 \quad 10 \div$




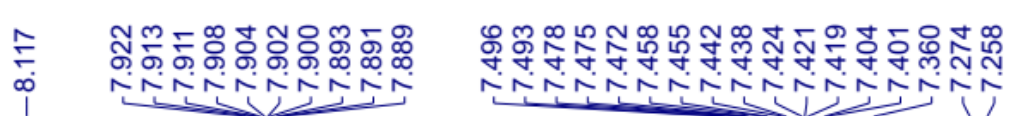
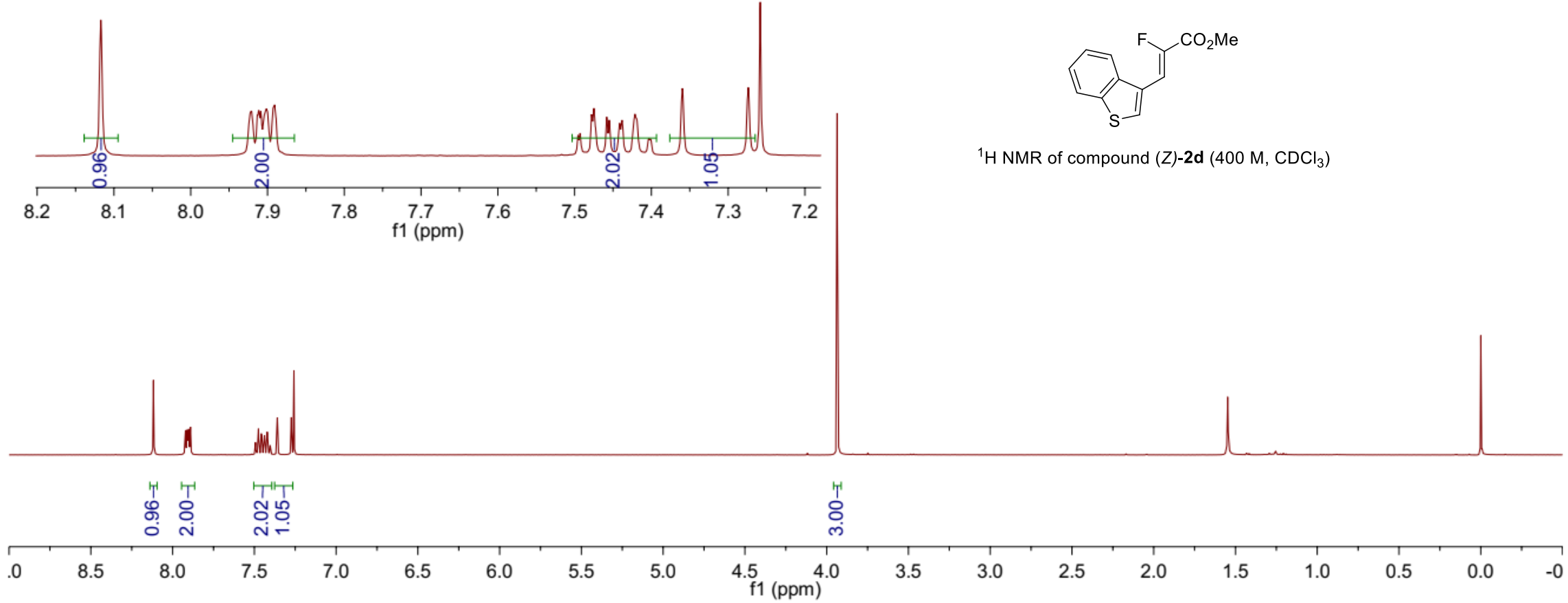
XSL-XJ-69-P2-400M-2. 52. fid

XSL-XJ-69-P2-400M-2 F

${ }^{19} \mathrm{~F}$ NMR of compound (Z)-2d $\left(376 \mathrm{M}, \mathrm{CDCl}_{3}\right)$
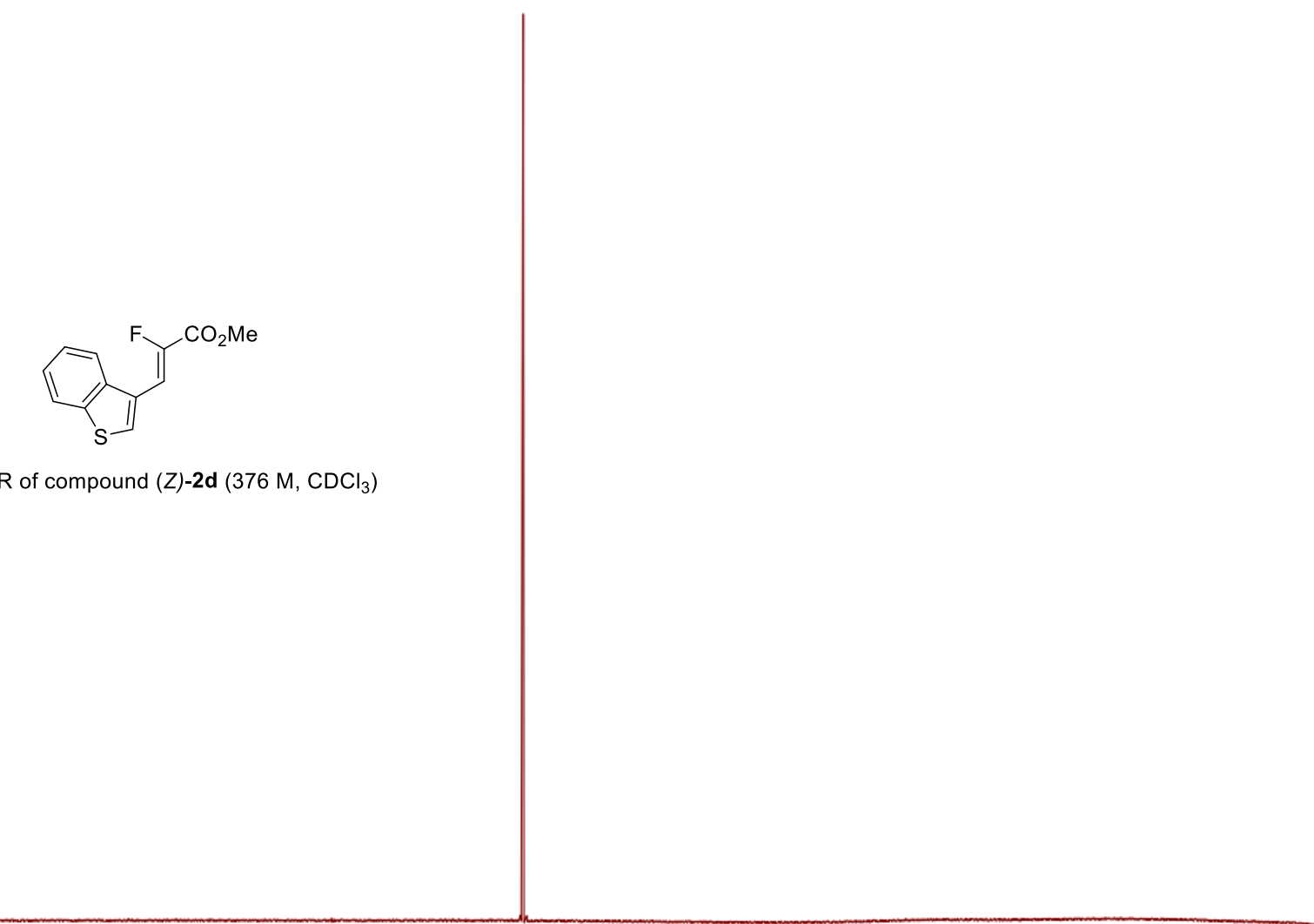
XSL-XJ-69-P2-400MC. 10. fid XSL-XJ-69-P2-400MC

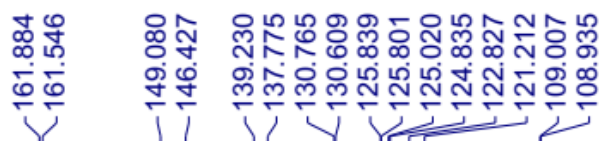

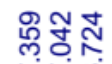

চ্口:

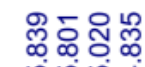

งกำ

추 촉

$\underset{\substack{n \\ \stackrel{N}{N}}}{\stackrel{i}{n}}$
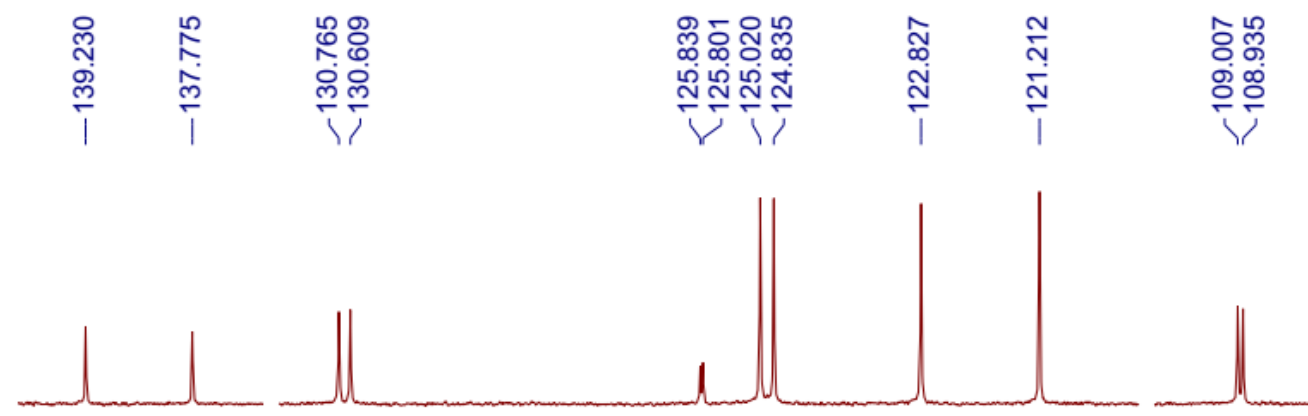

$\begin{array}{lllll}140 & 139 & 138 & 137 & 131\end{array}$
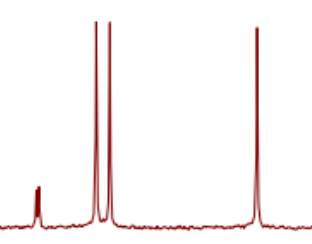

(1)

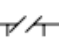

$\begin{array}{lllllllllllllllll}140 & 139 & 138 & 137 & 131 & 130 & 129 & 128 & 127 & 126 & 125 & 124 & 123 & 122 & 121 & 120110 & 108\end{array}$

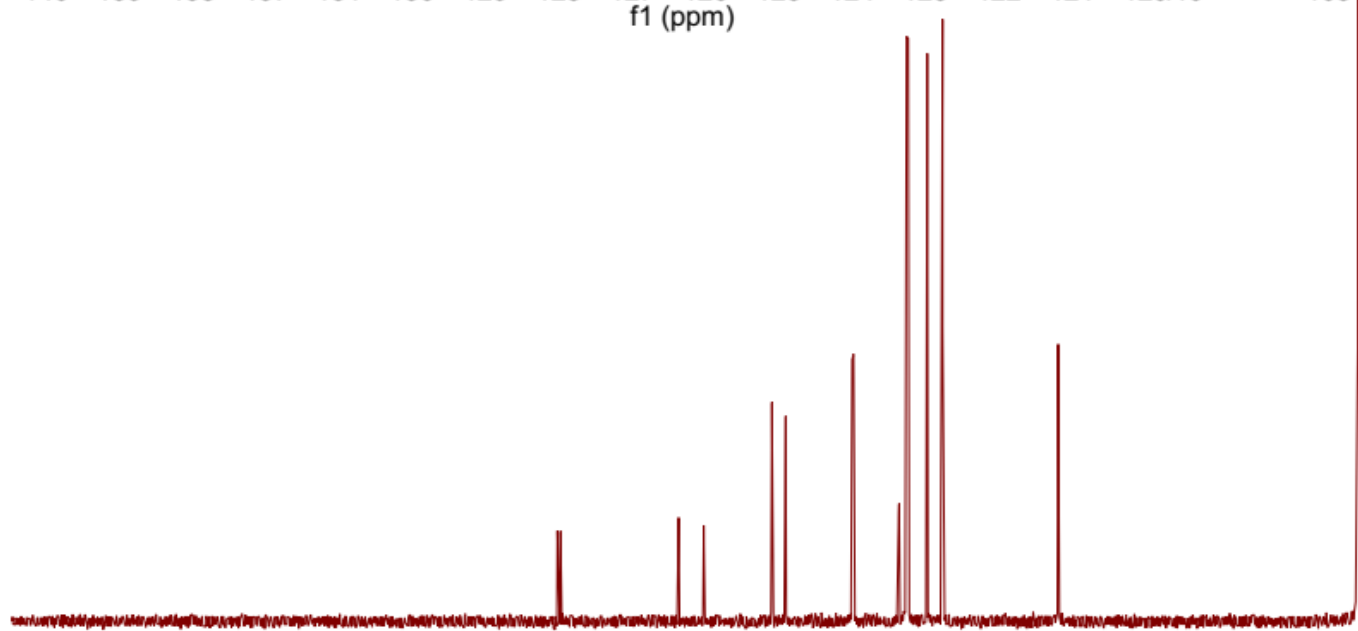

$210 \quad 200$ 


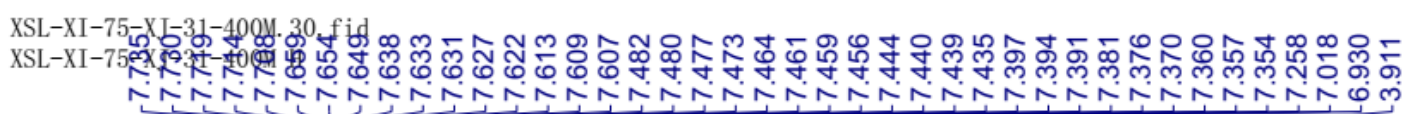

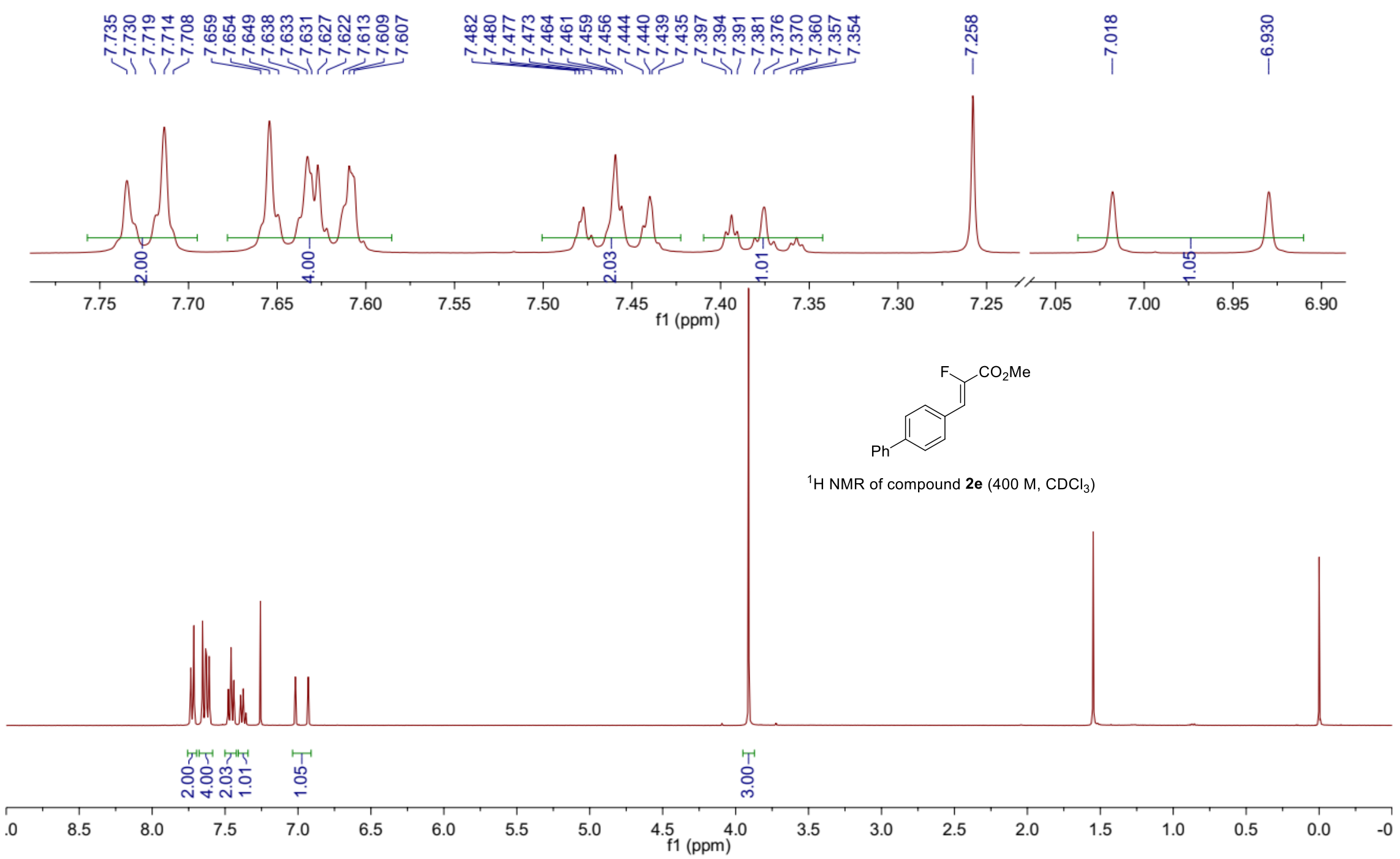


XSL-XI-75-XJ-31-400M. 32. fid

XSL-XI-75-XJ-31-400M F

$\frac{\stackrel{\infty}{+}}{\stackrel{+}{\mathfrak{i}}}$

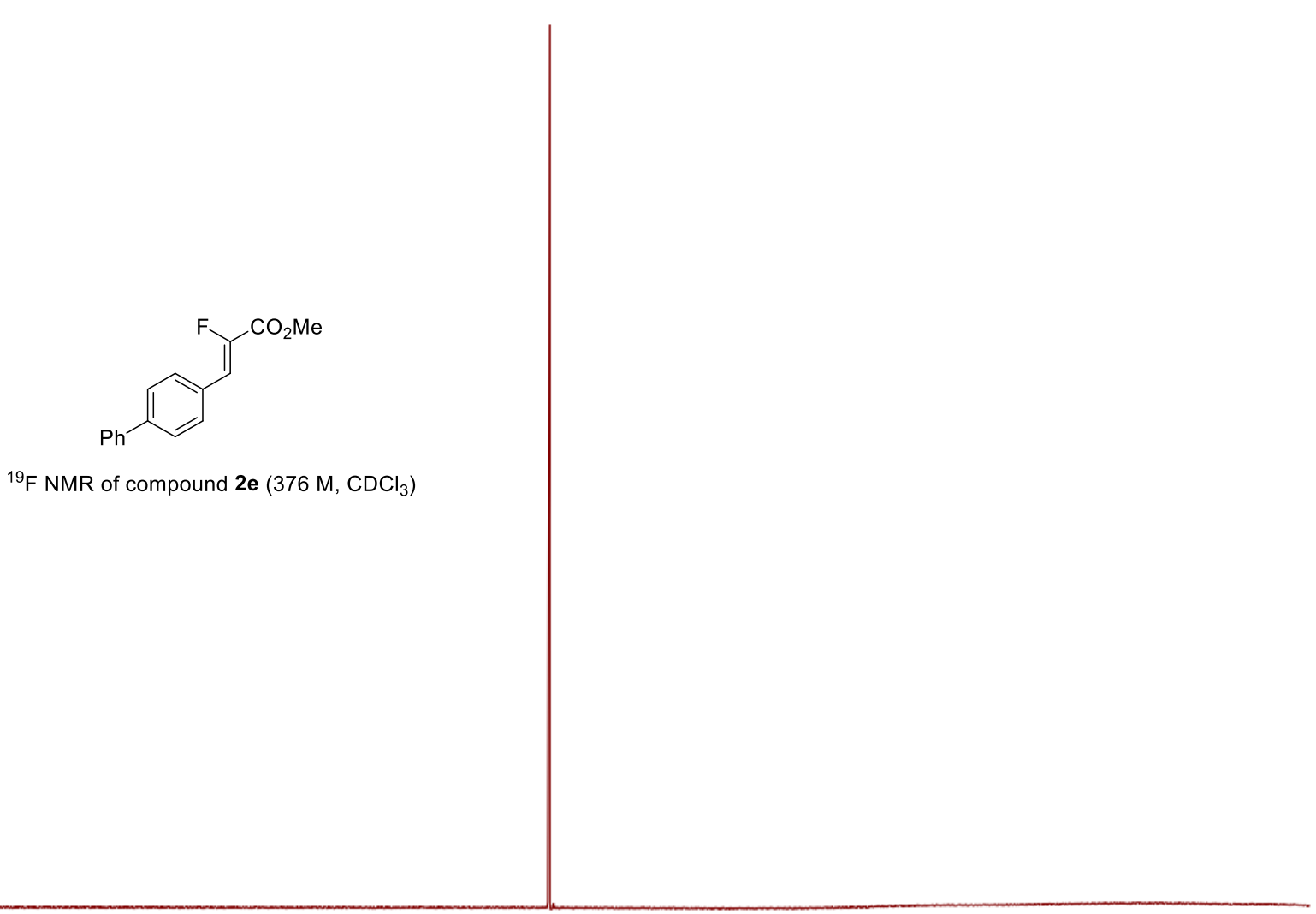

${ }^{19} \mathrm{~F} \mathrm{NMR}$ of compound $2 \mathrm{e}\left(376 \mathrm{M}, \mathrm{CDCl}_{3}\right)$ 


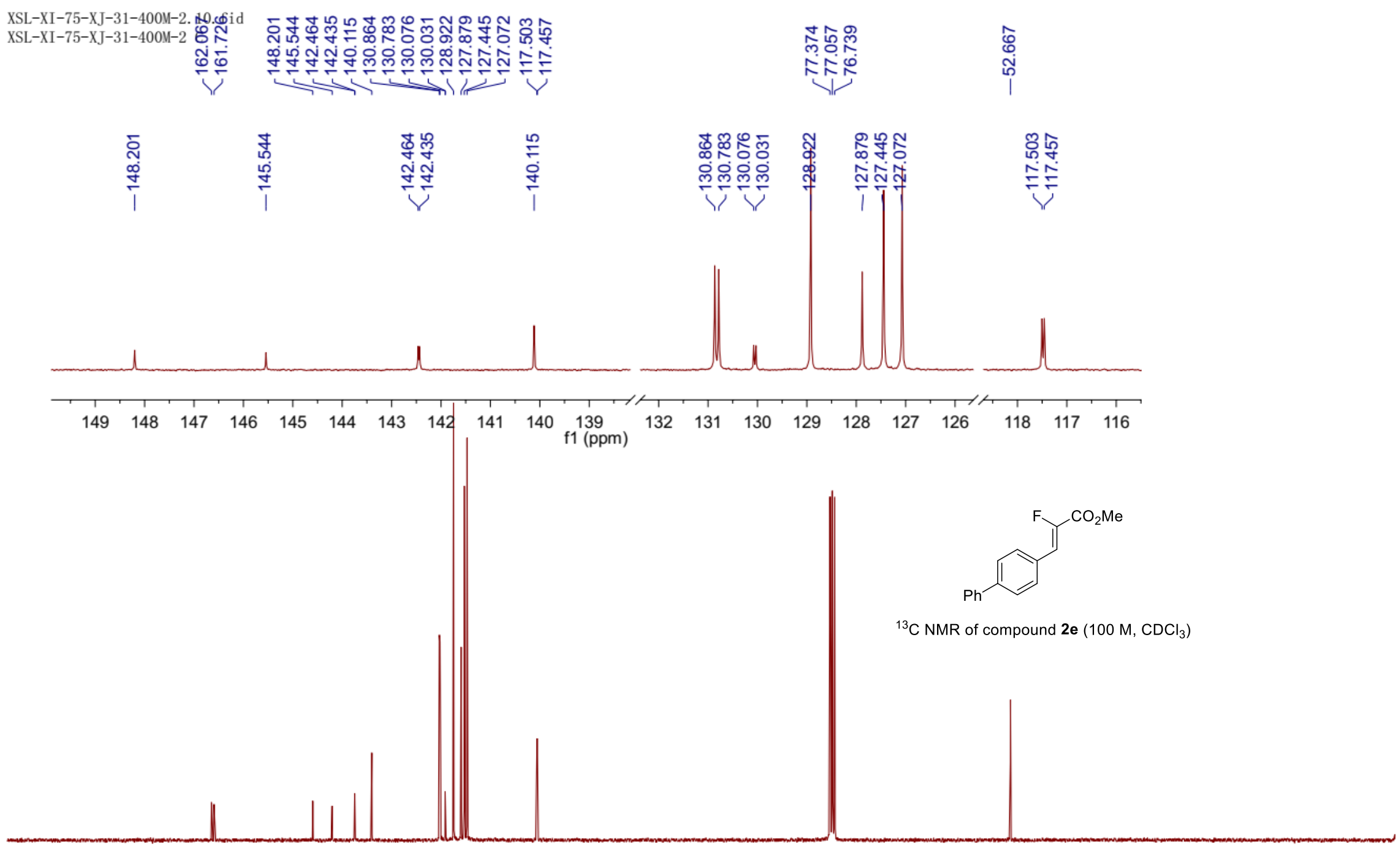

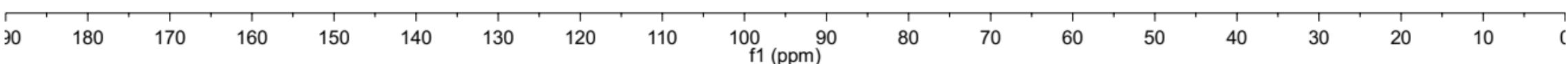




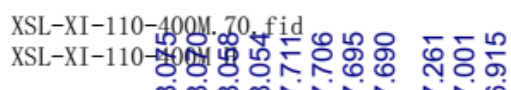

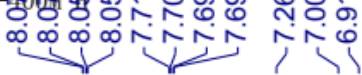

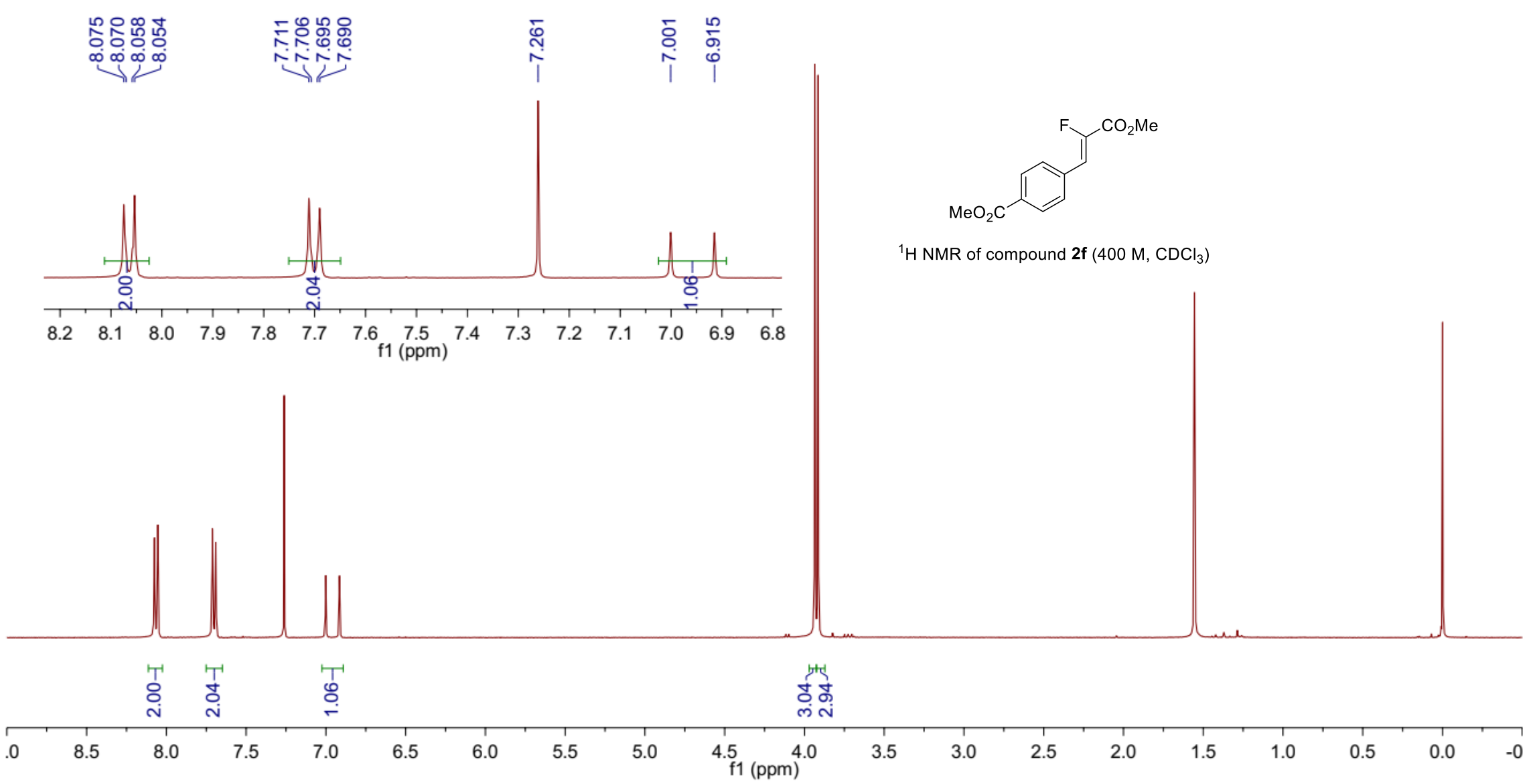


XSL-XI-110-400M. 72. fid

XSL-XI-110-400M F

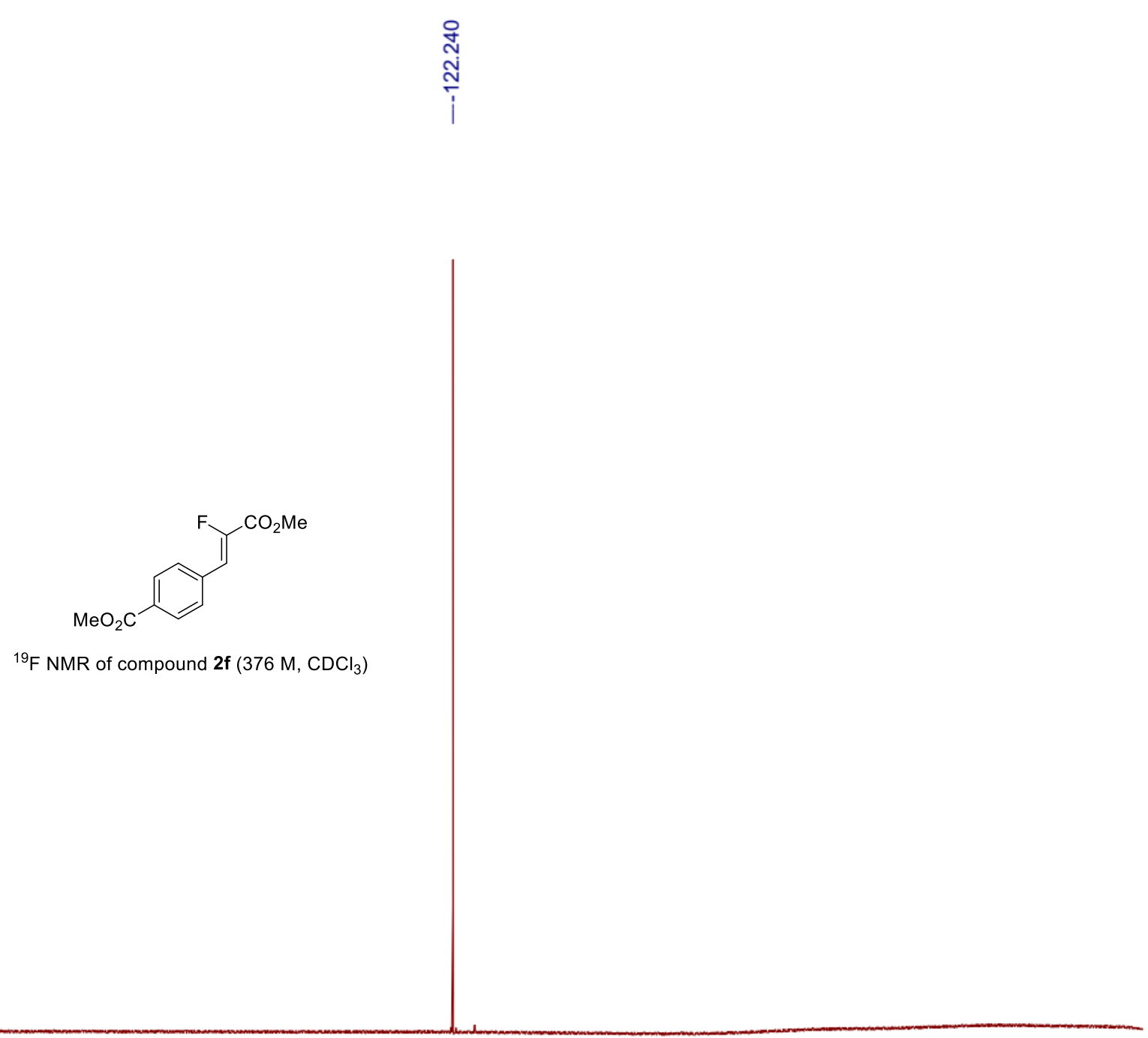

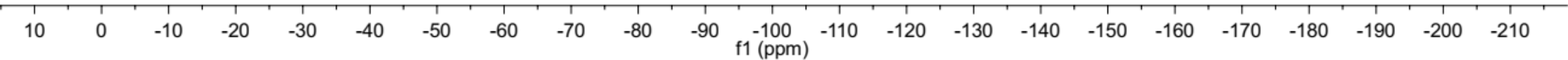




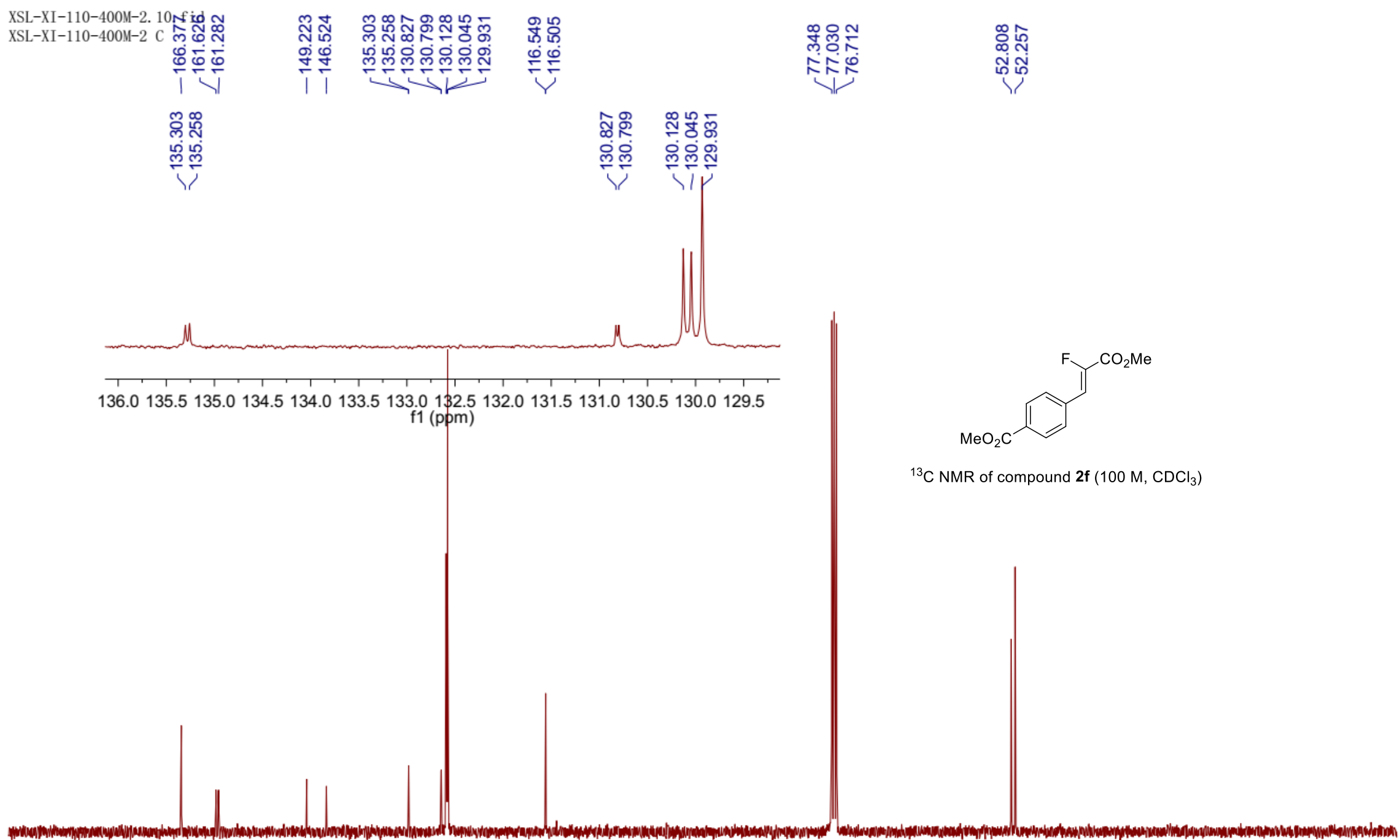

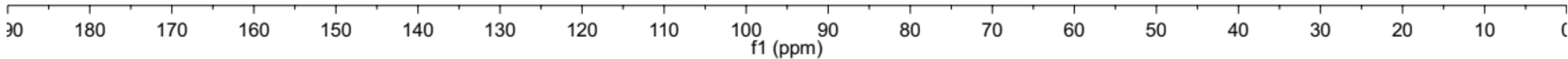




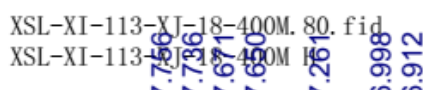

氷

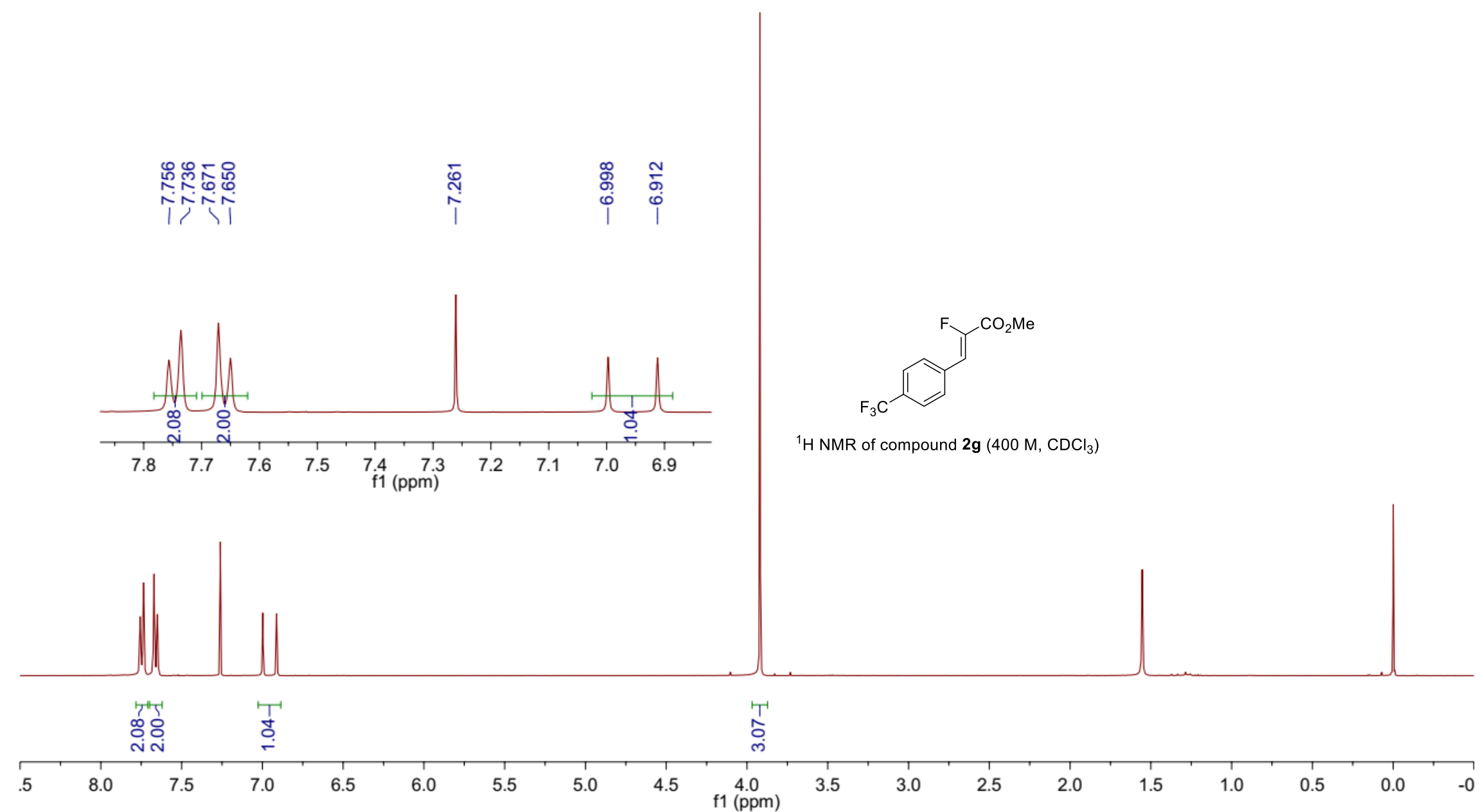


XSL-XI-113-XJ-18-400M. 82. fid

XSL-XI-113-XJ-18-400M F

\} $\\{\text { ஸ़ }} \\{\text { | }} \end{array}$

্ֻণ

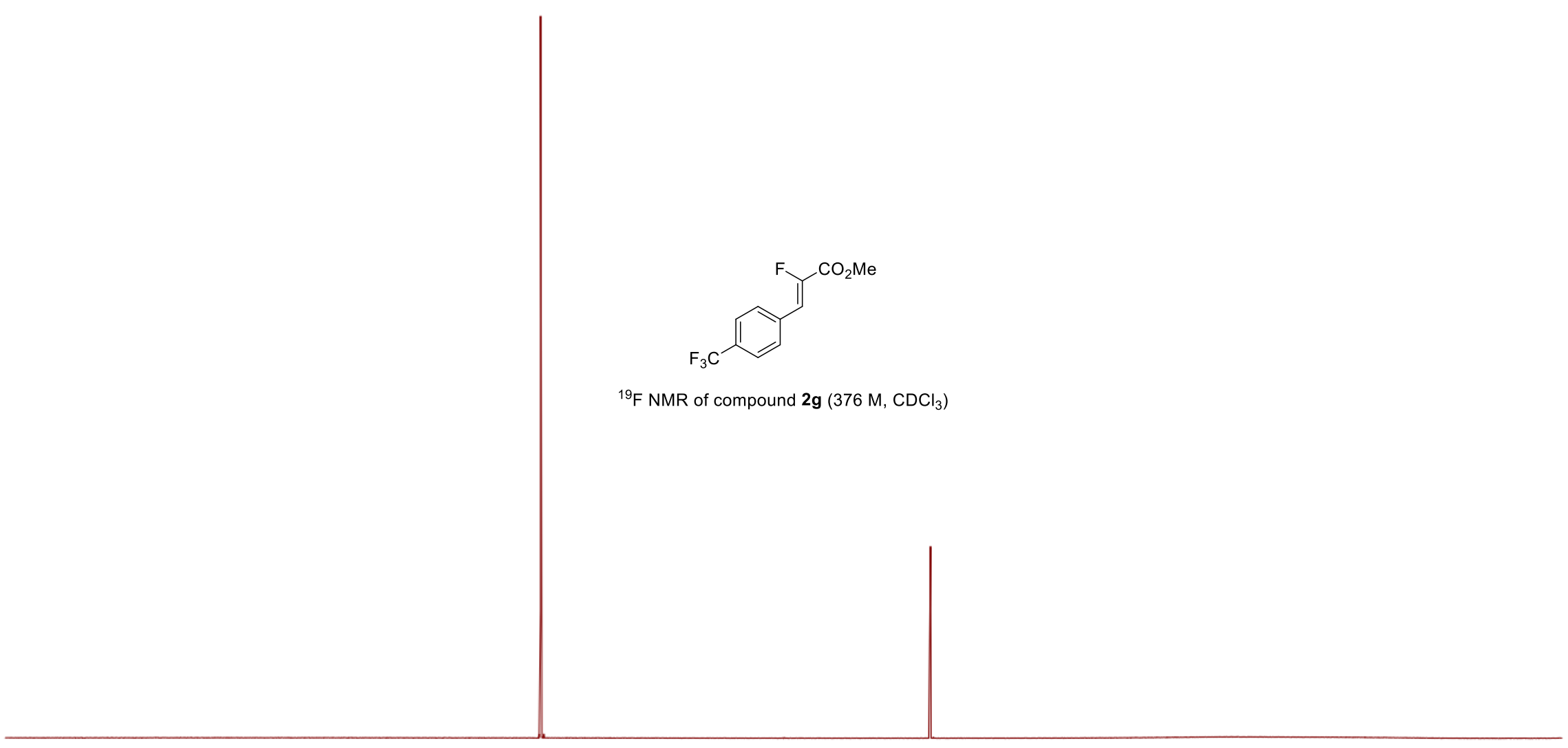




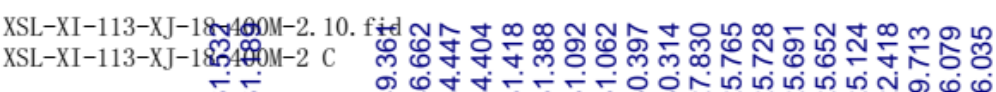

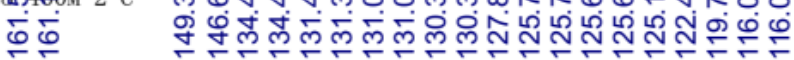

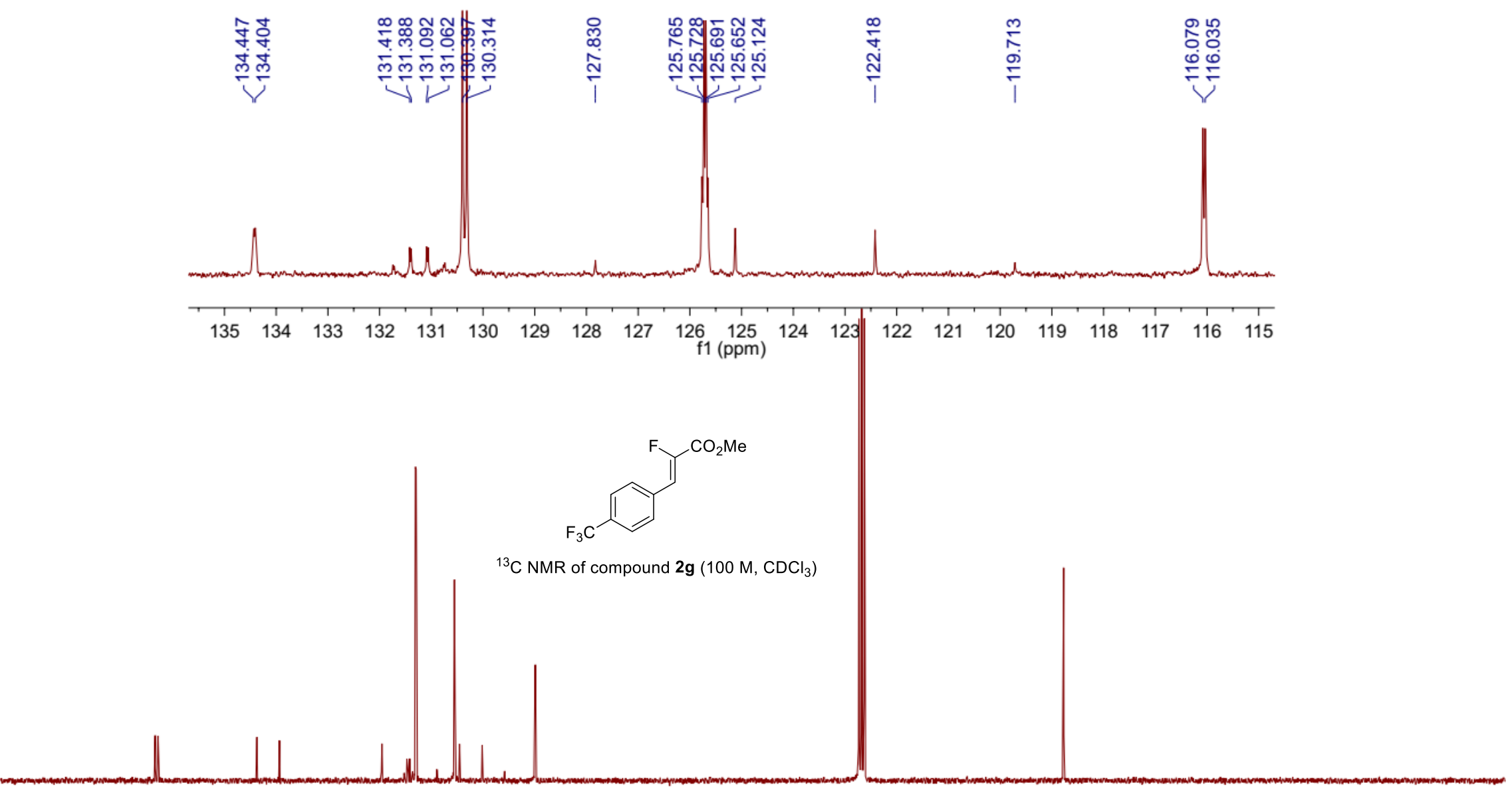

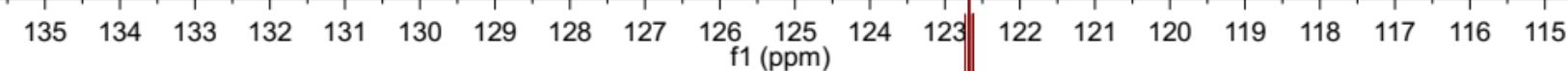

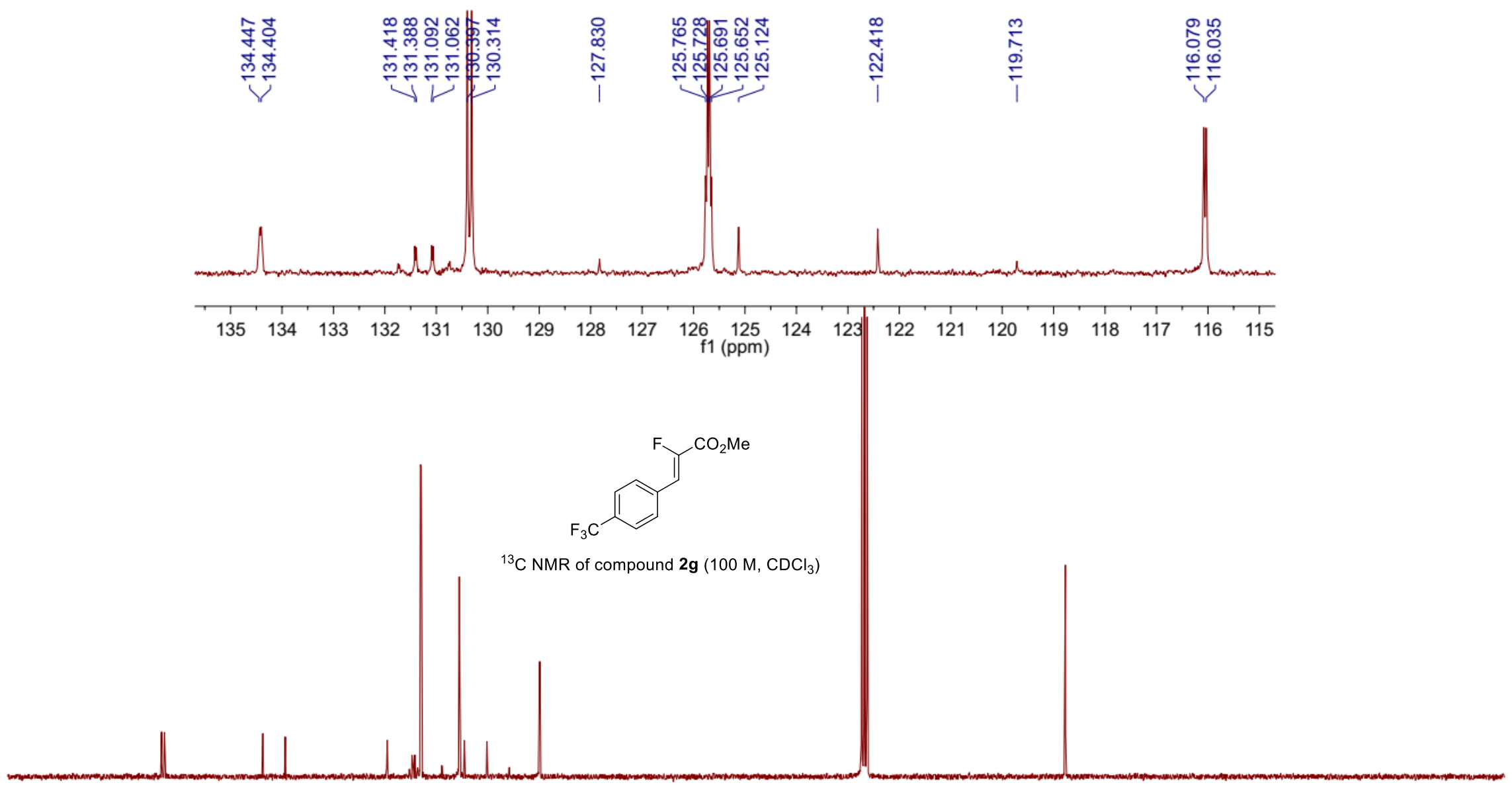

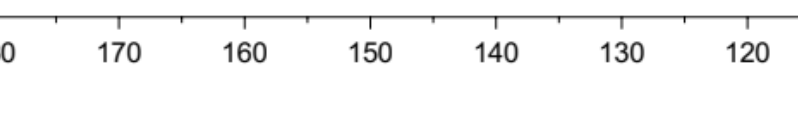

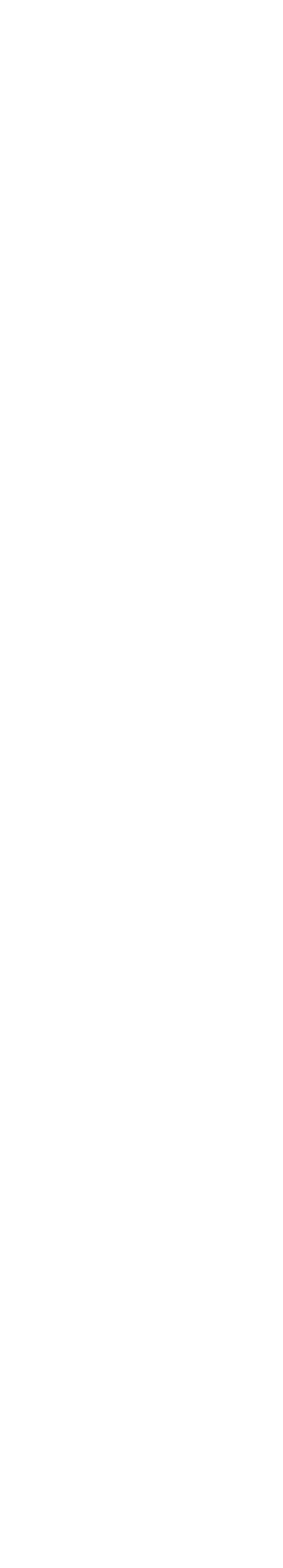
(n) 


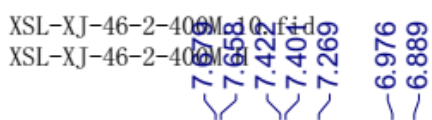

仓े

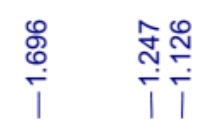

8
○ं
i

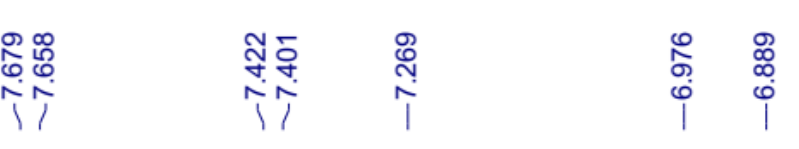
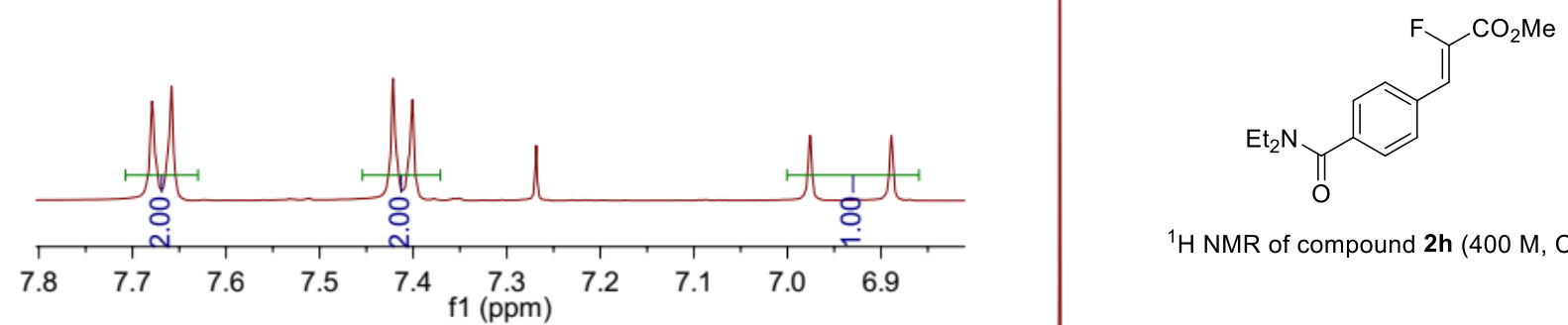

${ }^{1} \mathrm{H}$ NMR of compound $2 \mathrm{~h}\left(400 \mathrm{M}, \mathrm{CDCl}_{3}\right)$
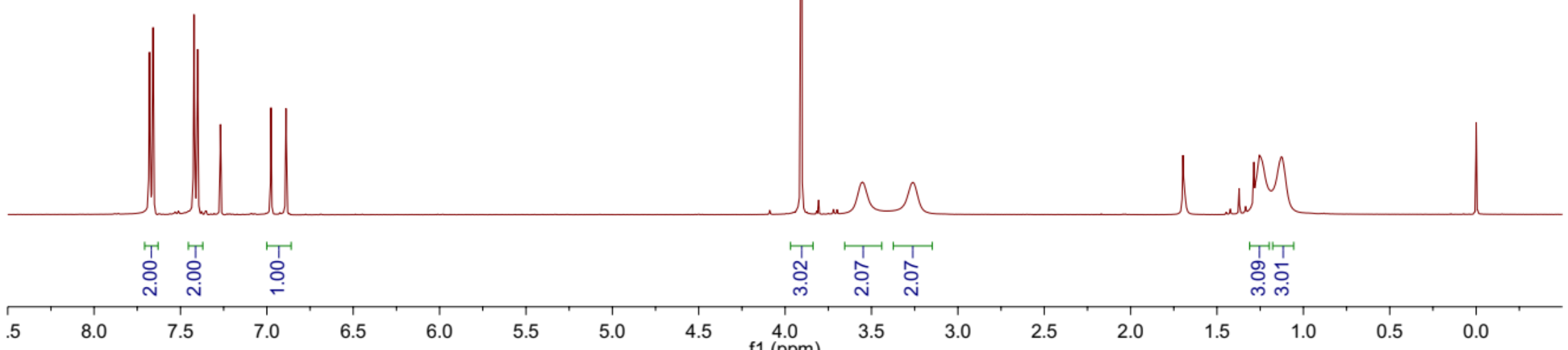

ชิ

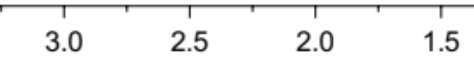

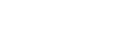


XSL-XJ-46-2-400M. 11. fid

XSL-XJ-46-2-400M F

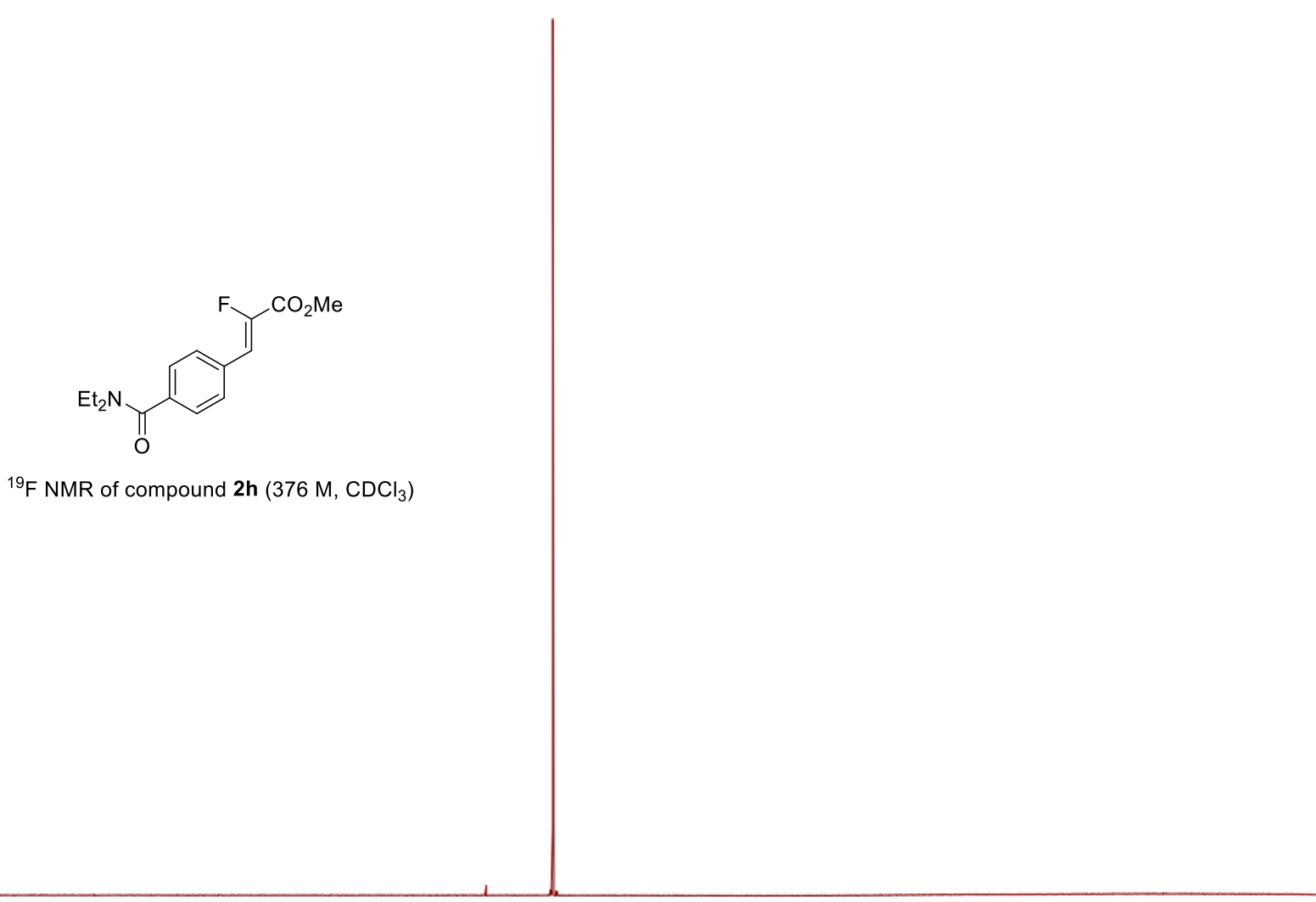


只

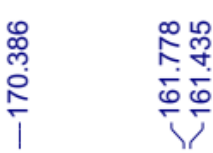

응 ల్ల

守 守

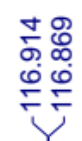

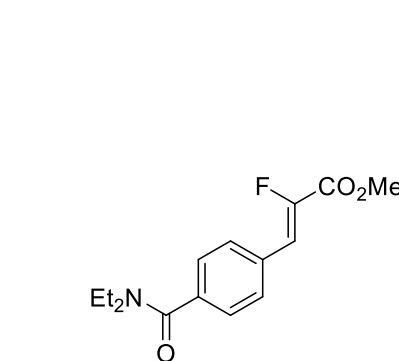

${ }^{13} \mathrm{C} \mathrm{NMR}$ of compound $\mathbf{2 h}\left(100 \mathrm{M}, \mathrm{CDCl}_{3}\right)$
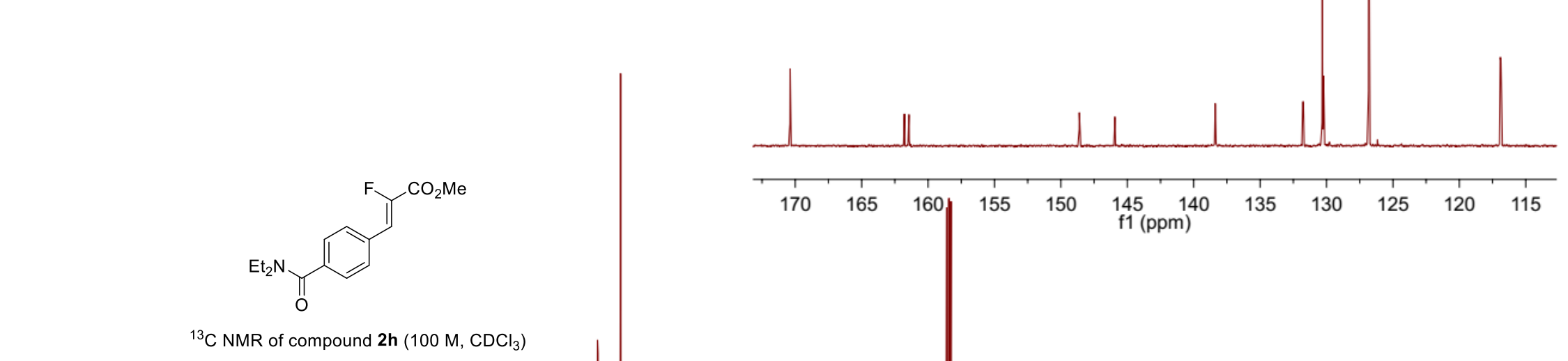

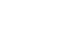

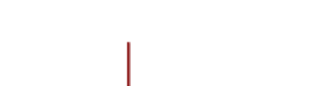




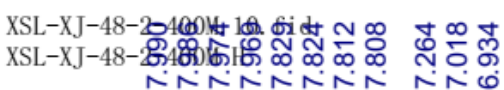

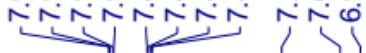

尽

$\frac{\hat{n}}{i}$

웅

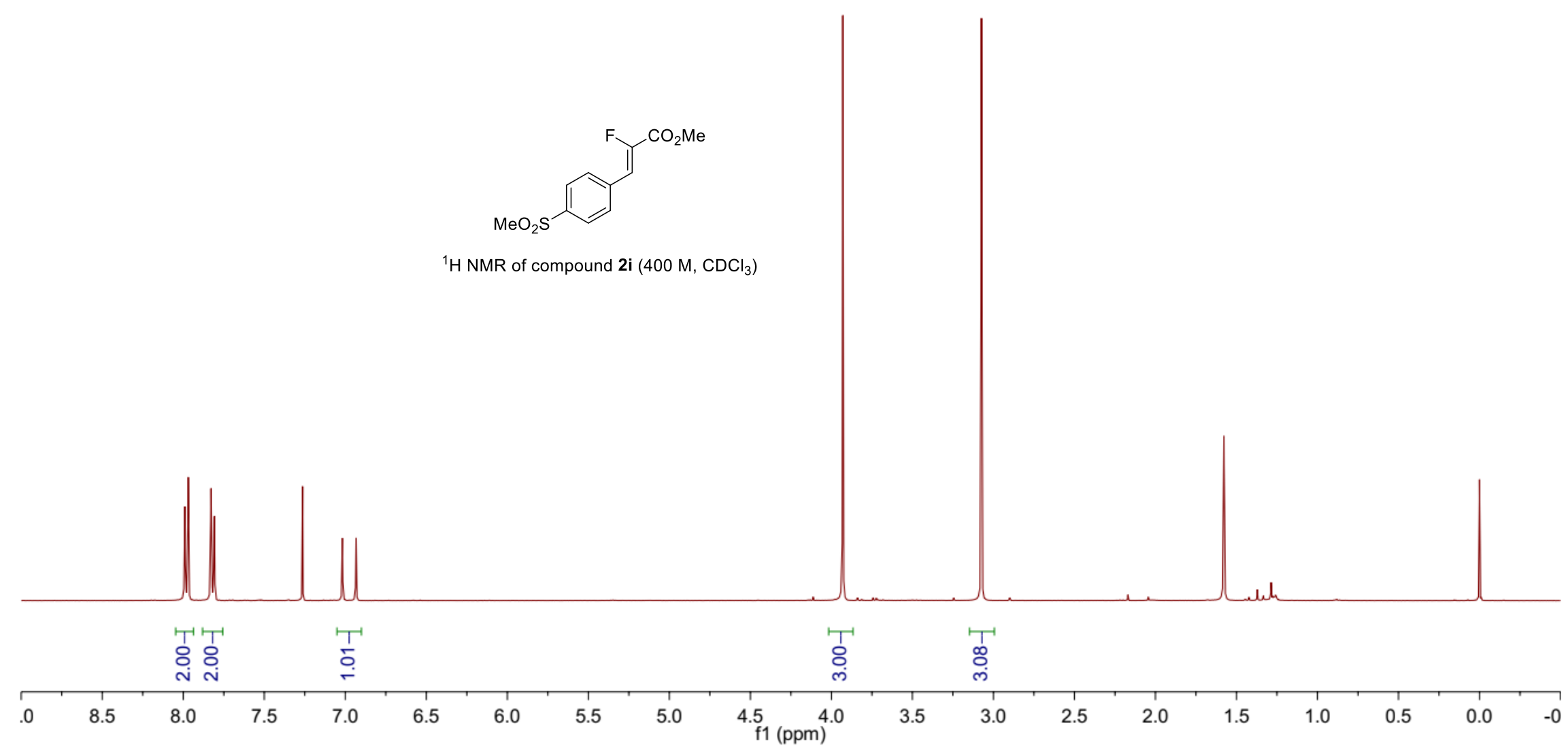


XSL-XJ-48-2-400M. 11. fid

XSL-XJ-48-2-400M F

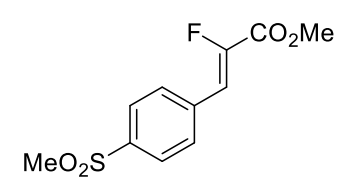

${ }^{19} \mathrm{~F} \mathrm{NMR}$ of compound $2 \mathbf{i}\left(376 \mathrm{M}, \mathrm{CDCl}_{3}\right)$ 

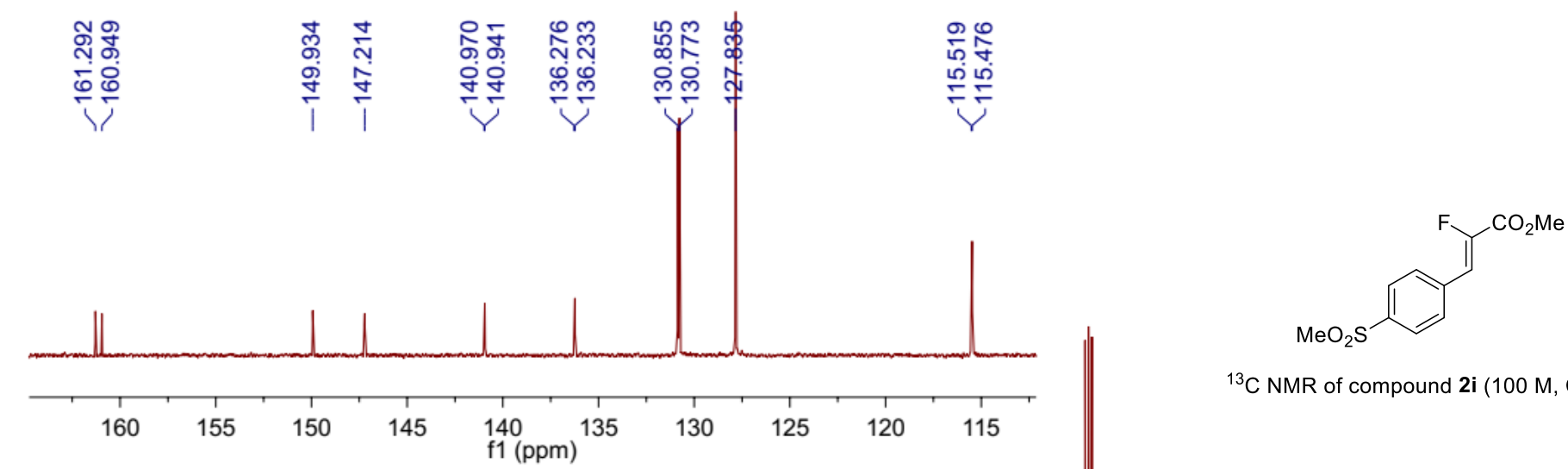

${ }^{13} \mathrm{C} \mathrm{NMR}$ of compound $2 \mathrm{i}\left(100 \mathrm{M}, \mathrm{CDCl}_{3}\right)$
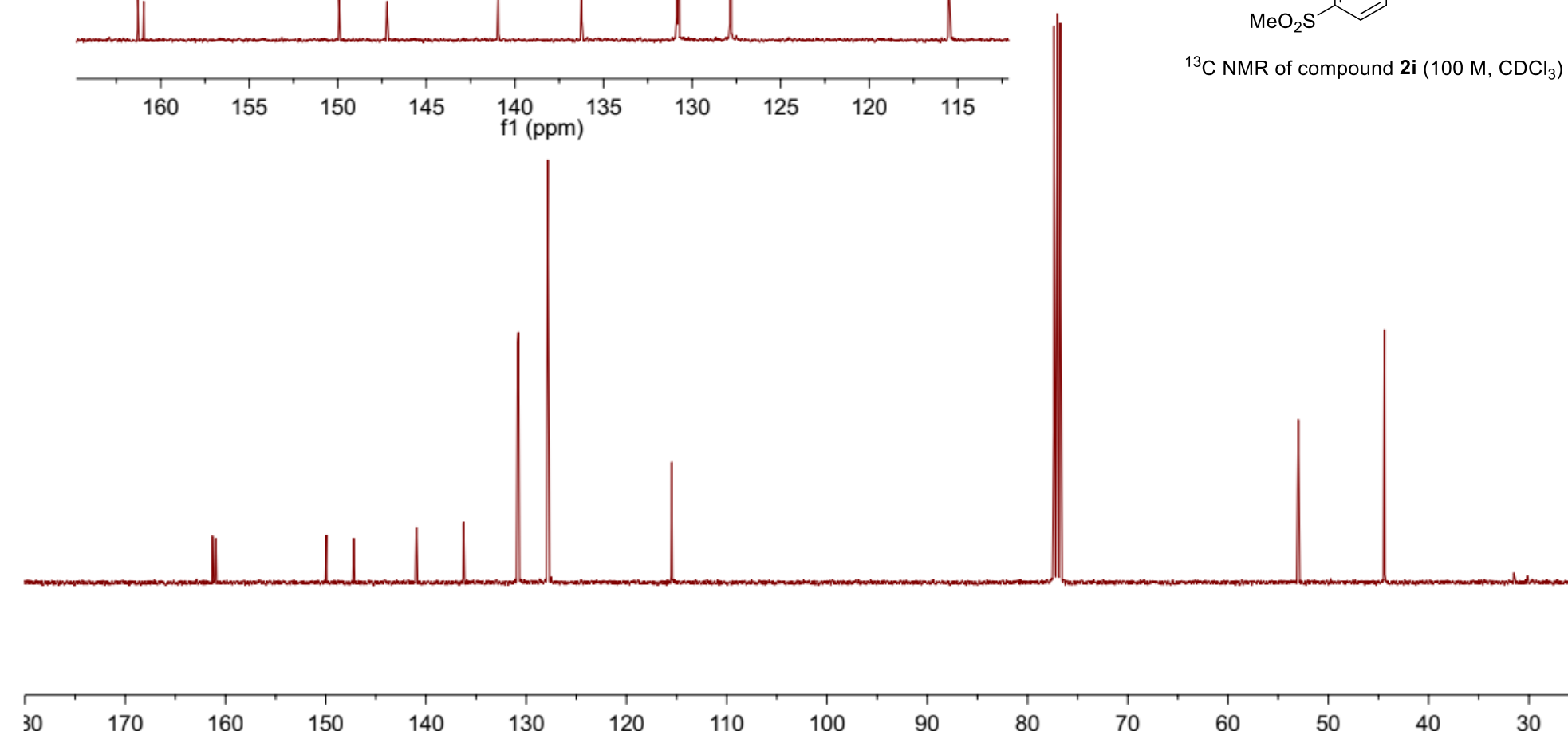

150 140 130 120 110 100 $\begin{array}{cc}1 & 1 \\ 90 & 80\end{array}$ 70 60 $50 \quad 40$ $\begin{array}{lll}1 & 1 \\ 30 & 20 & 10\end{array}$ 


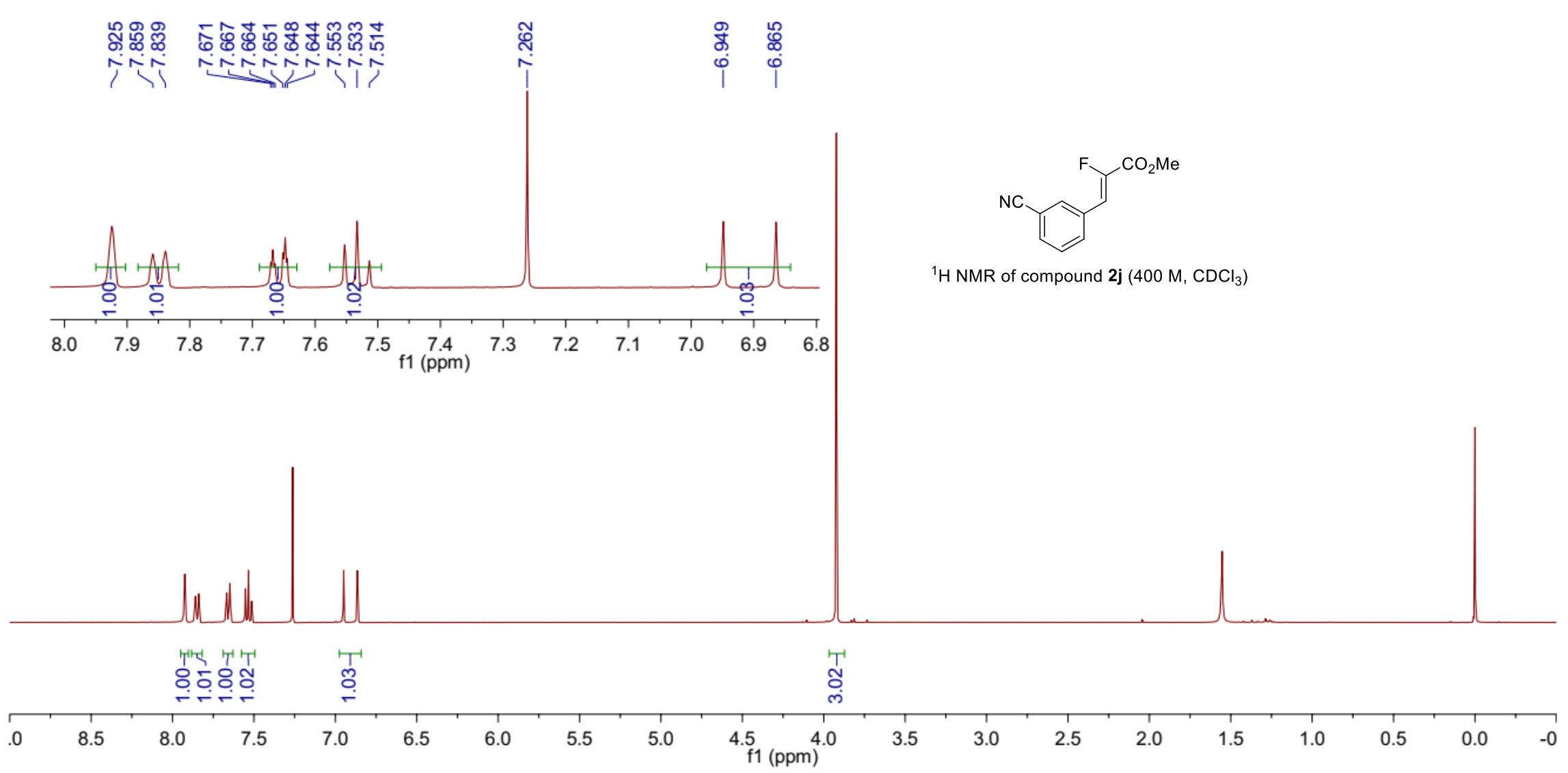


XSL-XI-61-400M. 22. fid

XSL-XI-61-400M F

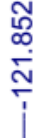

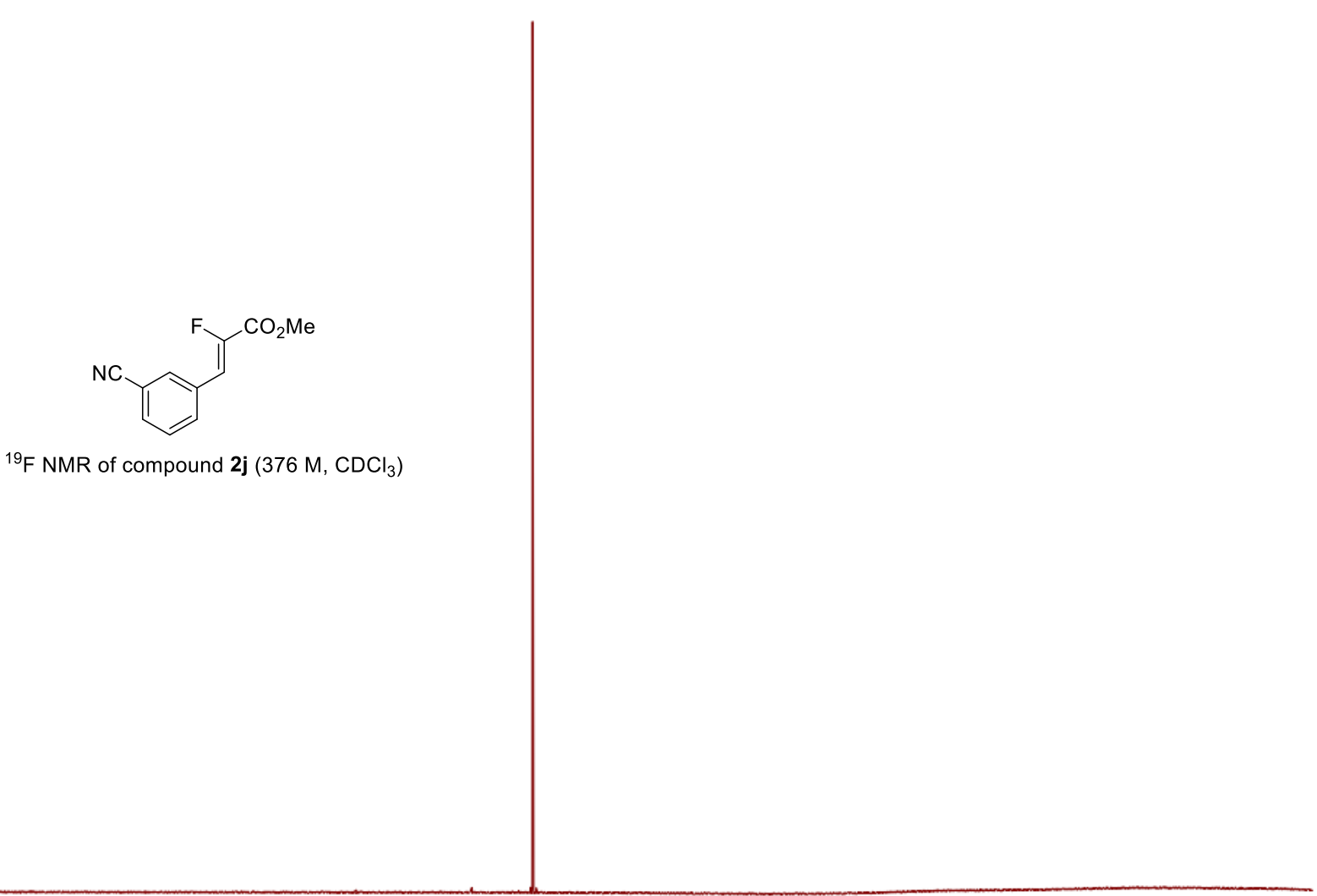

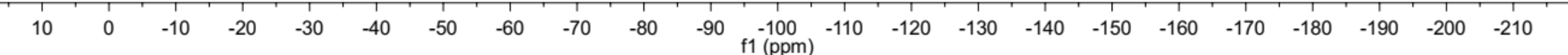




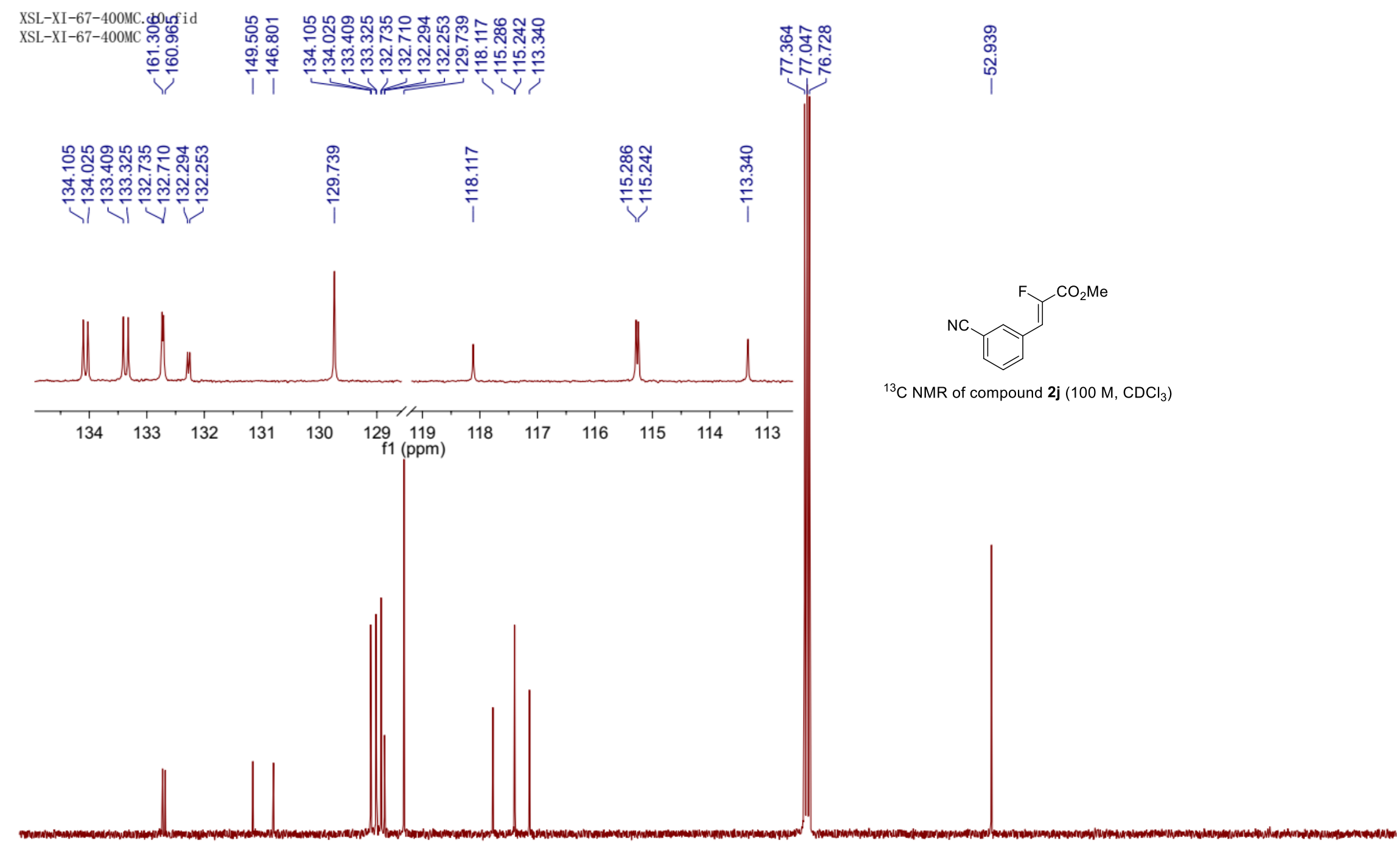

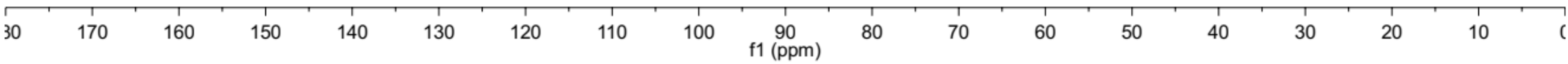




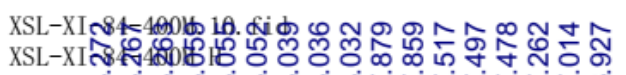

$\underbrace{0.000}_{0,000}$

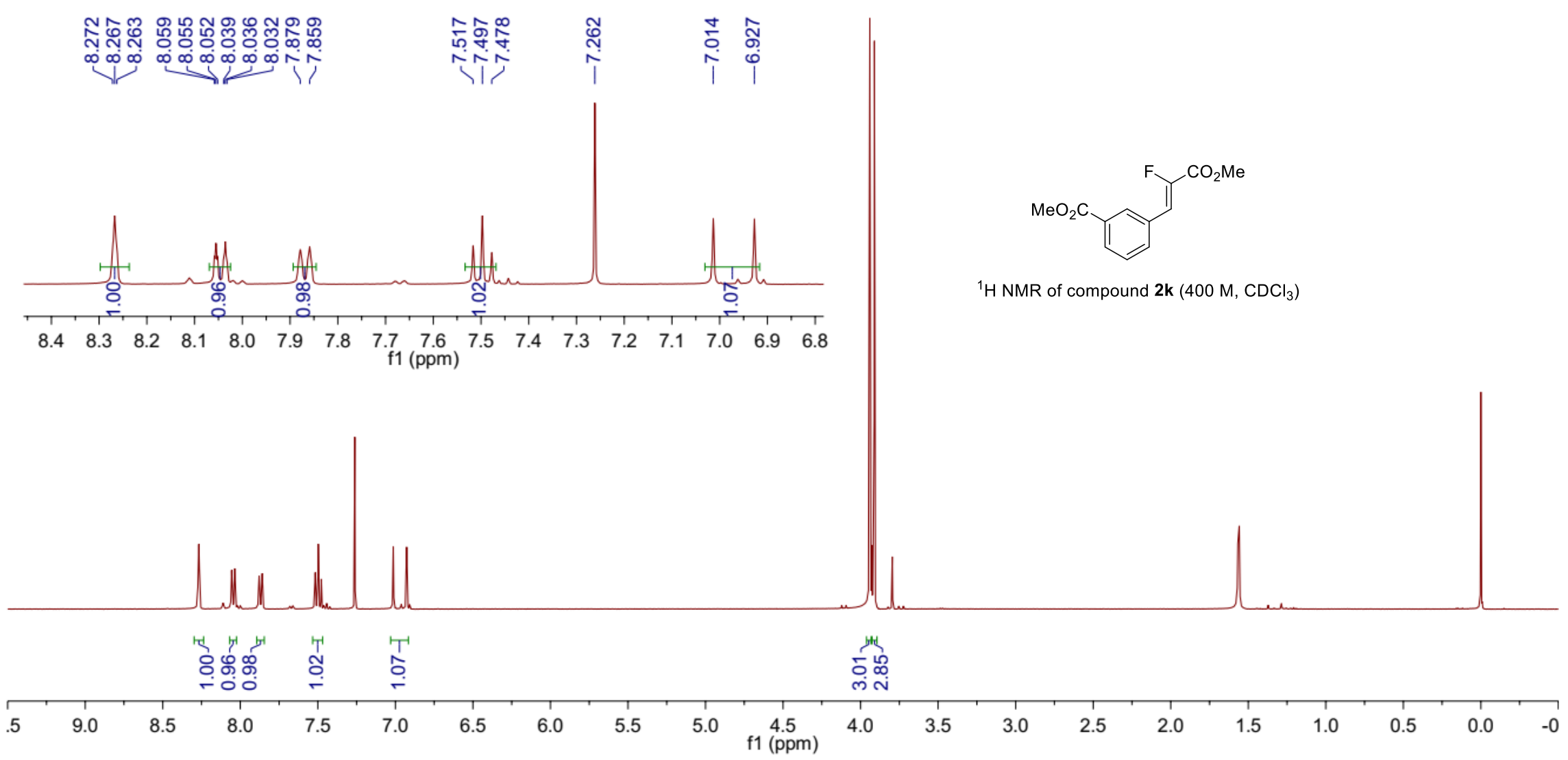


XSL-XI-84-400M. 11. fid

XSL-XI-84-400M

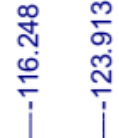

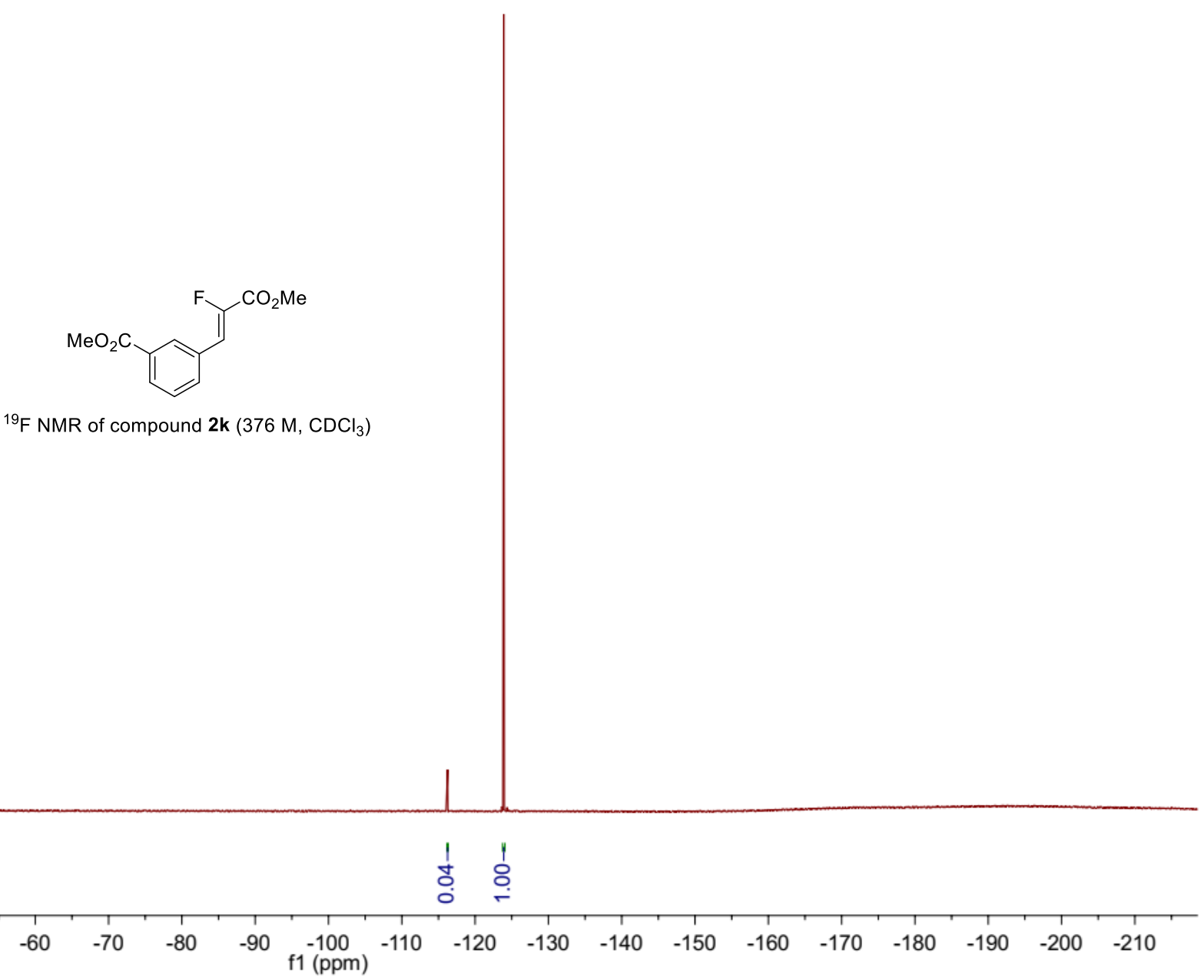




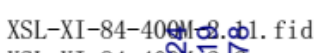
XSL-XI-84-40 औौ की के 닌

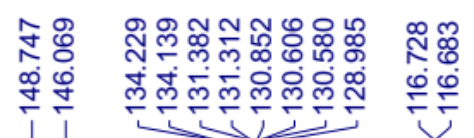
-

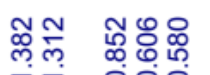$$
\text { m. }
$$

帝岕

m̄ं

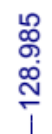

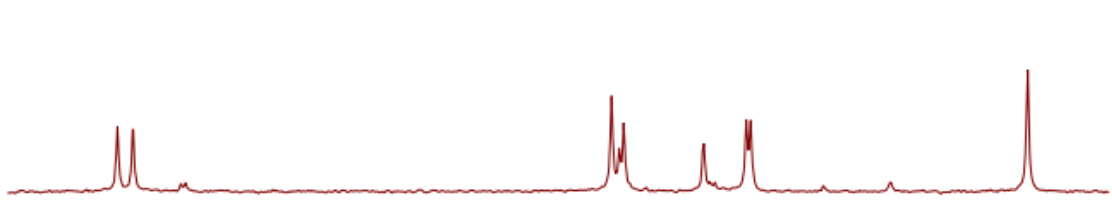

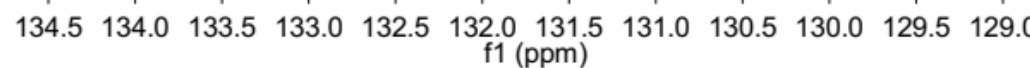
$\mathrm{f} 1$ (ppm)

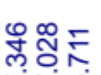

र्रंश
율류

กิ่

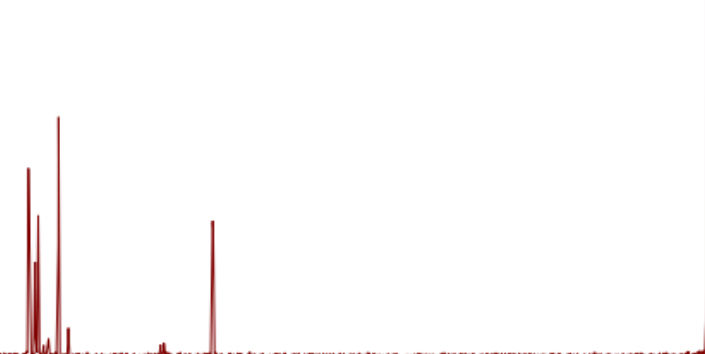

${ }^{13} \mathrm{C}$ NMR of compound $2 \mathbf{k}\left(100 \mathrm{M}, \mathrm{CDCl}_{3}\right)$
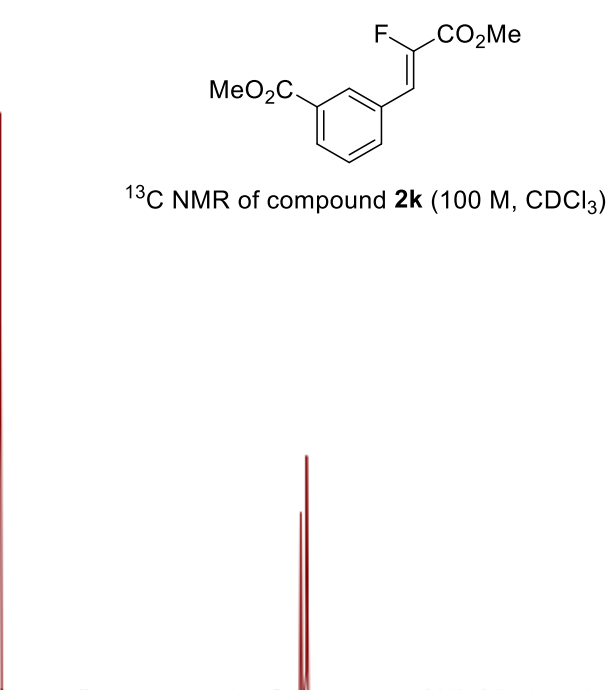


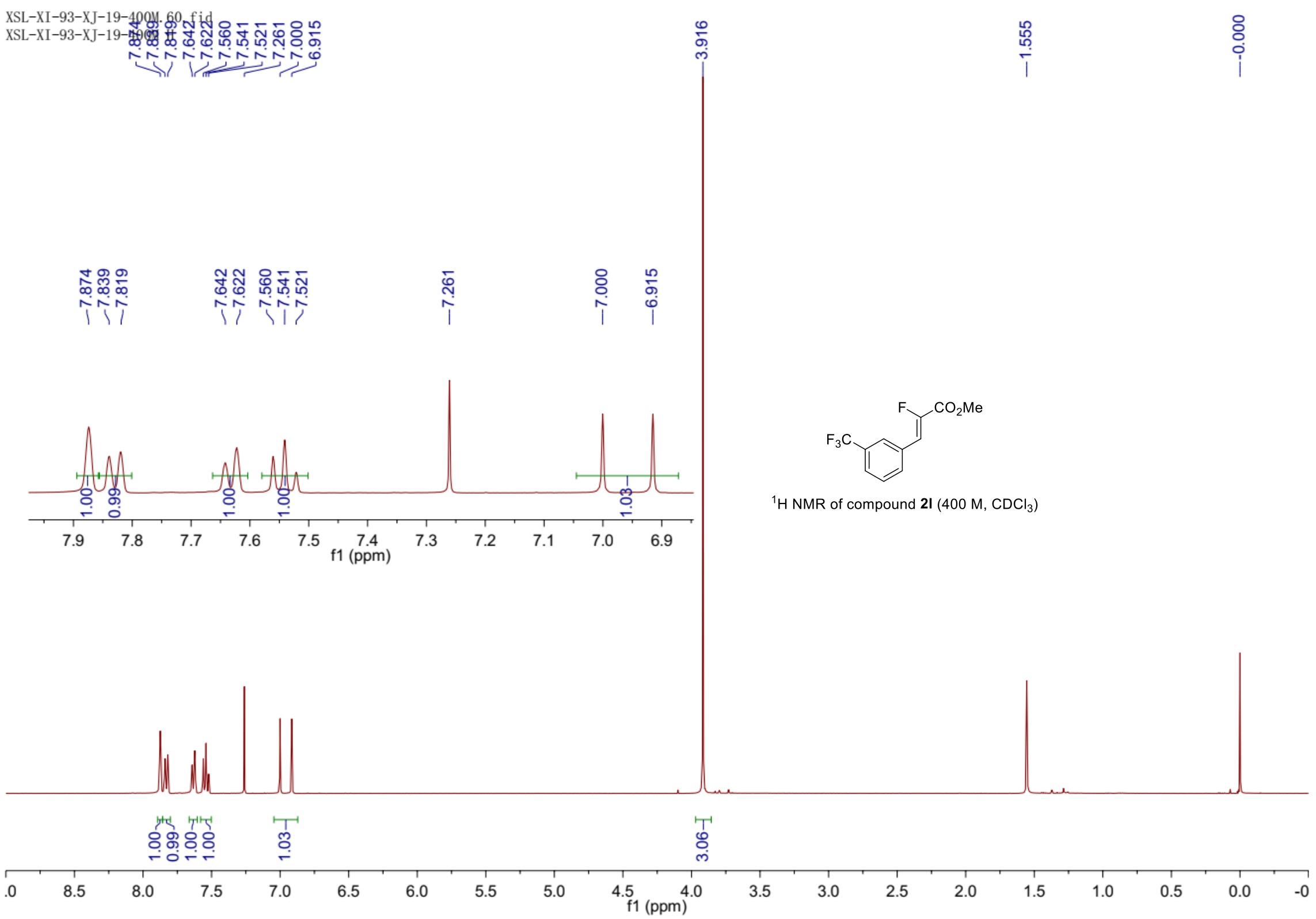


XSL-XI-93-XJ-19-400M. 62. fid XSL-XI-93-XJ-19-400M F

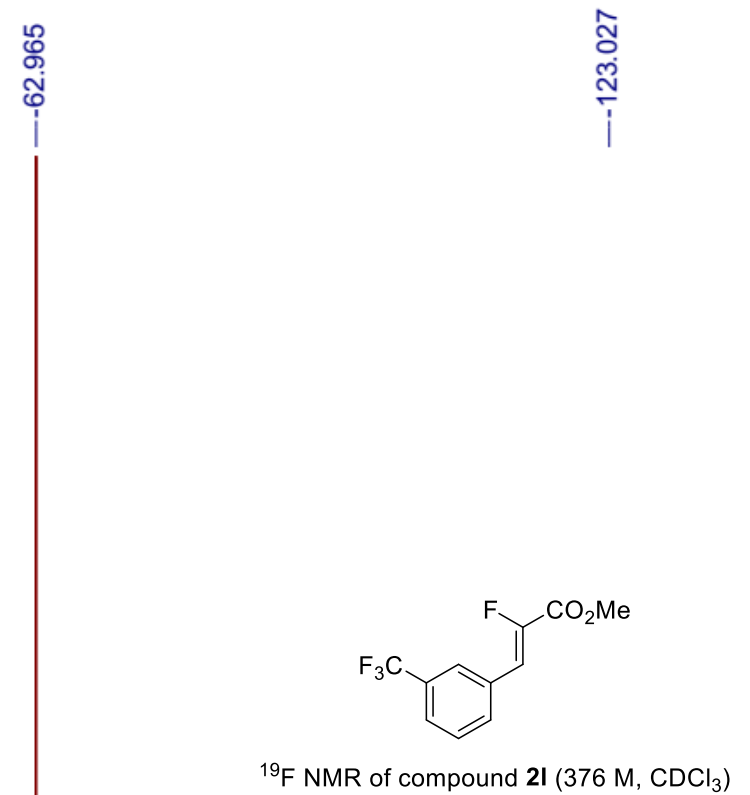

${ }^{19} \mathrm{~F} \mathrm{NMR}$ of compound $2 \mathrm{I}\left(376 \mathrm{M}, \mathrm{CDCl}_{3}\right)$

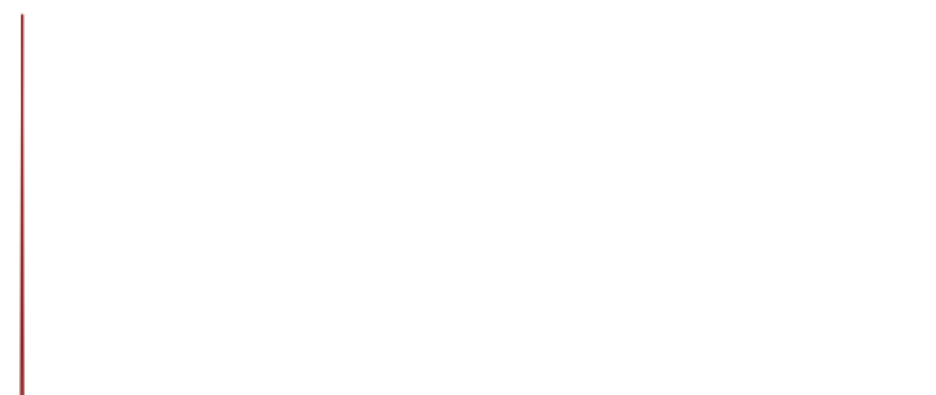




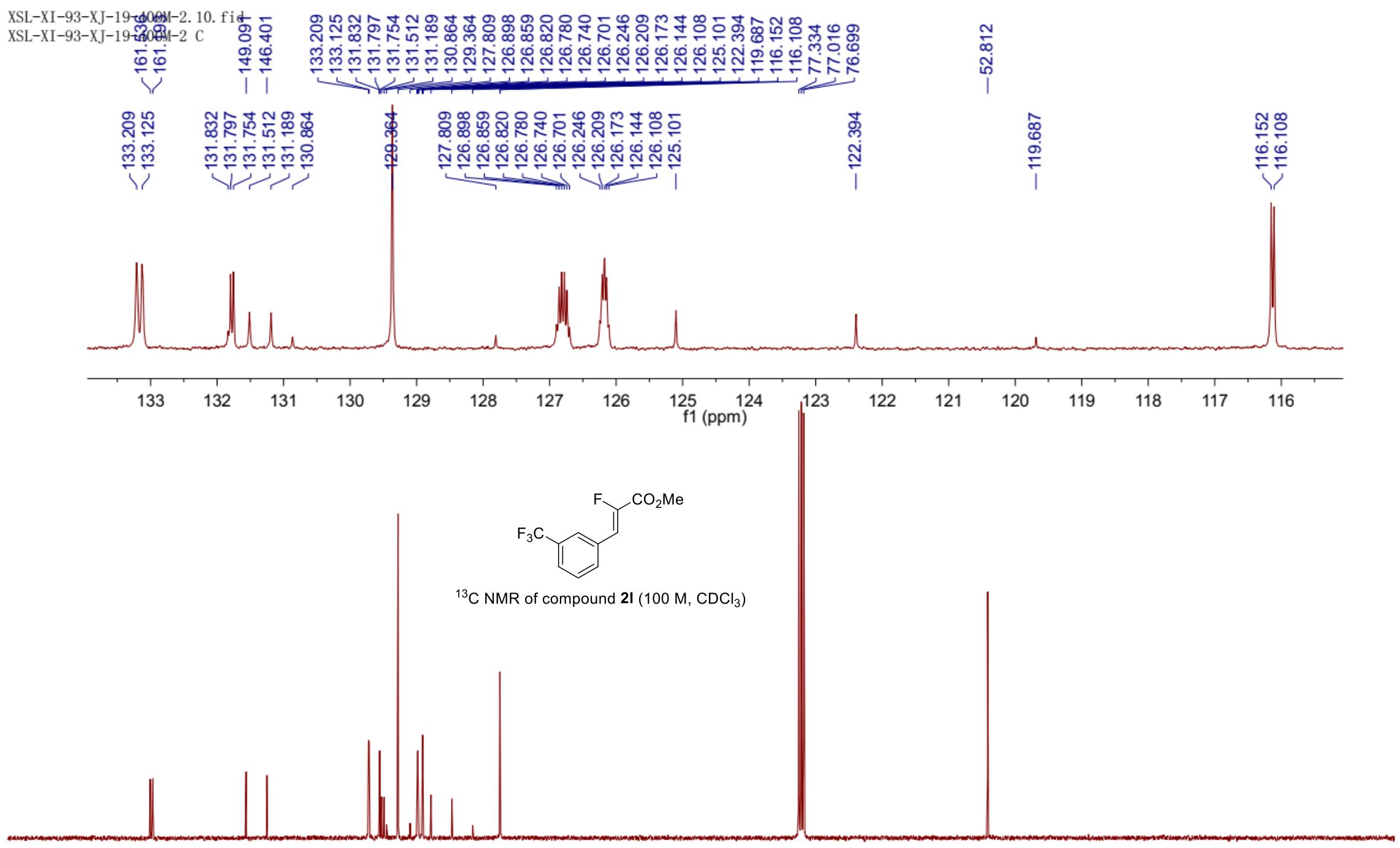

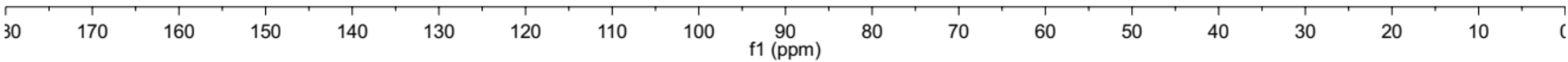




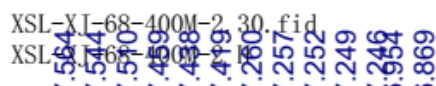

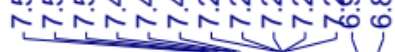
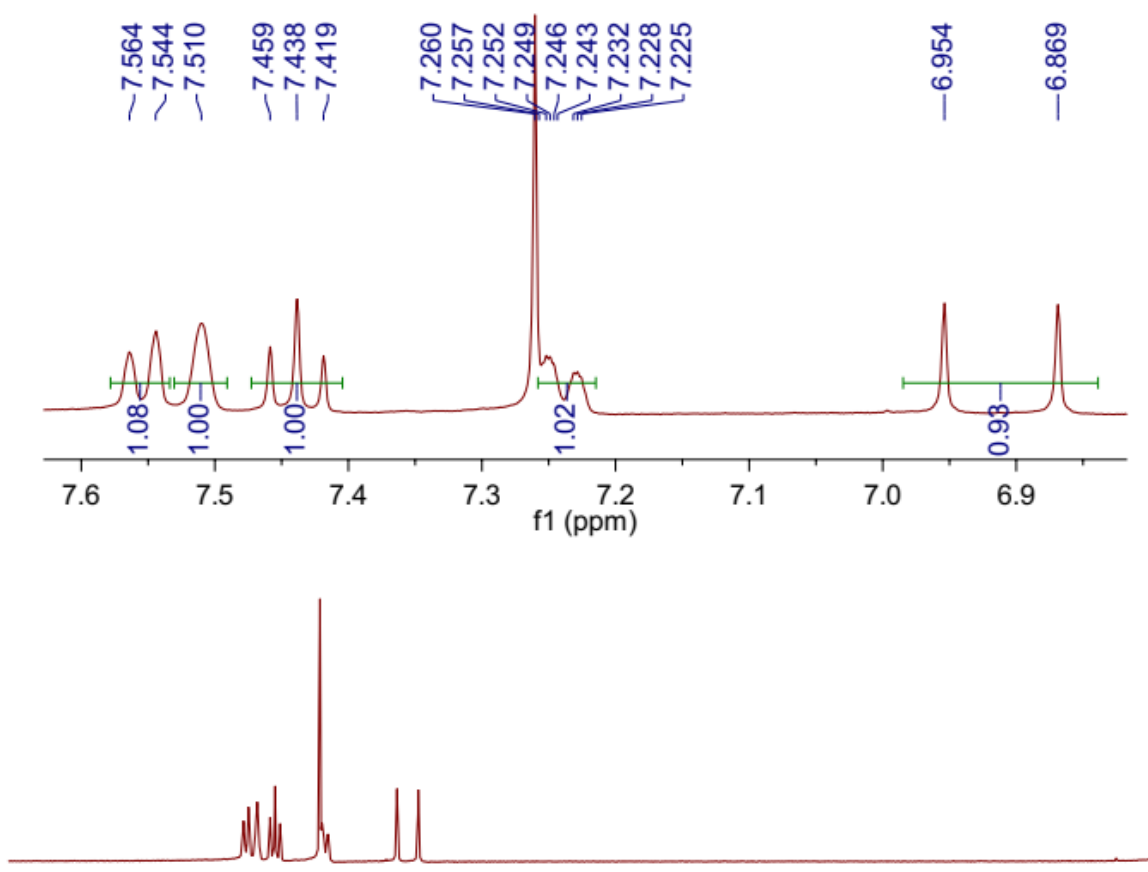

${ }^{1} \mathrm{H}$ NMR of compound $2 \mathrm{~m}\left(400 \mathrm{M}, \mathrm{CDCl}_{3}\right)$

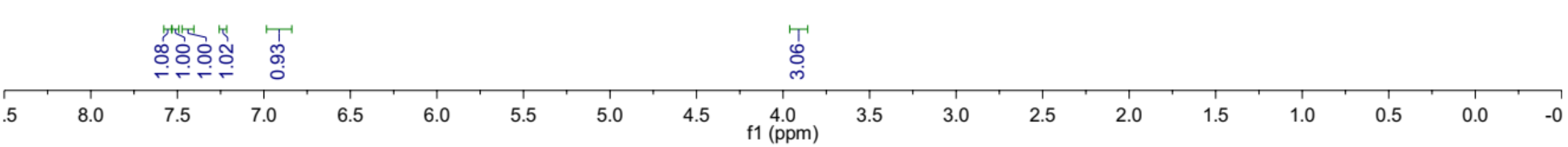


XSL-XJ-68-400M-2. 32. fid

XSL-XJ-68-400M-2 F

i

${ }^{19} \mathrm{~F} \mathrm{NMR}$ of compound $2 \mathrm{~m}\left(376 \mathrm{M}, \mathrm{CDCl}_{3}\right)$

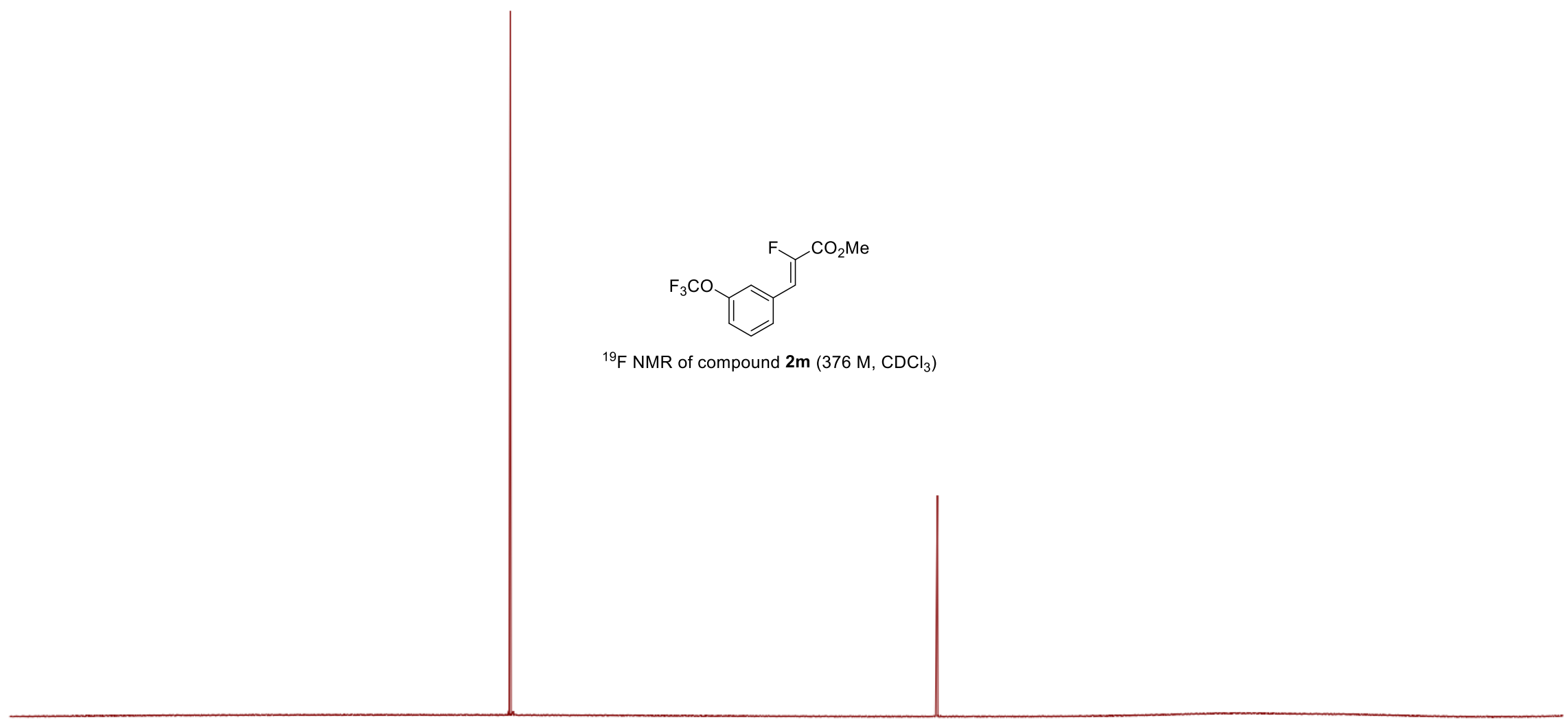

$\begin{array}{lllllllllllllllllllllll}10 & 0 & -10 & -20 & -30 & -40 & -50 & -60 & -70 & -80 & -90 & -100 & -110 & -120 & -130 & -140 & -150 & -160 & -170 & -180 & -190 & -200 & -210\end{array}$ 


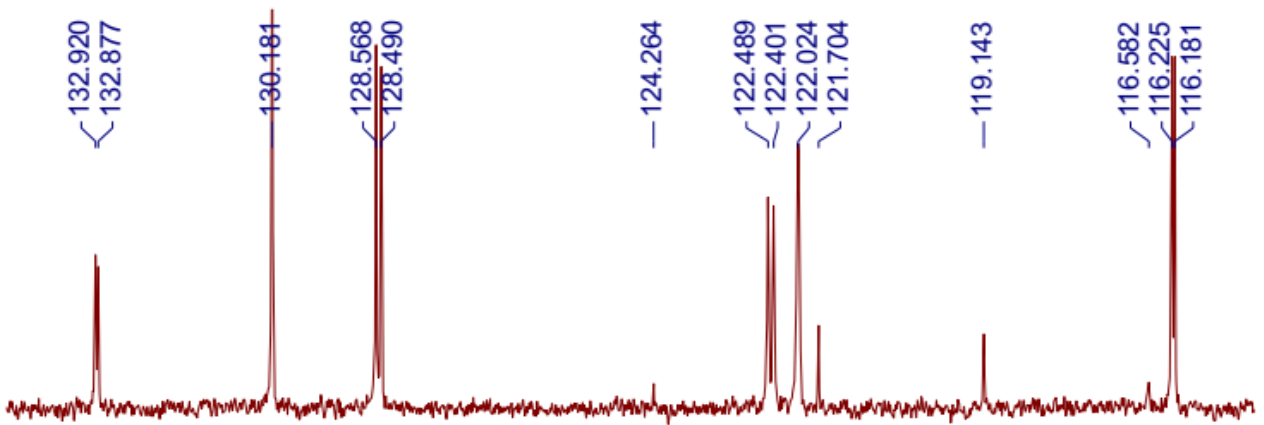

$\begin{array}{llllllllllllllllllll}134 & 133 & 132 & 131 & 130 & 129 & 128 & 127 & 126 & 125 & 124 & 123 & 122 & 121 & 120 & 119 & 118 & 117 & 116 & 11\end{array}$ $\mathrm{f} 1(\mathrm{ppm})$

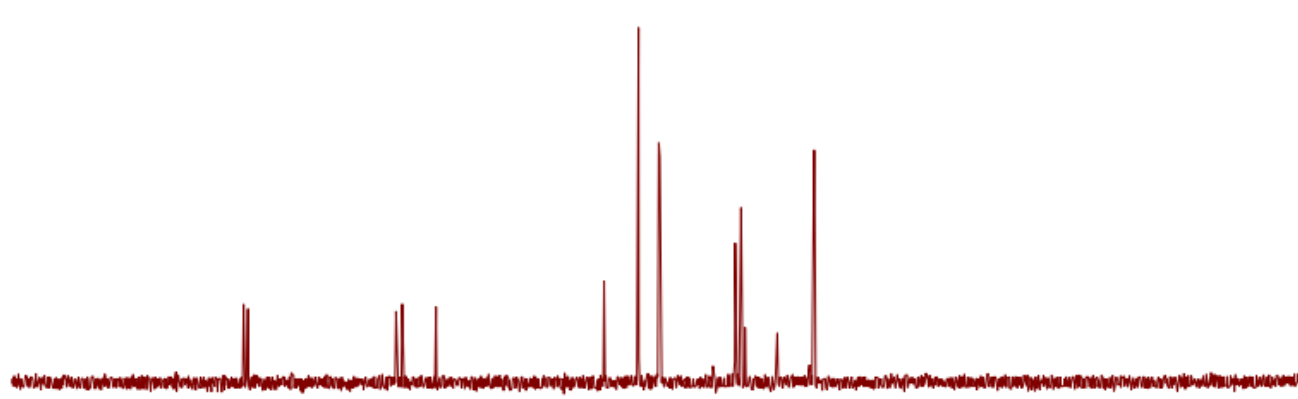

${ }^{13} \mathrm{C}$ NMR of compound $2 \mathrm{~m}\left(100 \mathrm{M}, \mathrm{CDCl}_{3}\right)$

$30 \quad 170 \quad 160 \quad 150$

130

120

110

100

$\stackrel{90}{\mathrm{f} 1}(\mathrm{ppm})$

80

70

60

50

40

30 


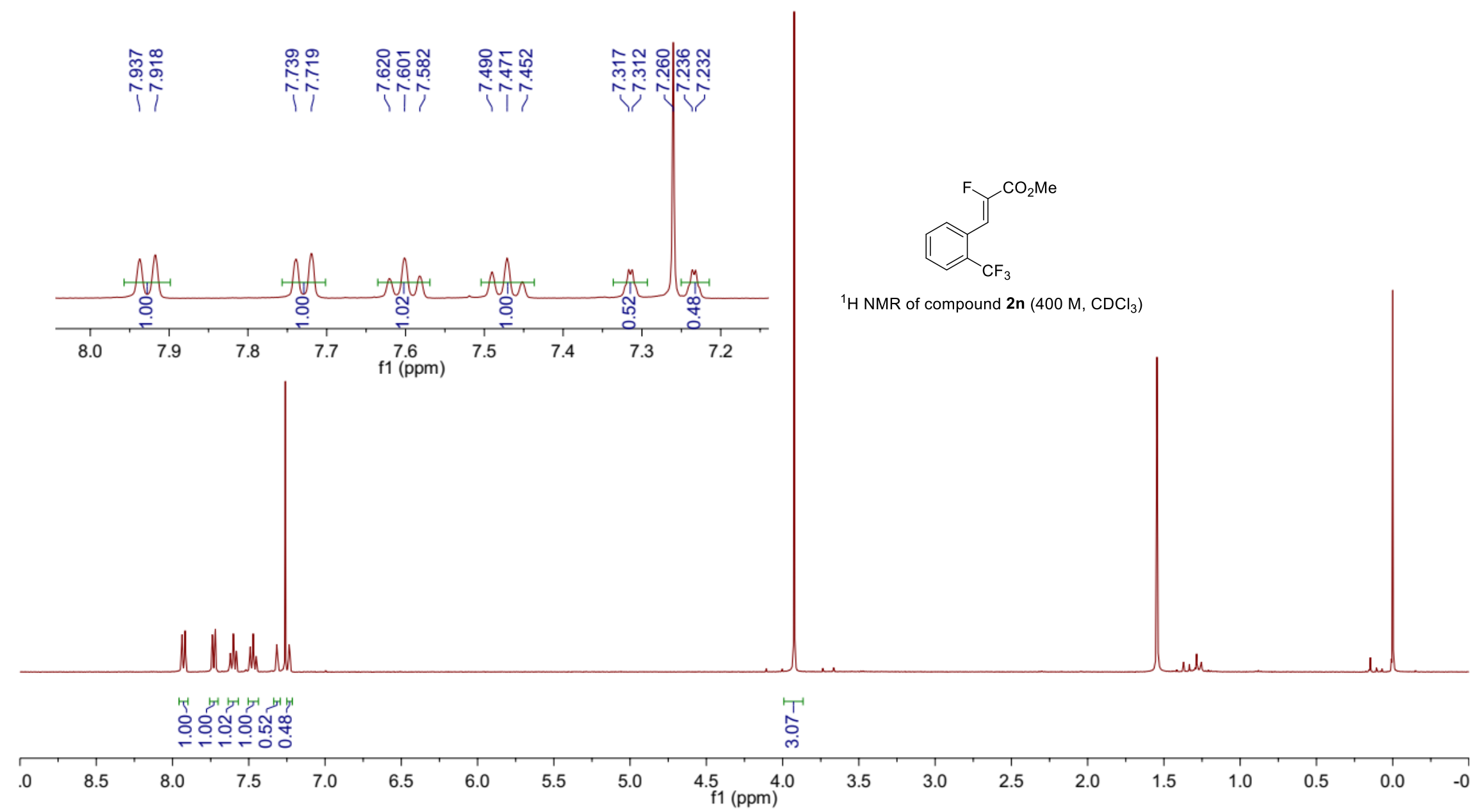


XSL-XI-112-XJ-17-400M. 42. fid

XSL-XI-112-XJ-17-400M F

啳兽

คุ

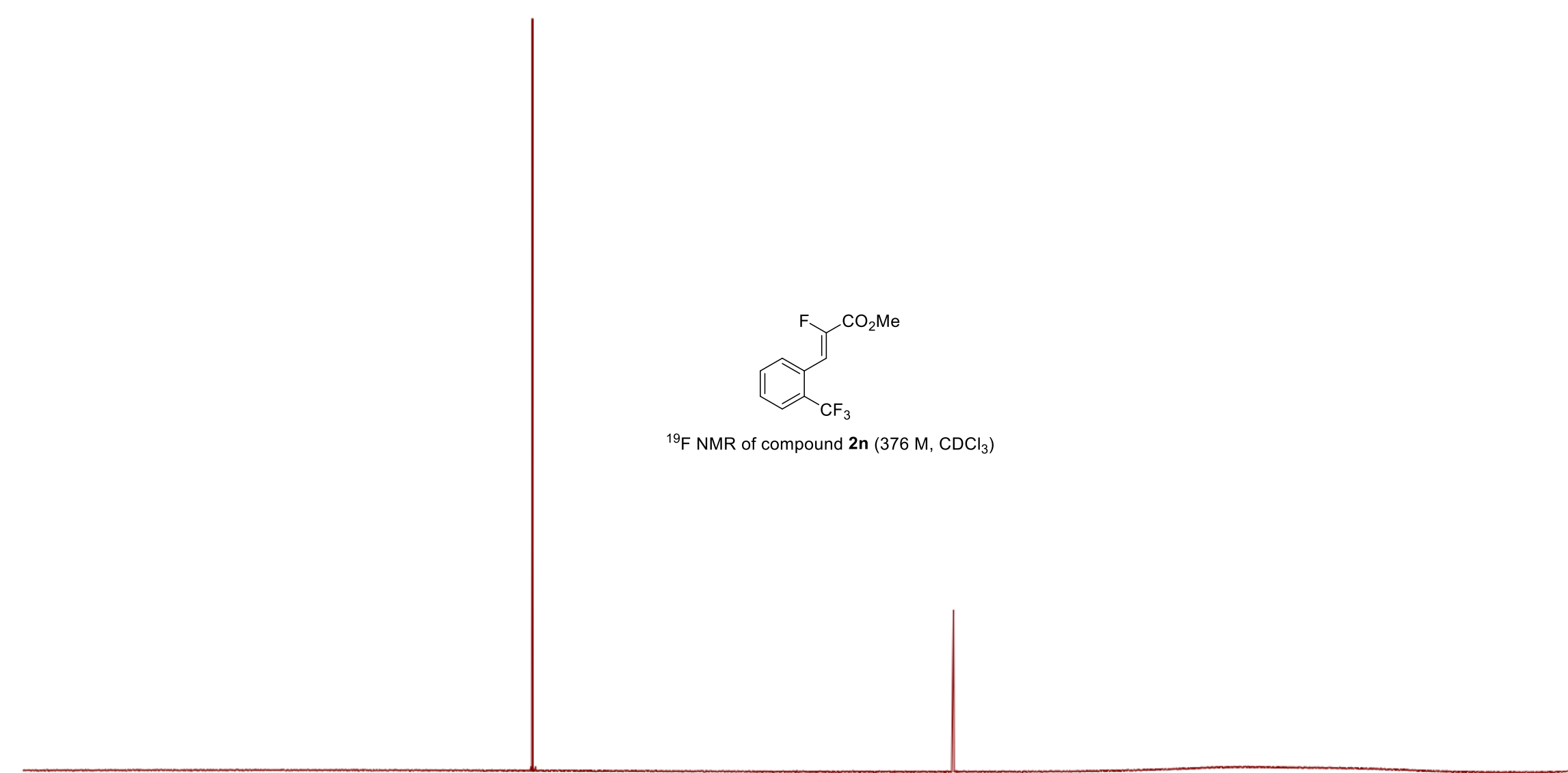

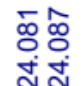

곤

广 


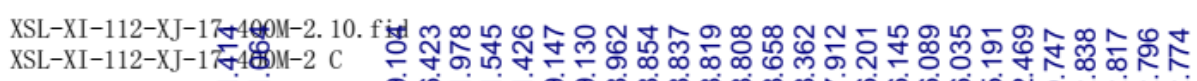

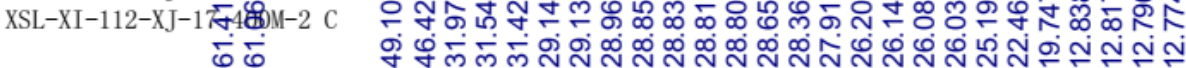

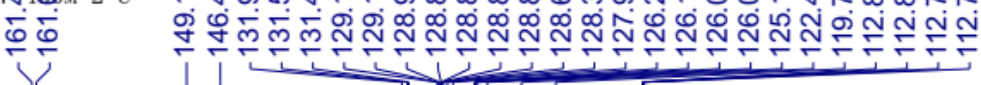
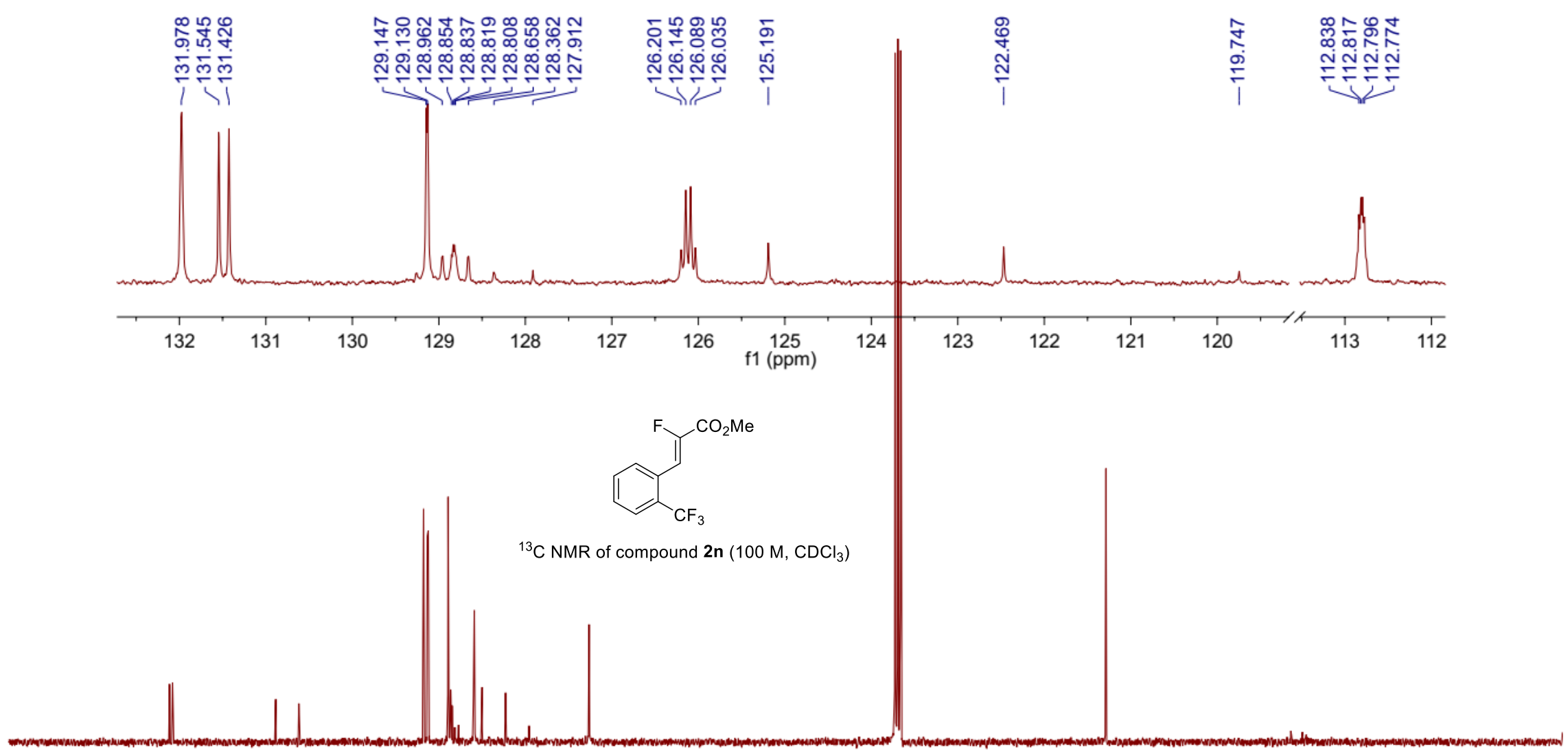

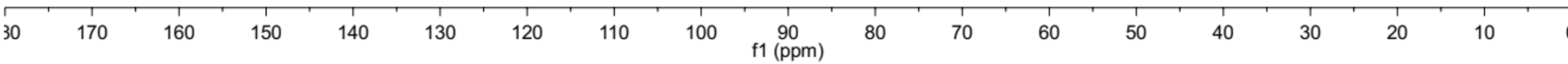




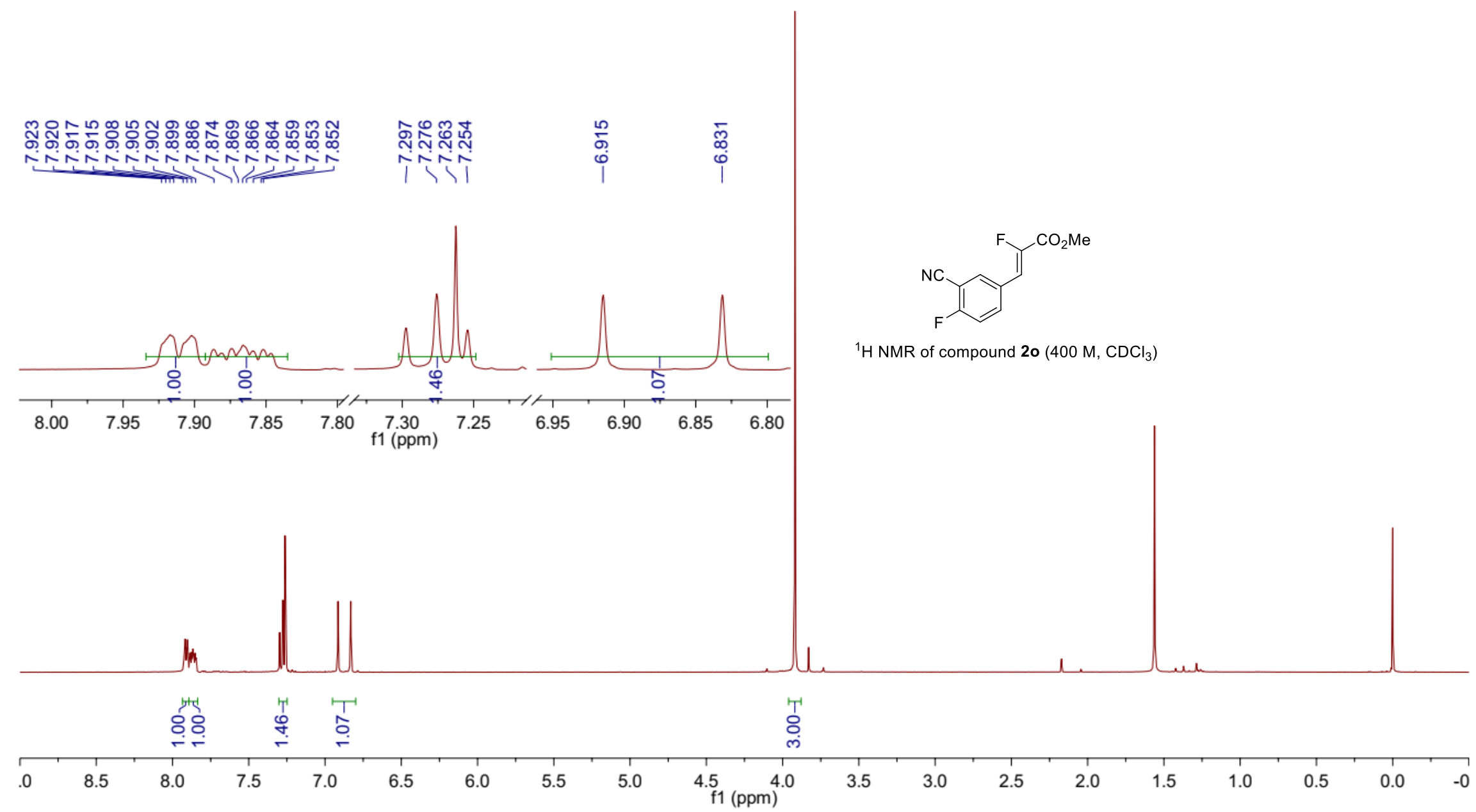


XSL-XI-96-400M. 11. fid

XSL-XI-96-400M $F$

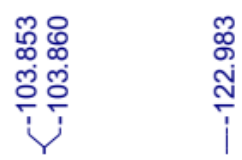

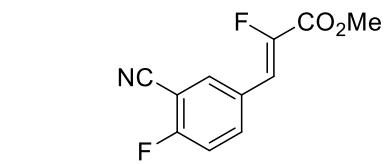

${ }^{19} \mathrm{~F} \mathrm{NMR}$ of compound $20\left(376 \mathrm{M}, \mathrm{CDCl}_{3}\right)$

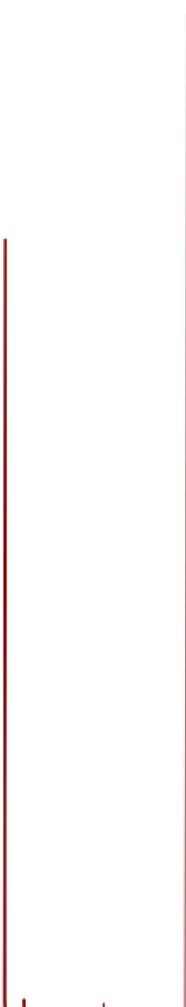




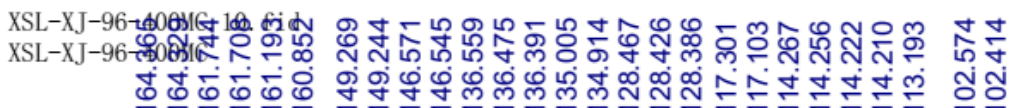

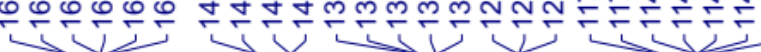

.

员㖞
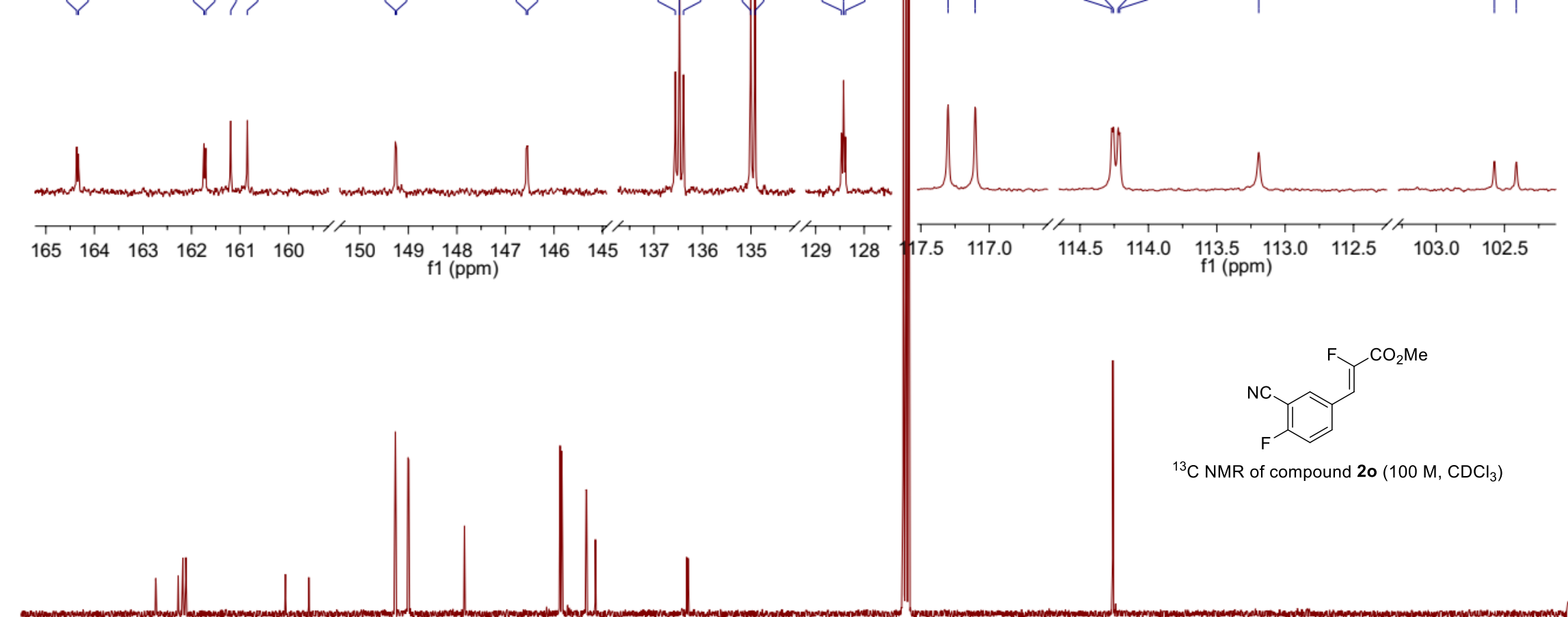

${ }^{13} \mathrm{C}$ NMR of compound $20\left(100 \mathrm{M}, \mathrm{CDCl}_{3}\right)$ 


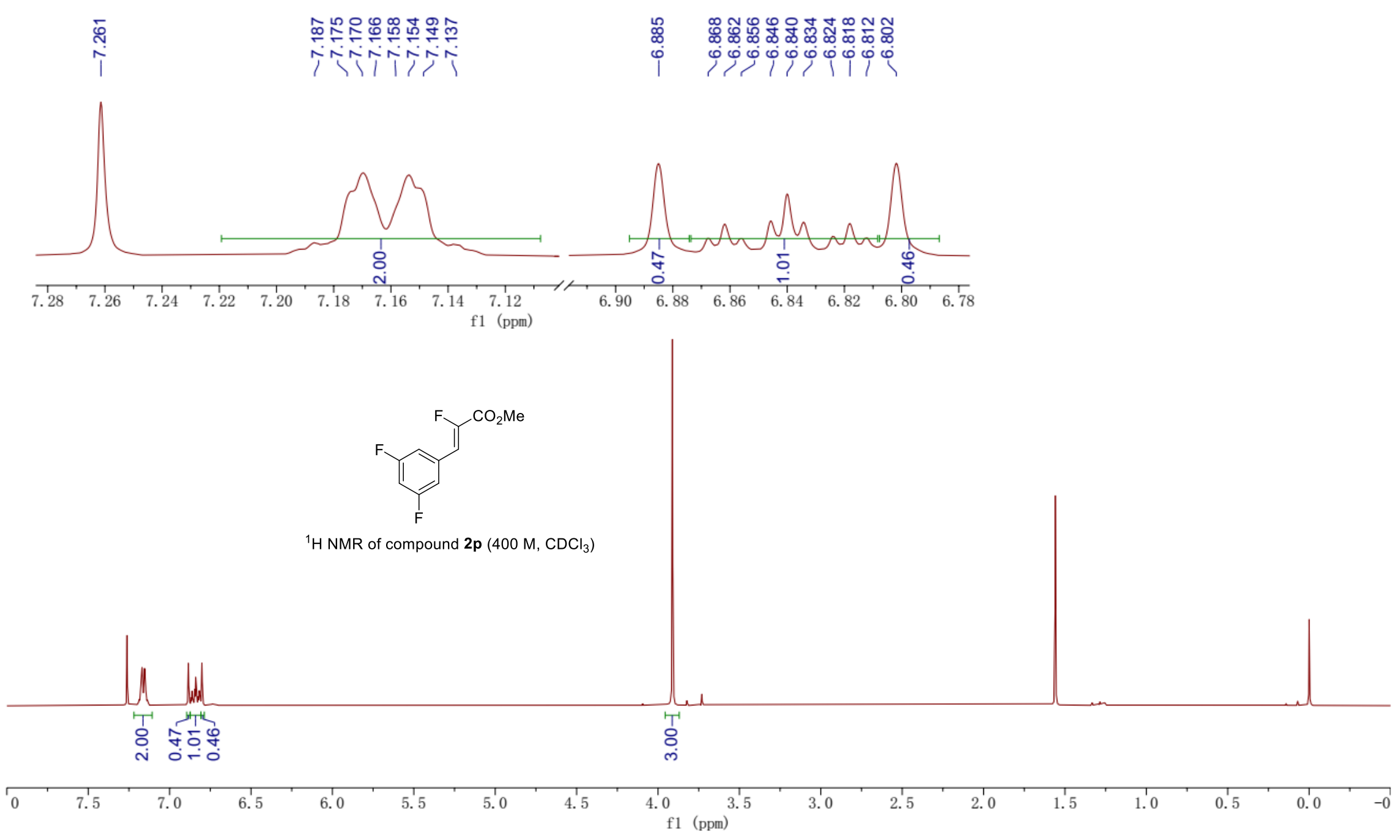


XSL-XJ-97-400M. 11. fid

XSL-XJ-97-400M

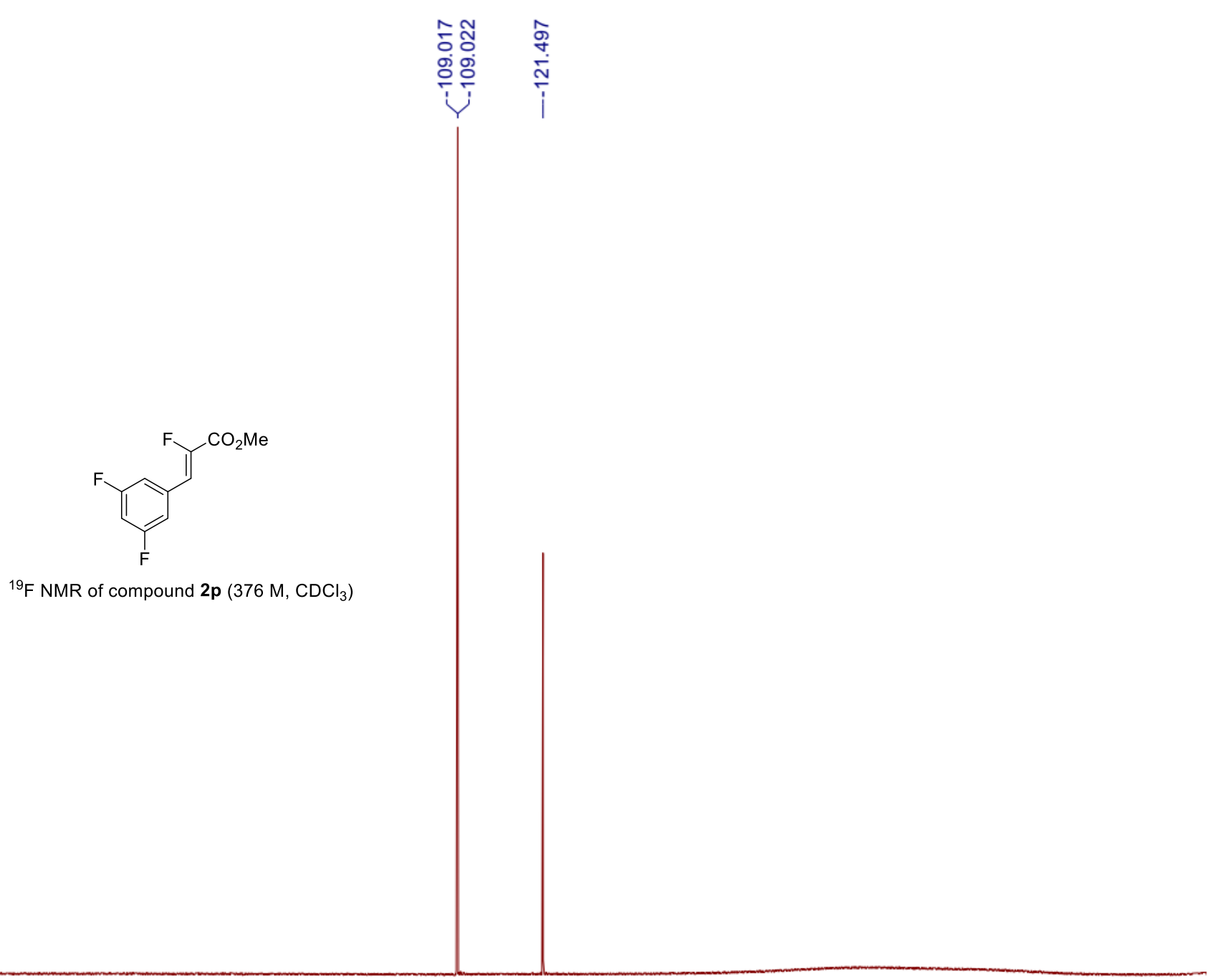




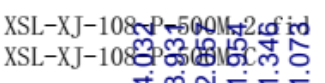

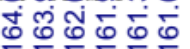

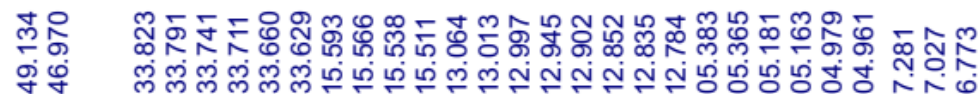

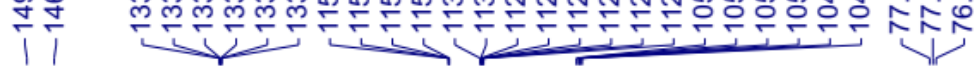

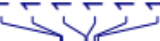

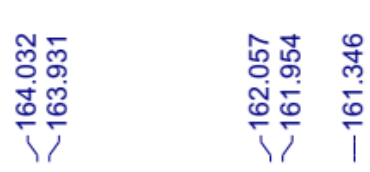

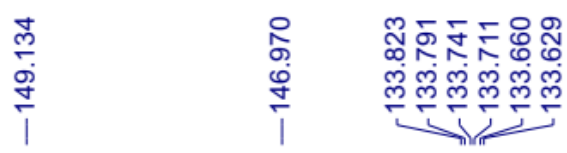

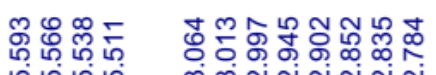

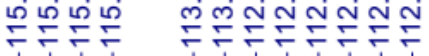

ஹ్ֻ

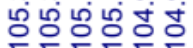
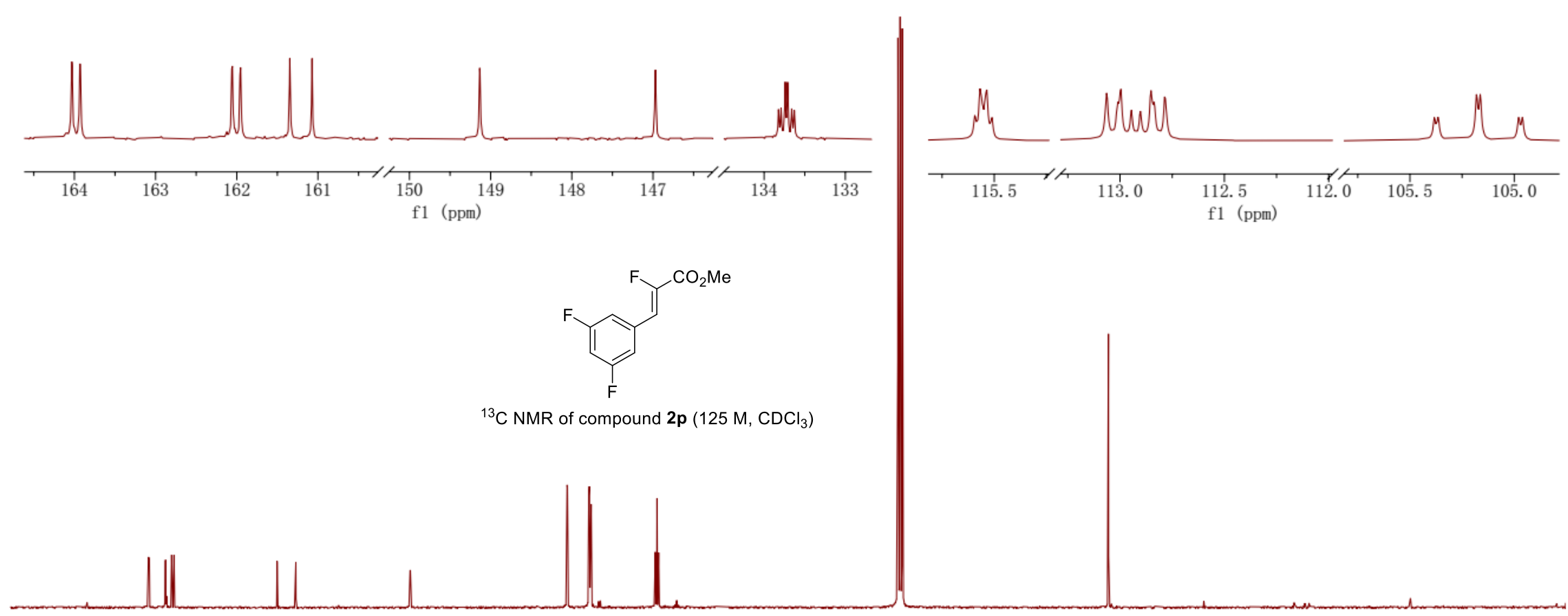

30

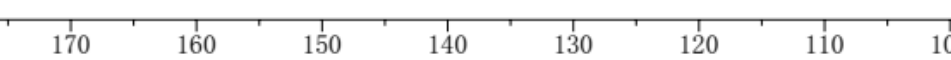




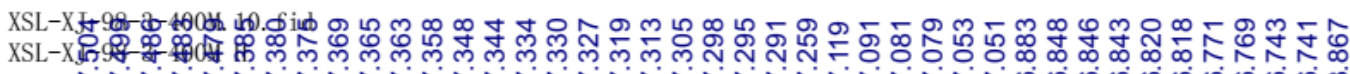

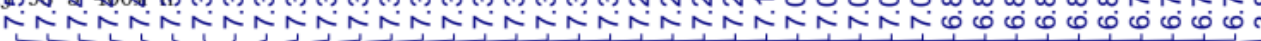

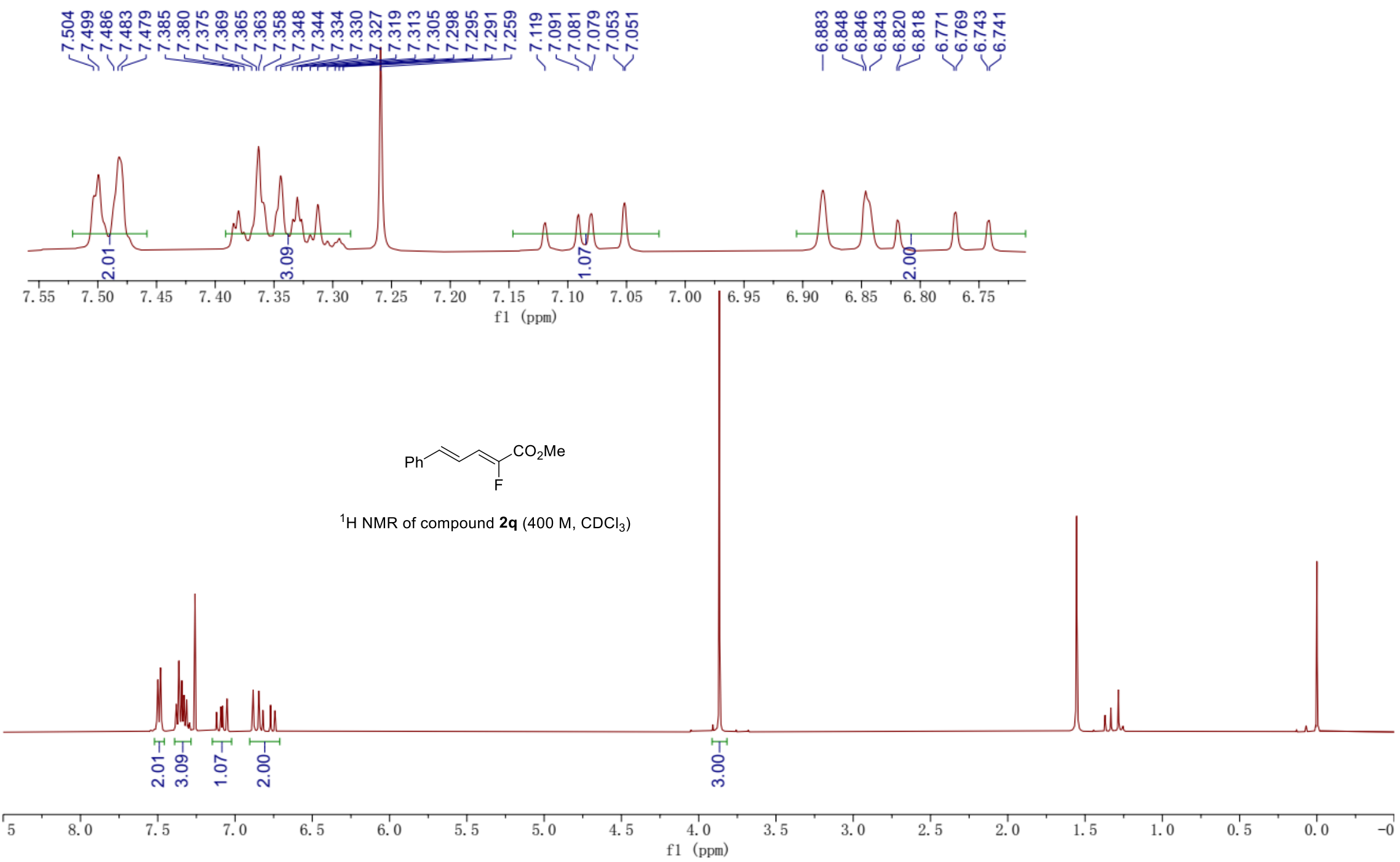


XSL-XJ-98-4-400M. 11. fid

XSL-XJ-98-4-400M

莒

$\stackrel{\infty}{\stackrel{\infty}{\sim}}$

$\mathrm{Ph}_{\mathrm{F}}^{\mathrm{CO}_{2} \mathrm{Me}}$

${ }^{19} \mathrm{~F}$ NMR of compound $2 \mathbf{q}\left(376 \mathrm{M}, \mathrm{CDCl}_{3}\right)$

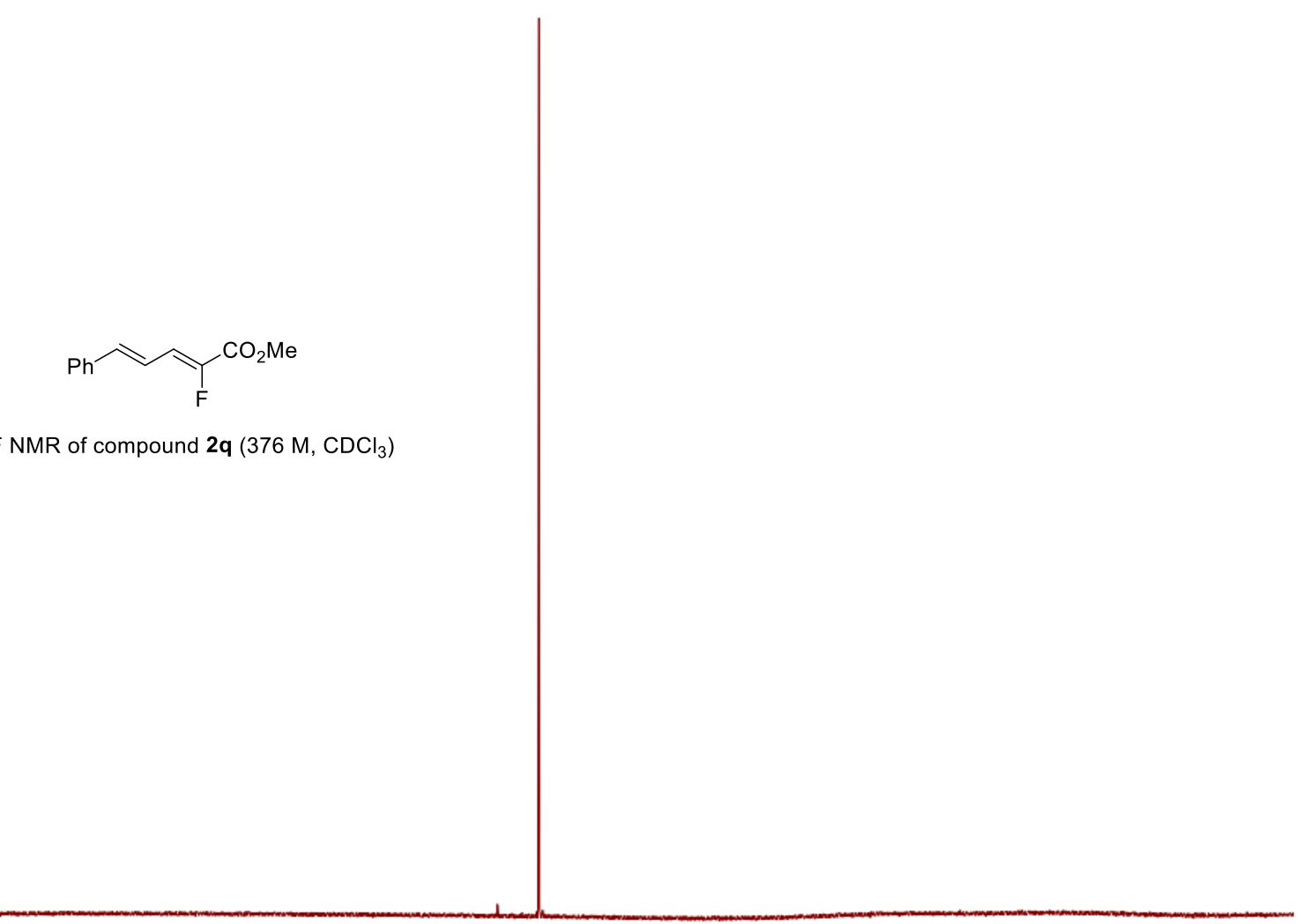

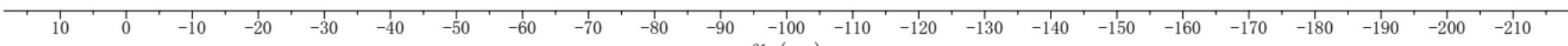
f1 (ppm) 
XSL-XJ-98-400MC.ภำ fid

एँ

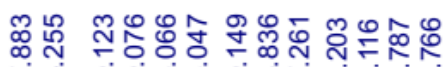

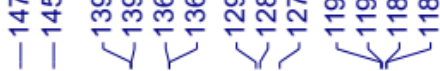
XSL-XJ-98-400MC ळै ले

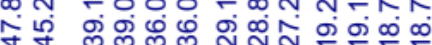

율ㅁำ

र्र人ष

学

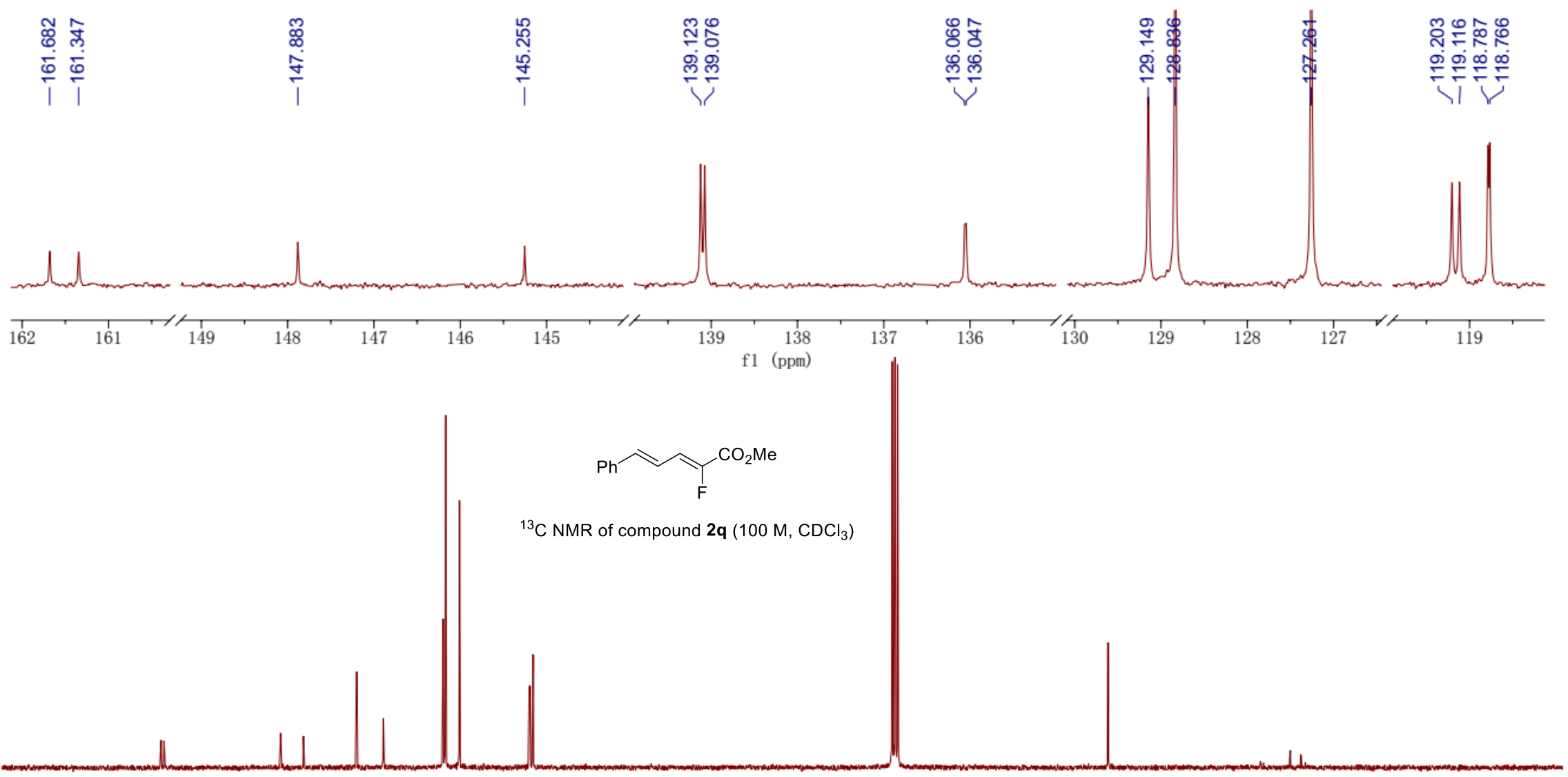

$30 \quad 170$
$150 \times 140$
110 


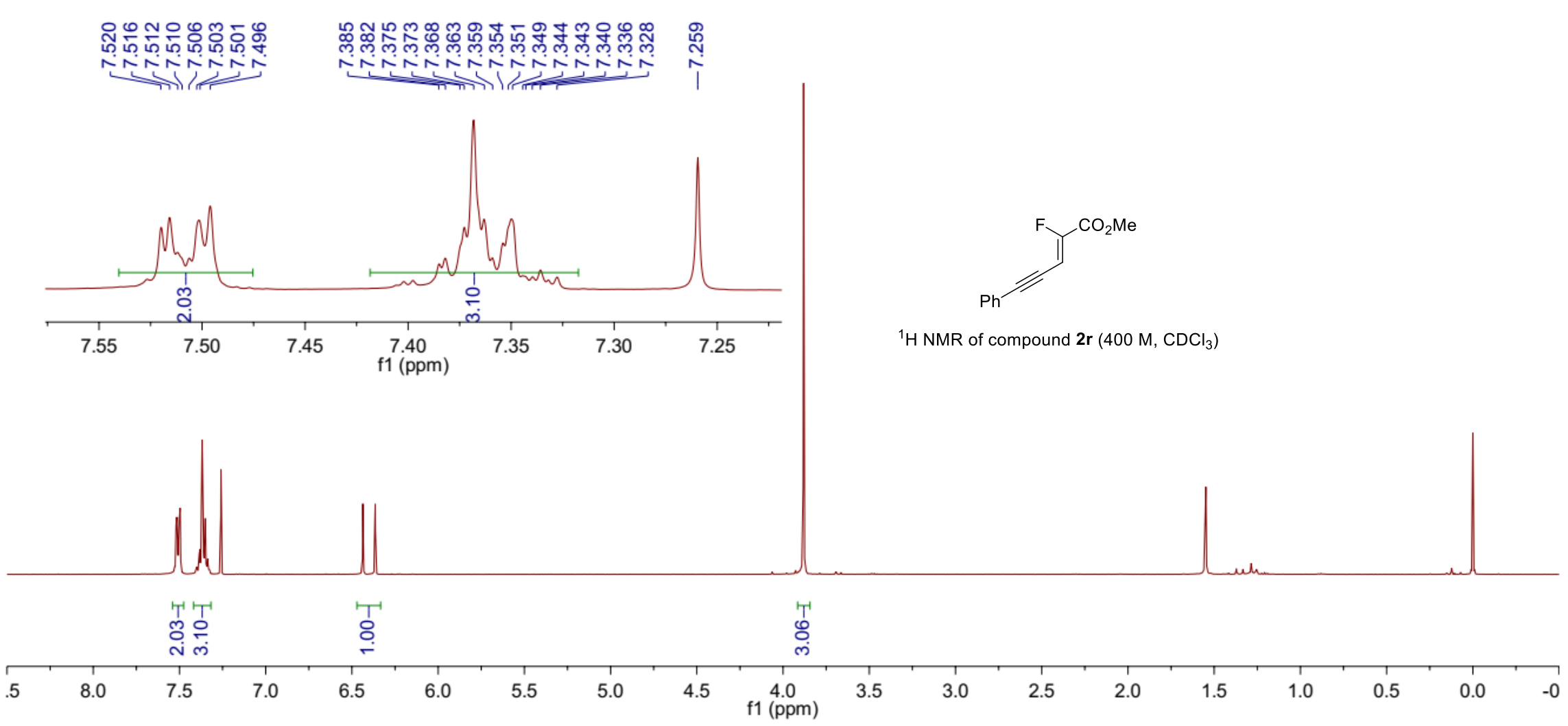


XSL-XJ-25-400M. 92. fid

XSL-XJ-25-400M F

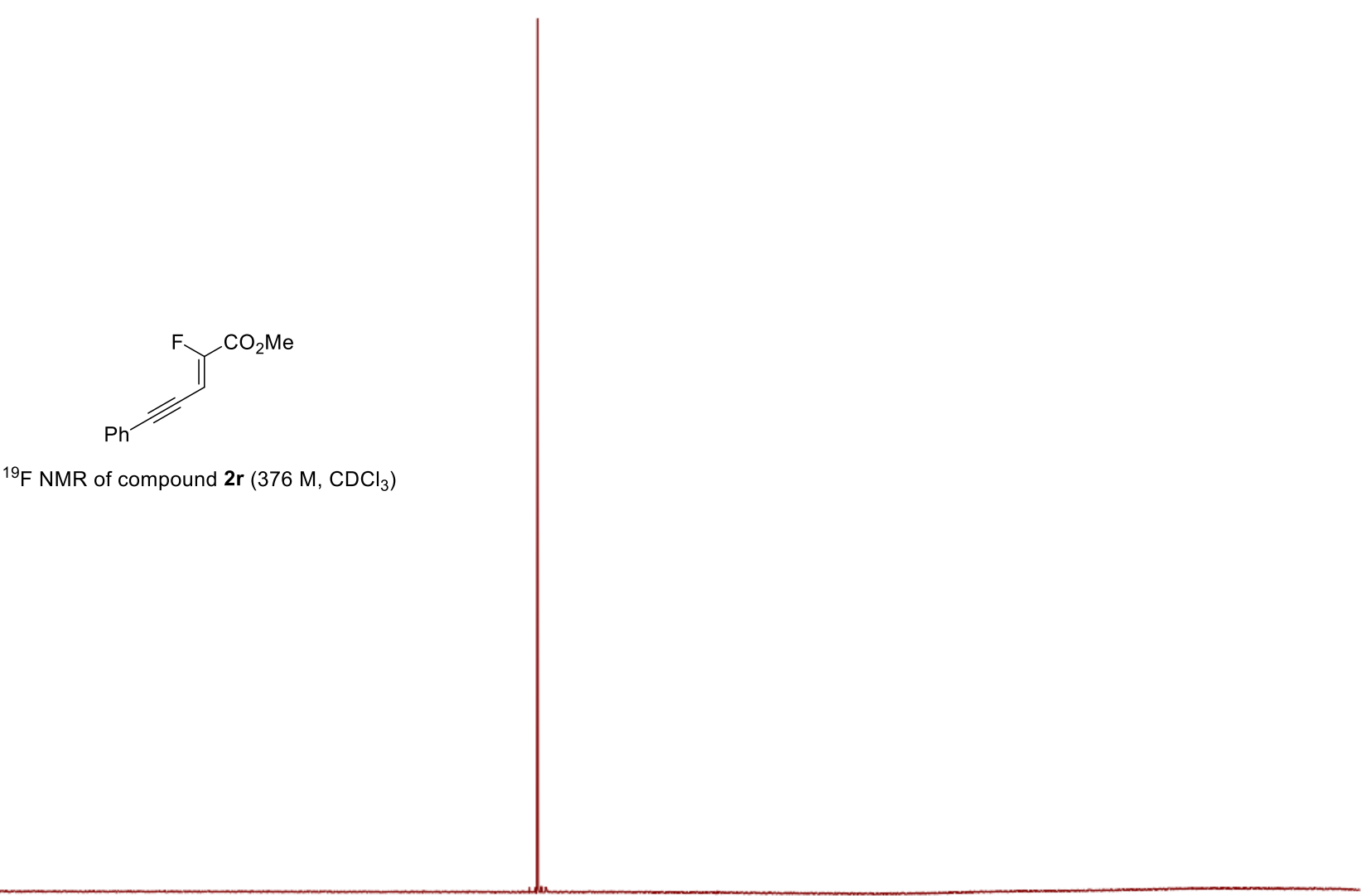

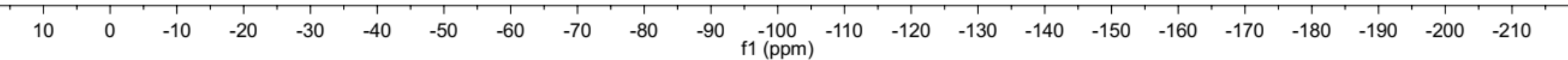




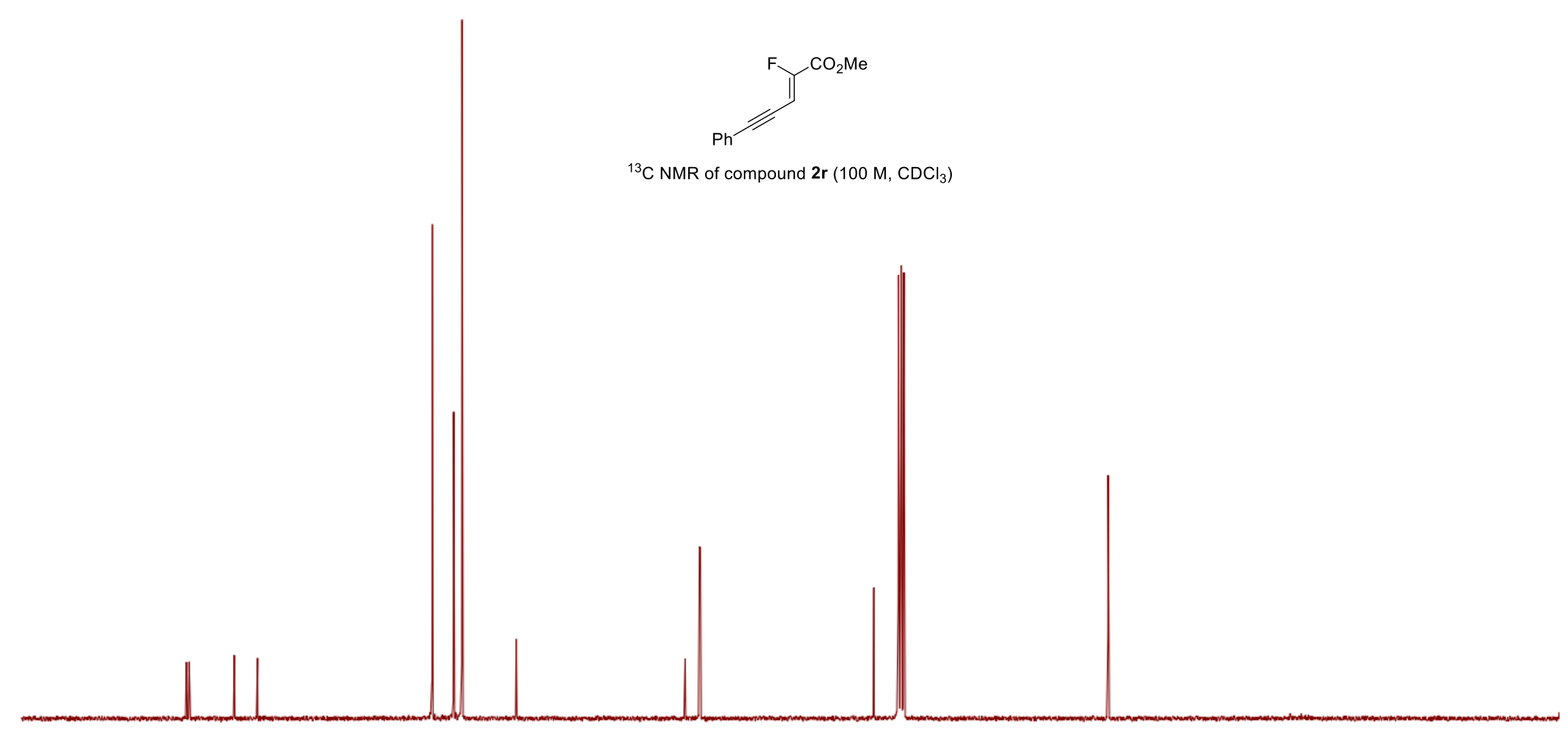

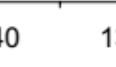
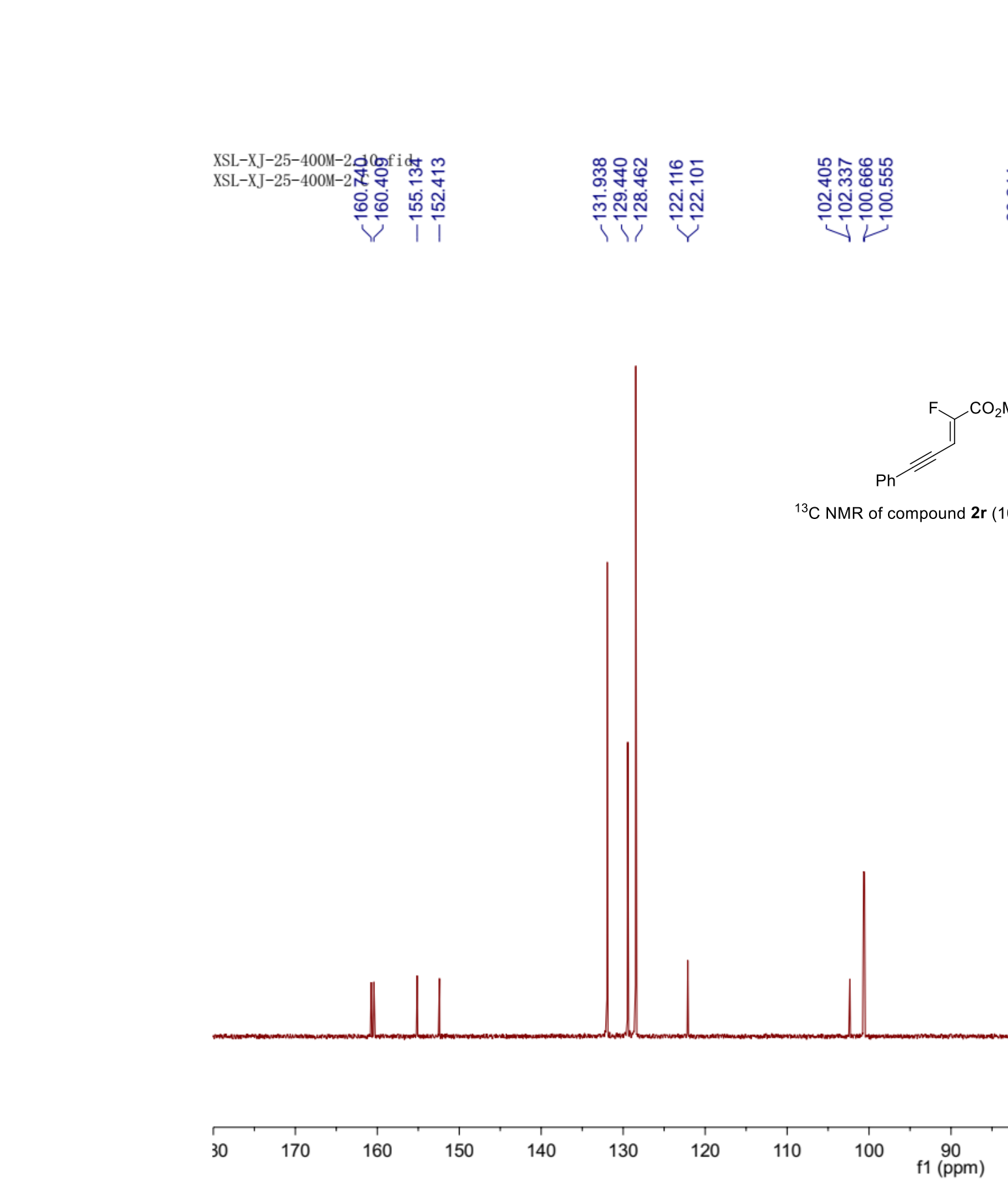
$(2$ 


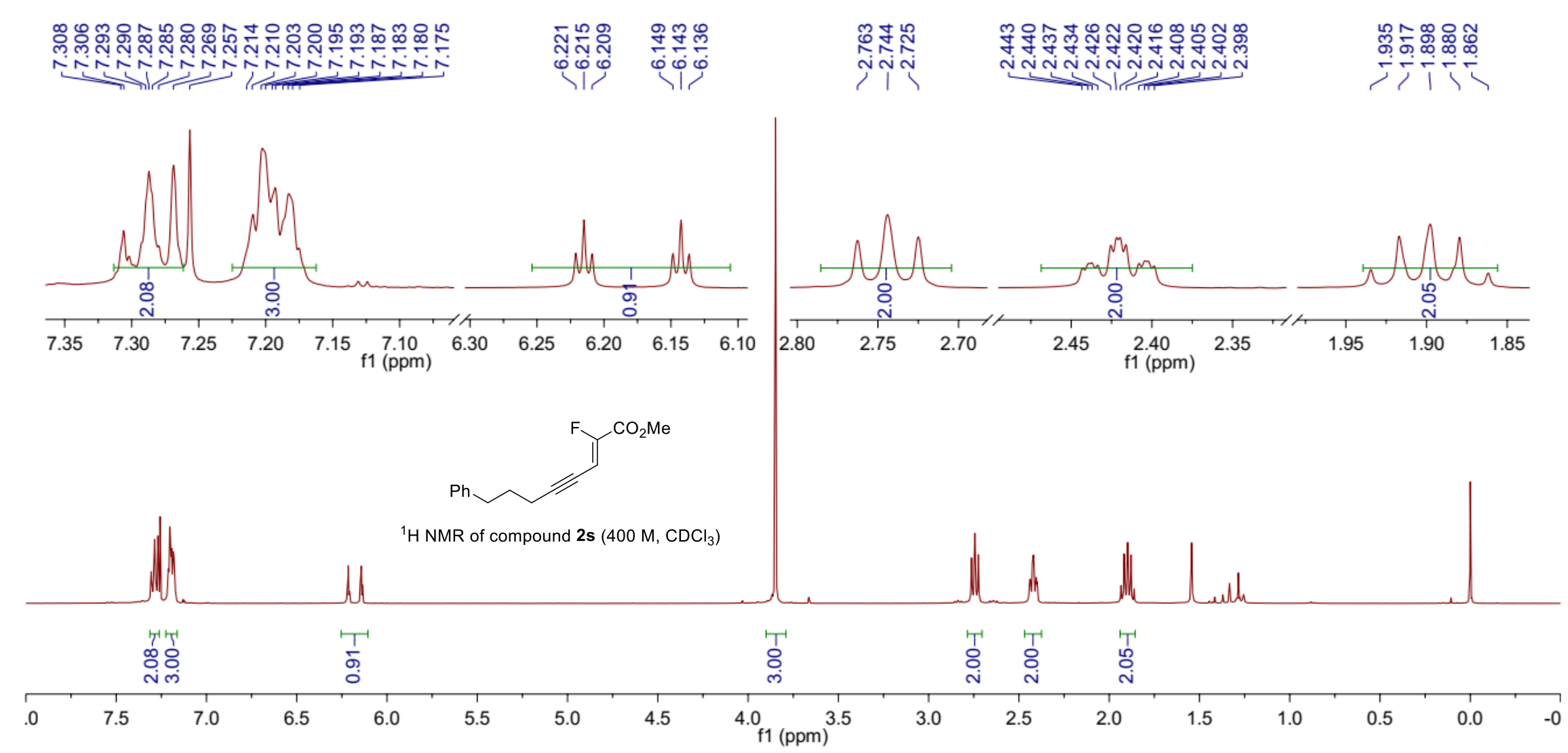


XSL-XJ-60-400M-2. 11. fid

XSL-XJ-60-400M-2 F

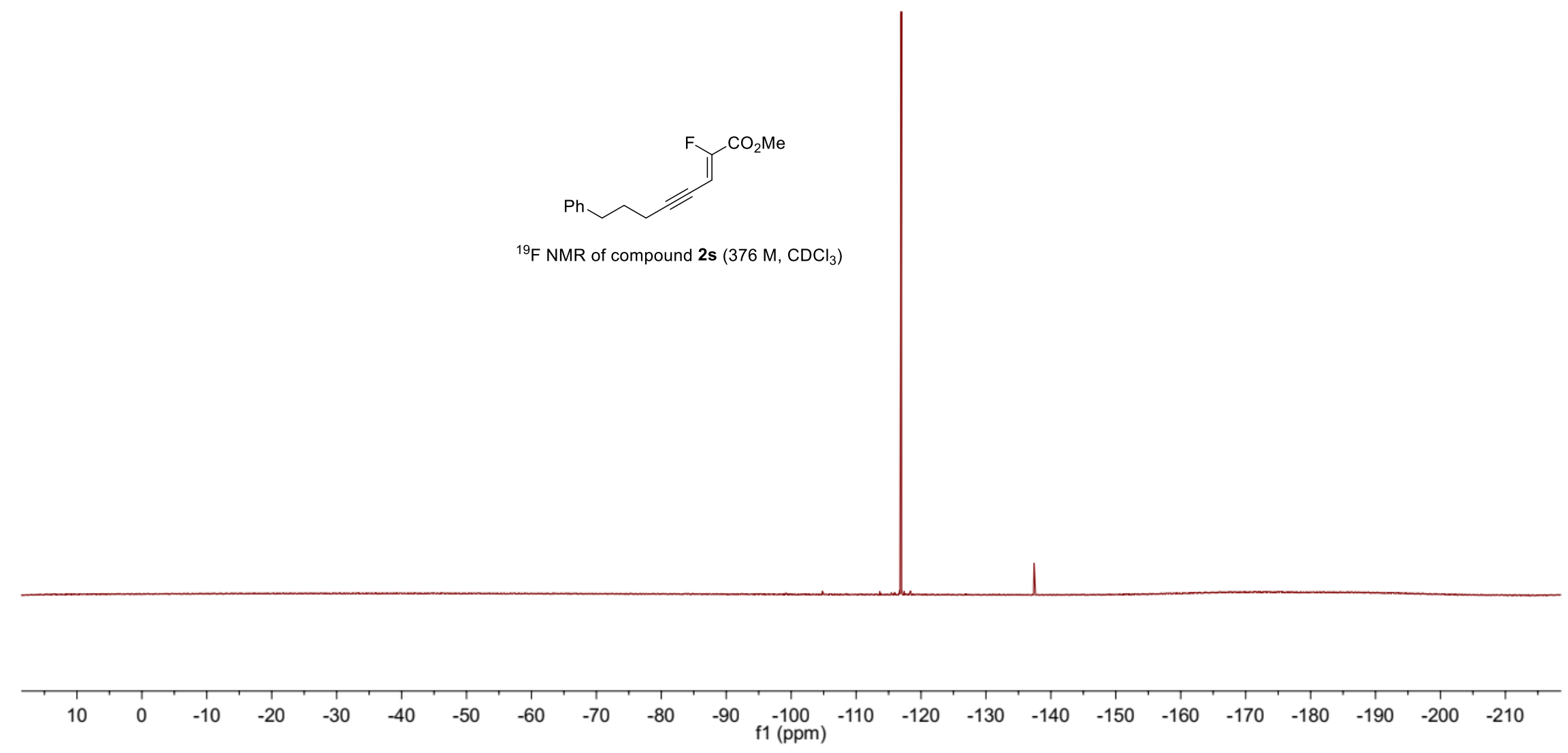




\begin{tabular}{|c|c|c|c|c|c|c|}
\hline 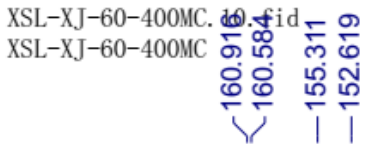 & 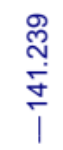 & 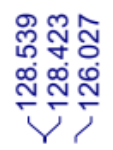 & 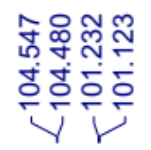 & 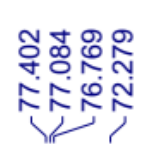 & $\begin{array}{l}2 \\
0 \\
0 \\
i n\end{array}$ & 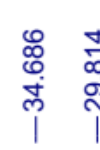 \\
\hline
\end{tabular}

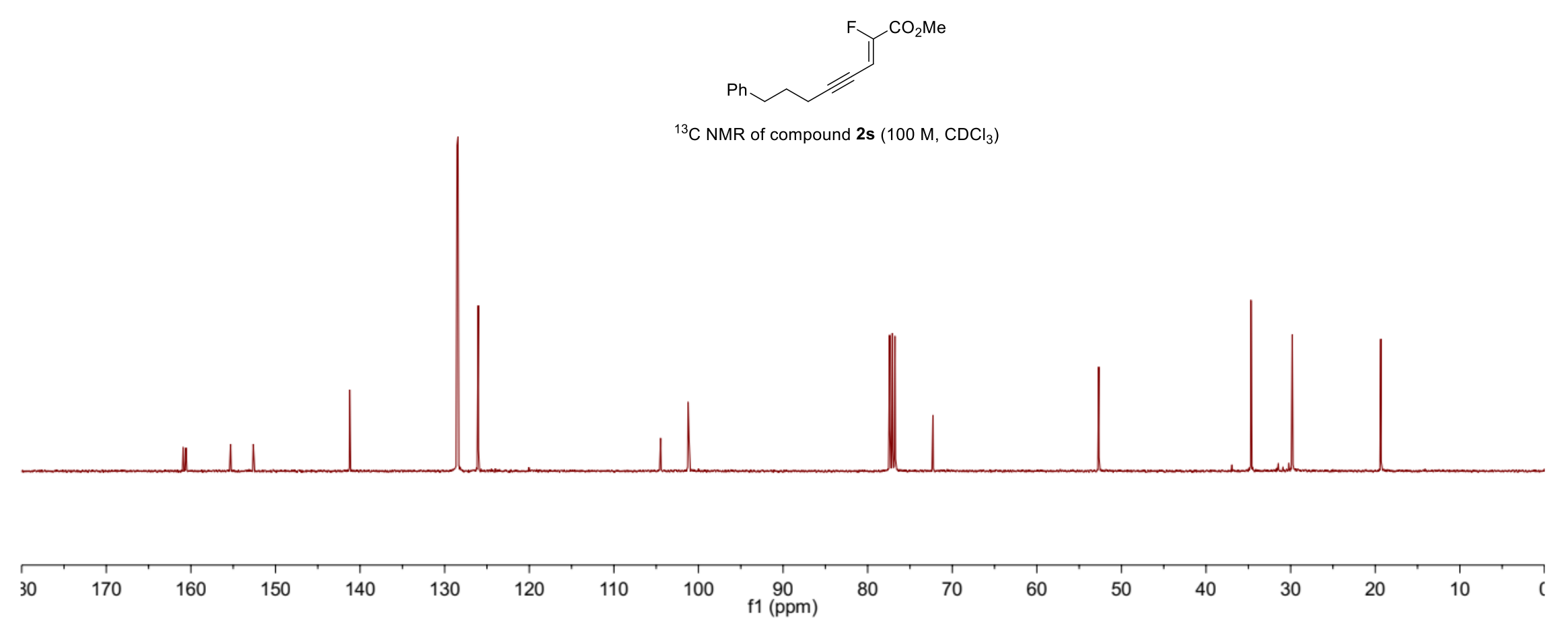




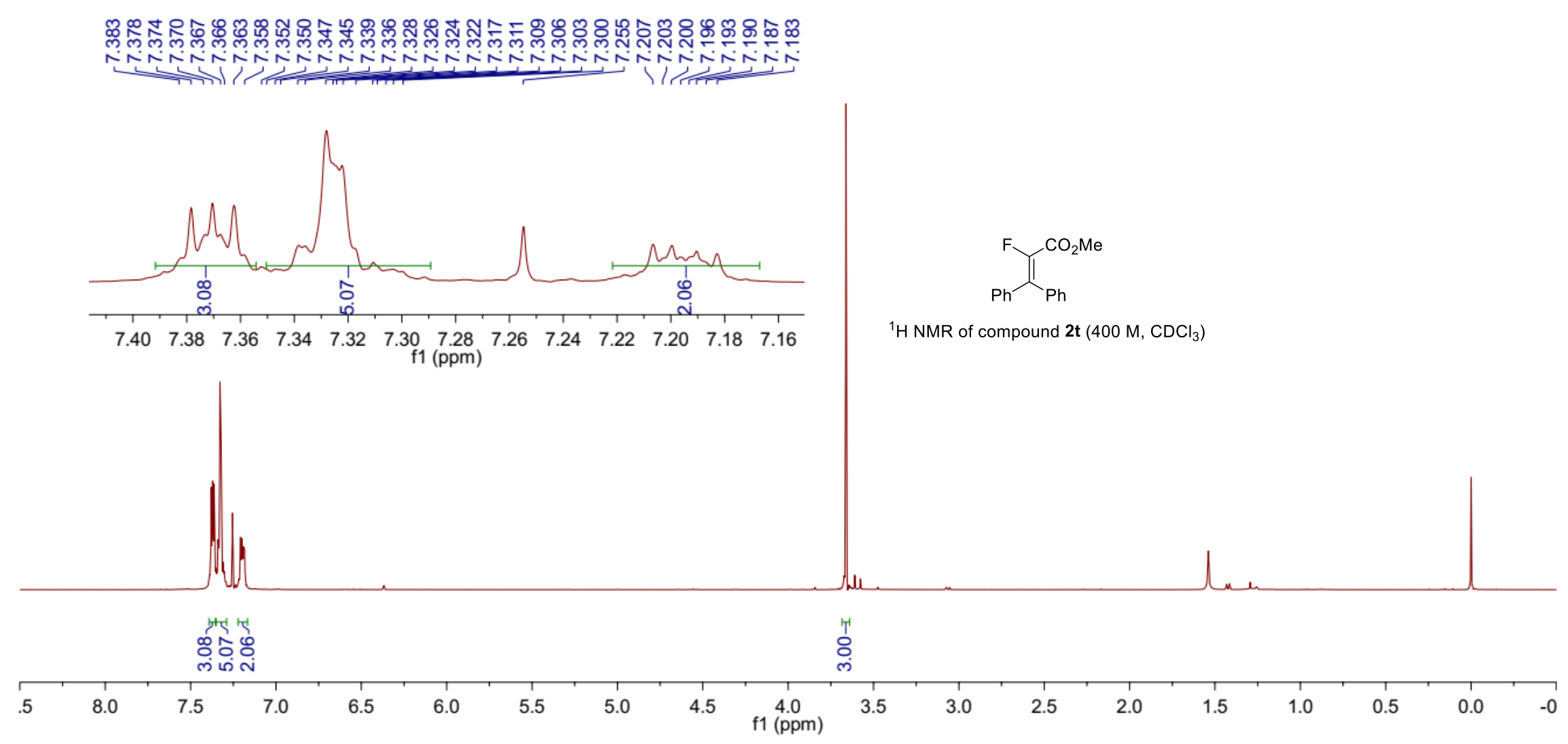


XSL-XJ-66-400M-2. 22. fid

XSL-XJ-66-400M-2 F

$\stackrel{\substack{\infty \\ \stackrel{i}{N}}}{\stackrel{1}{n}}$

$$
\overbrace{\mathrm{Ph}}^{\mathrm{CO}_{2} \mathrm{Me}}
$$

${ }^{19} \mathrm{~F} \mathrm{NMR}$ of compound $\mathbf{2 t}\left(376 \mathrm{M}, \mathrm{CDCl}_{3}\right)$

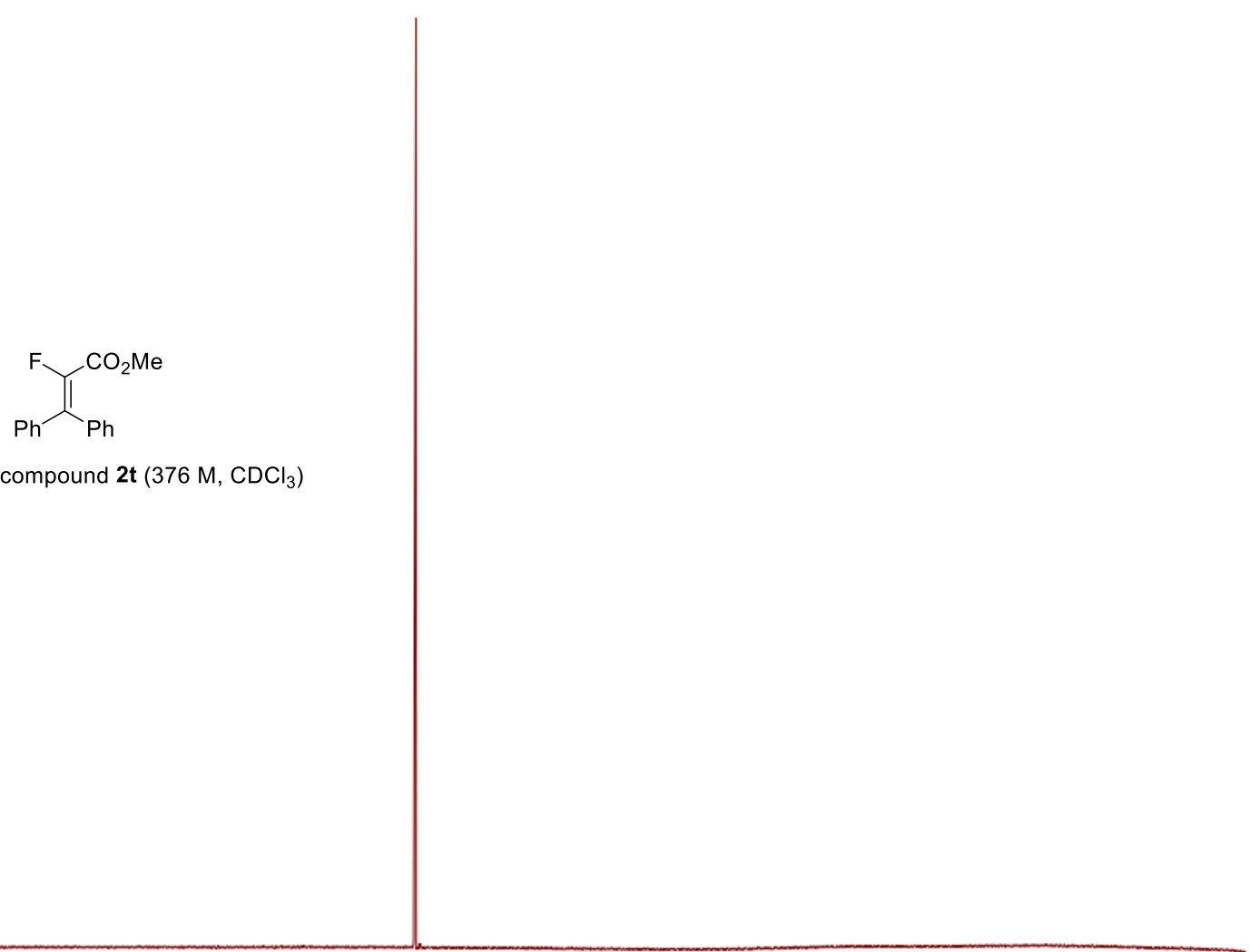

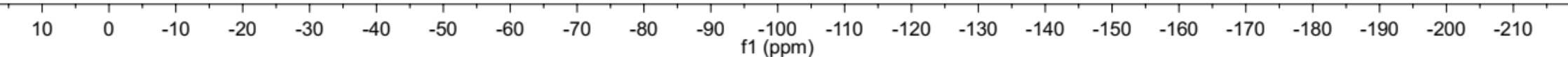


XSL-XJ-66-400MC $-10 \mathrm{fid}$ XSL-XJ-66-400MC N

ปั่

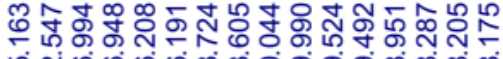

守

1

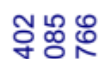

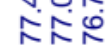

Nin

ลิ ลิ

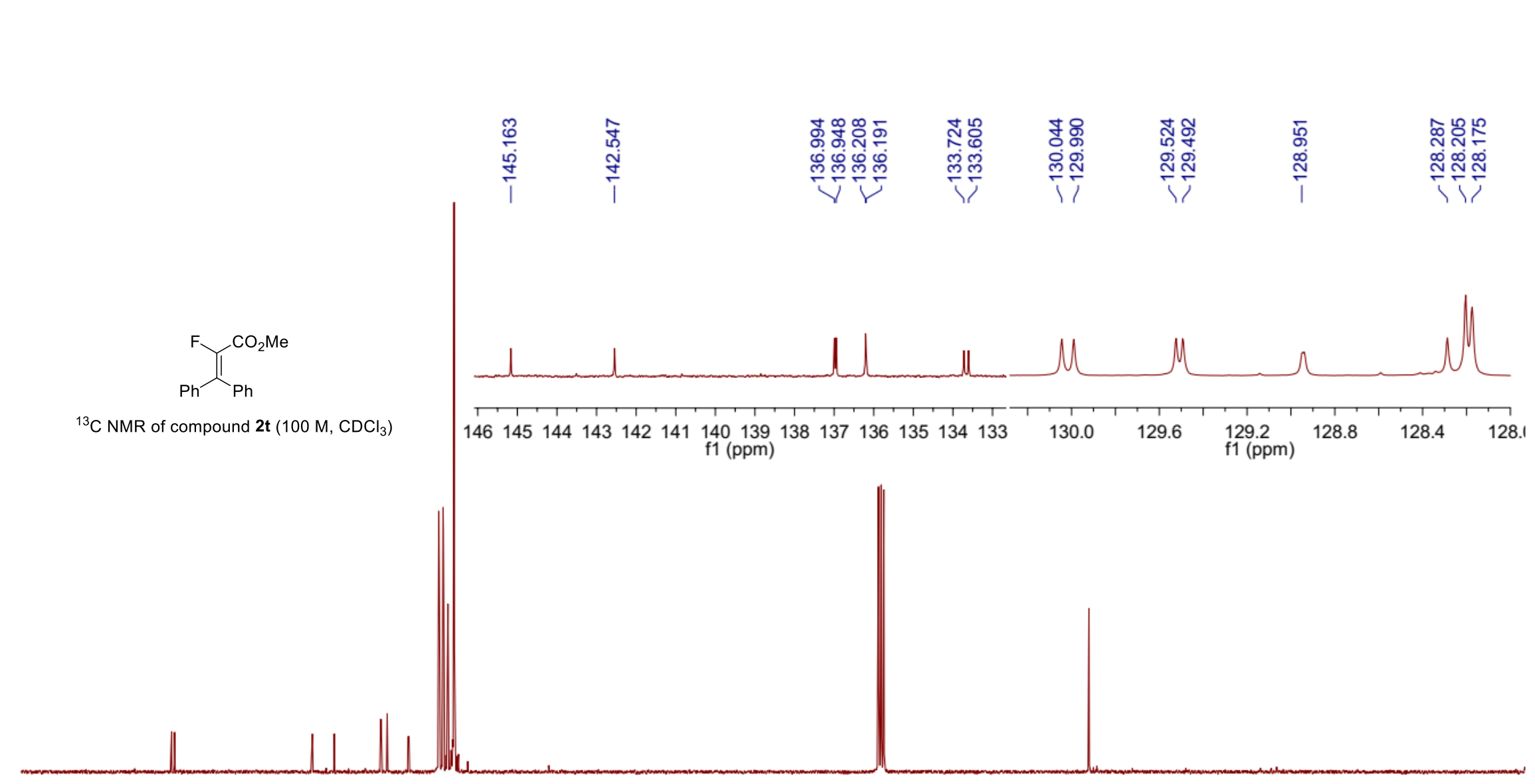$$
30
$$

$170 \quad 160 \quad 150$
$30 \quad 140$

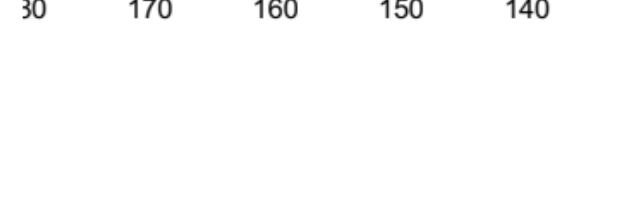

${ }^{13} \mathrm{C}$ NMR of compound $2 \mathbf{t}\left(100 \mathrm{M}, \mathrm{CDCl}_{3}\right)$

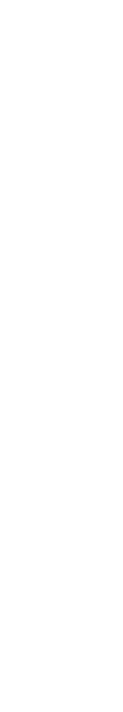

$\begin{array}{llllll}30 & 170 & 160 & 150 & 140 & 130\end{array}$

î.

.

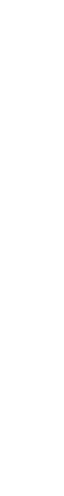




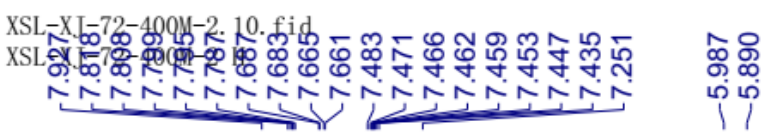
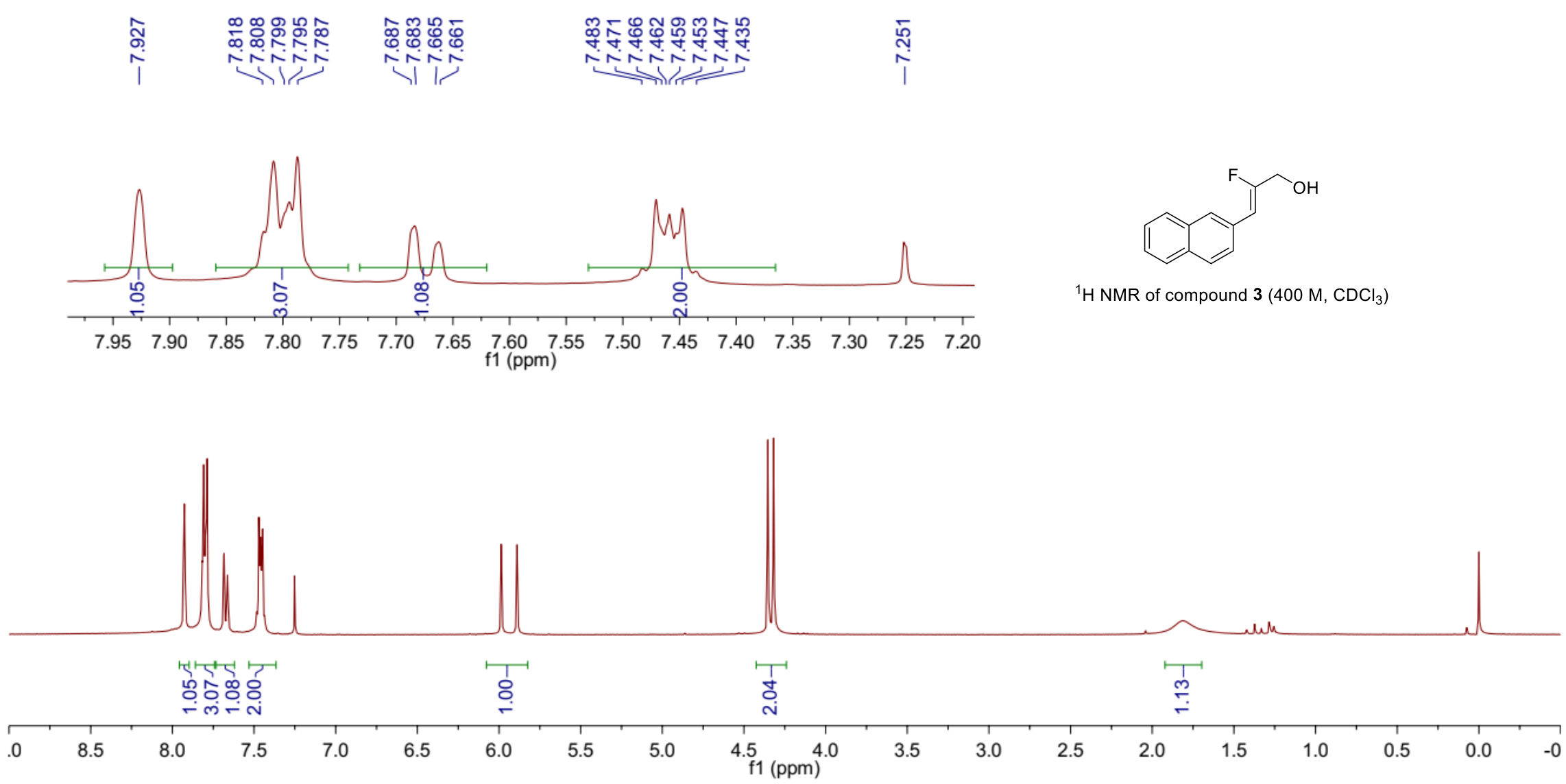
XSL-XJ-72-400M-2. 11. fid

XSL-XJ-72-400M-2 F

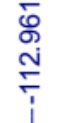

${ }^{19} \mathrm{~F} \mathrm{NMR}$ of compound $3\left(376 \mathrm{M}, \mathrm{CDCl}_{3}\right)$

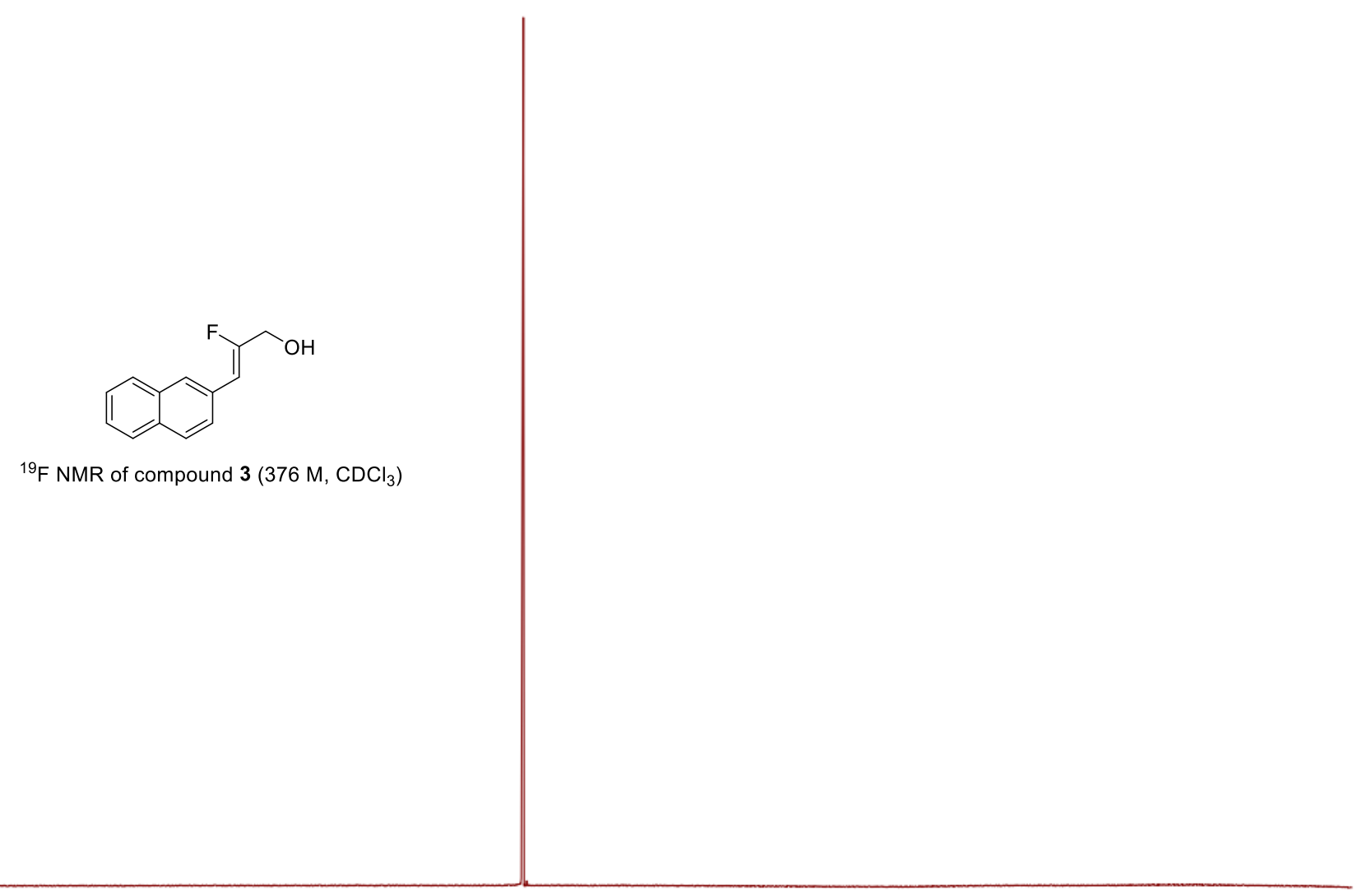

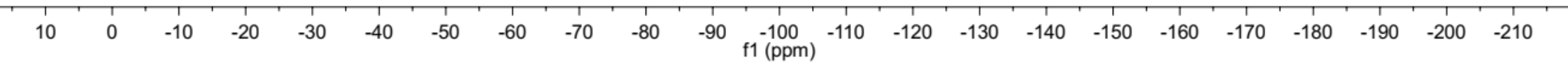




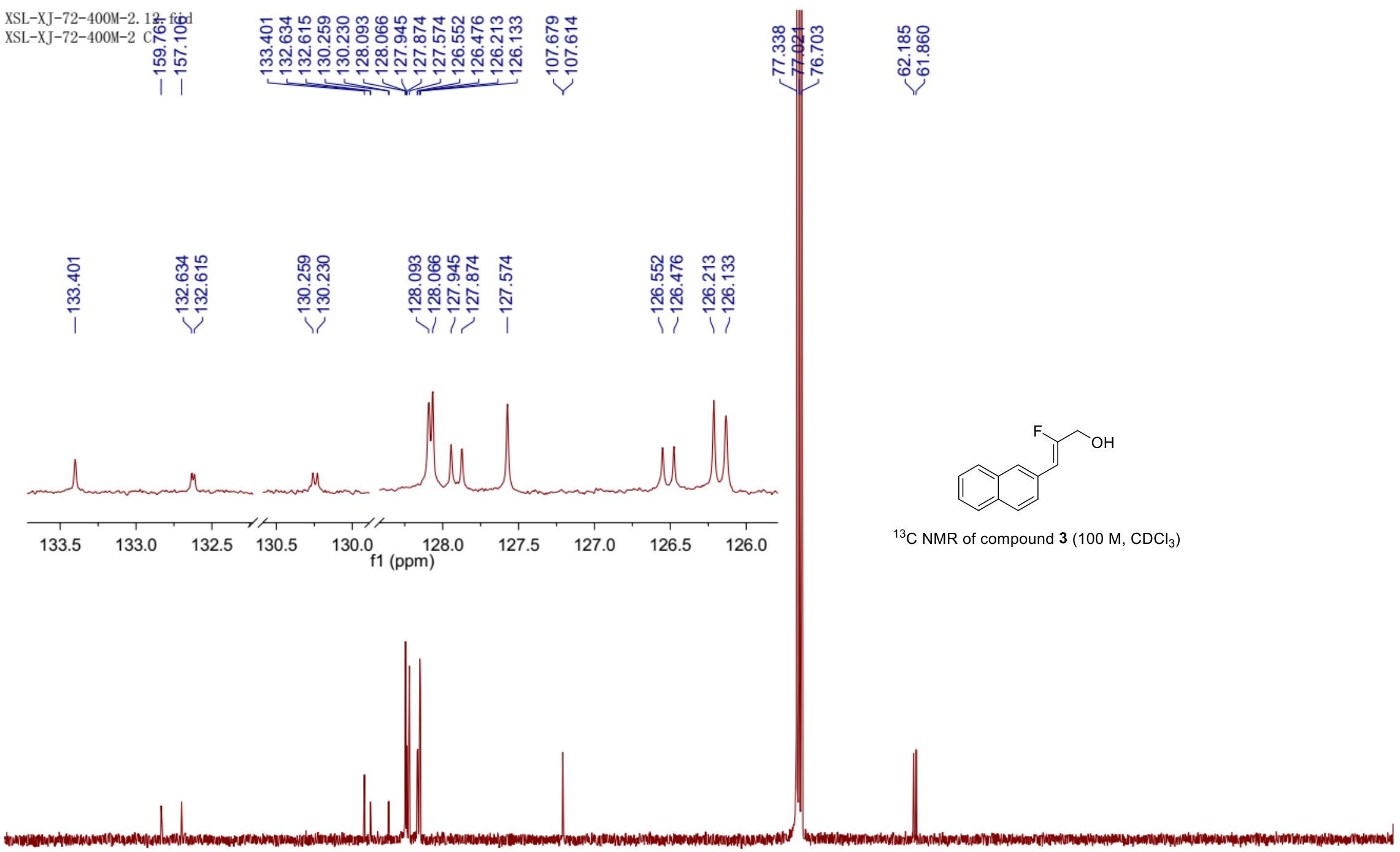

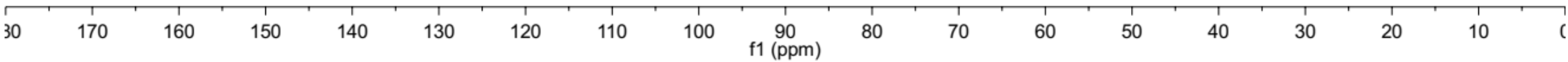




\section{(1)}

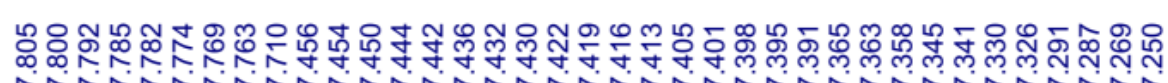

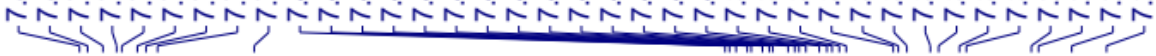

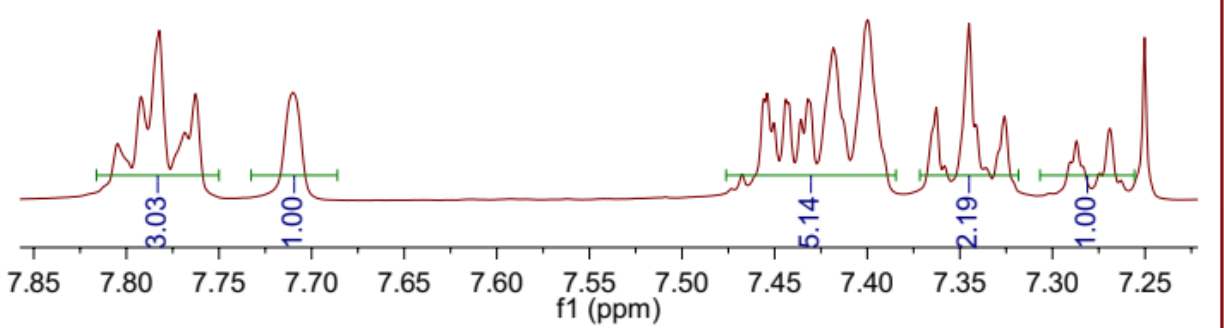

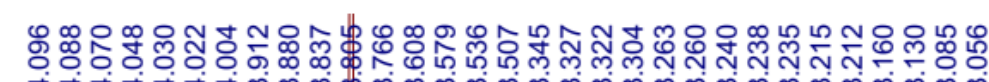

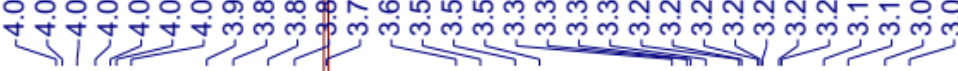
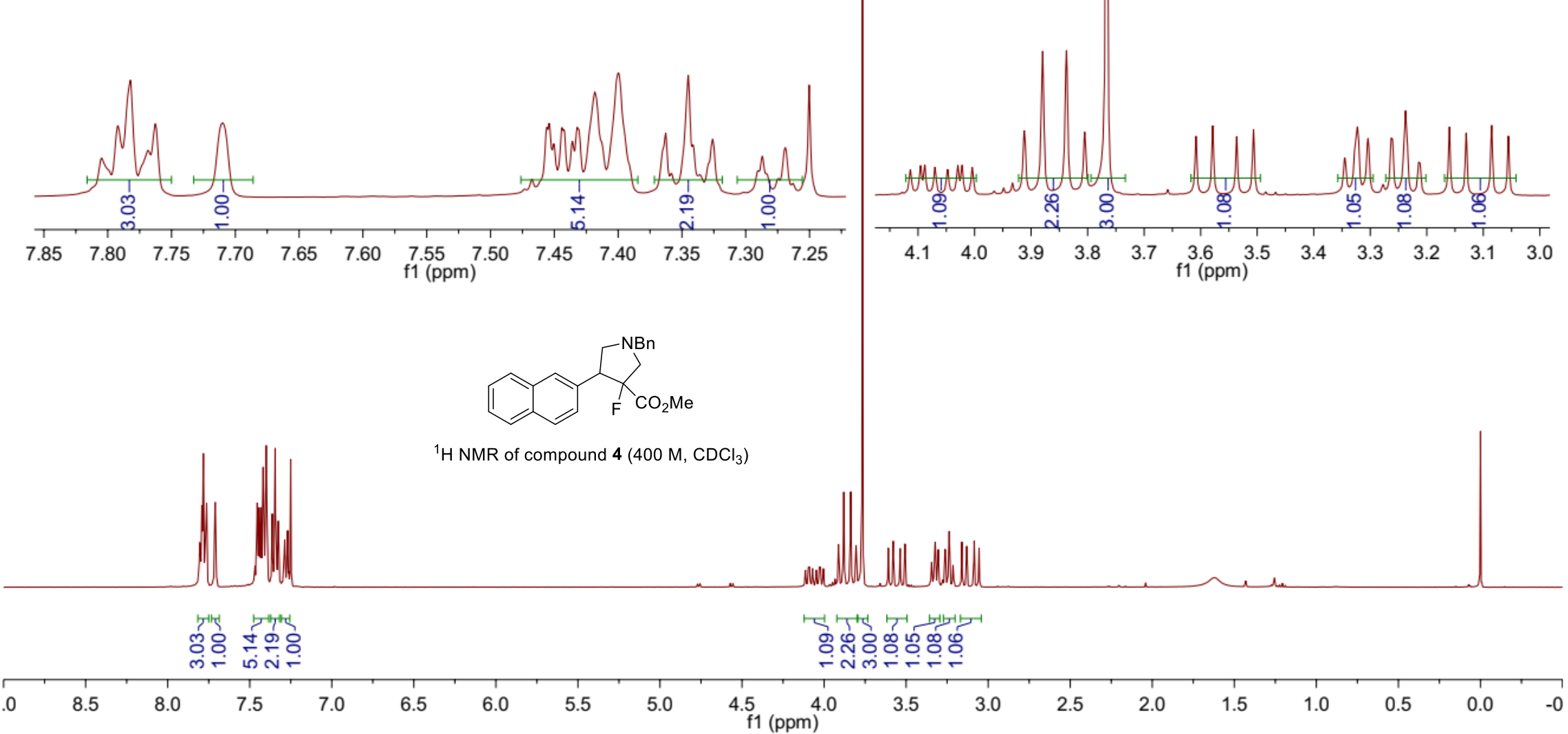
XSL-XJ-71-400M-2. 11. fid

XSL-XJ-71-400M-2 F

${ }^{19} \mathrm{~F} \mathrm{NMR}$ of compound $4\left(376 \mathrm{M}, \mathrm{CDCl}_{3}\right)$

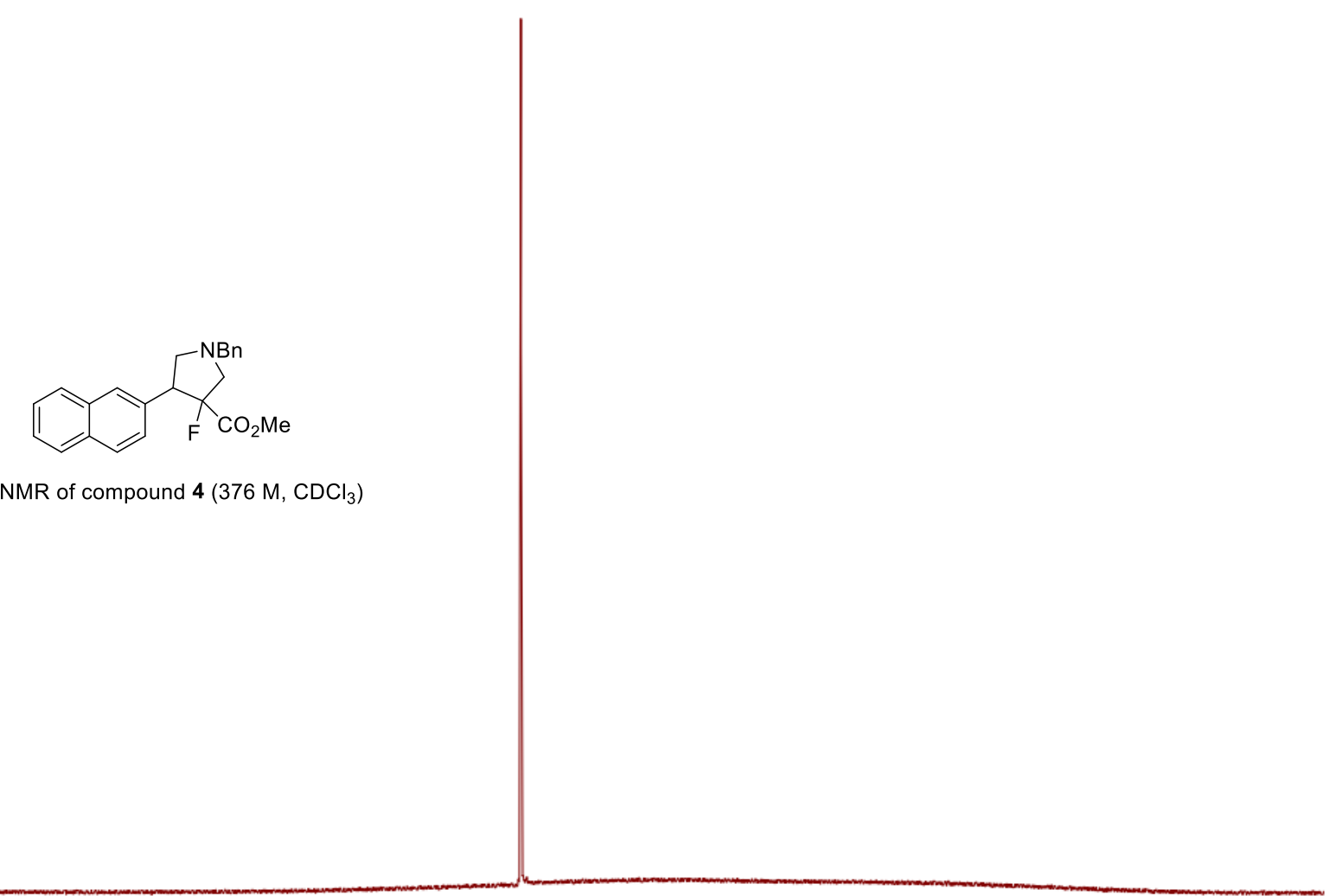

กั้

$\stackrel{\circ}{1}$

\begin{tabular}{lllllllllllllllllllllllllllllllllllllll}
\hline 0 & -95 & -100 & -105 & -110 & -115 & -120 & -125 & -130 & -135 & -140 & -145 & -150 & -155 & -160 & -165 & -170 & -175 & -180 & -185 & -190 & -195 & -200 & -205 & -2
\end{tabular} 


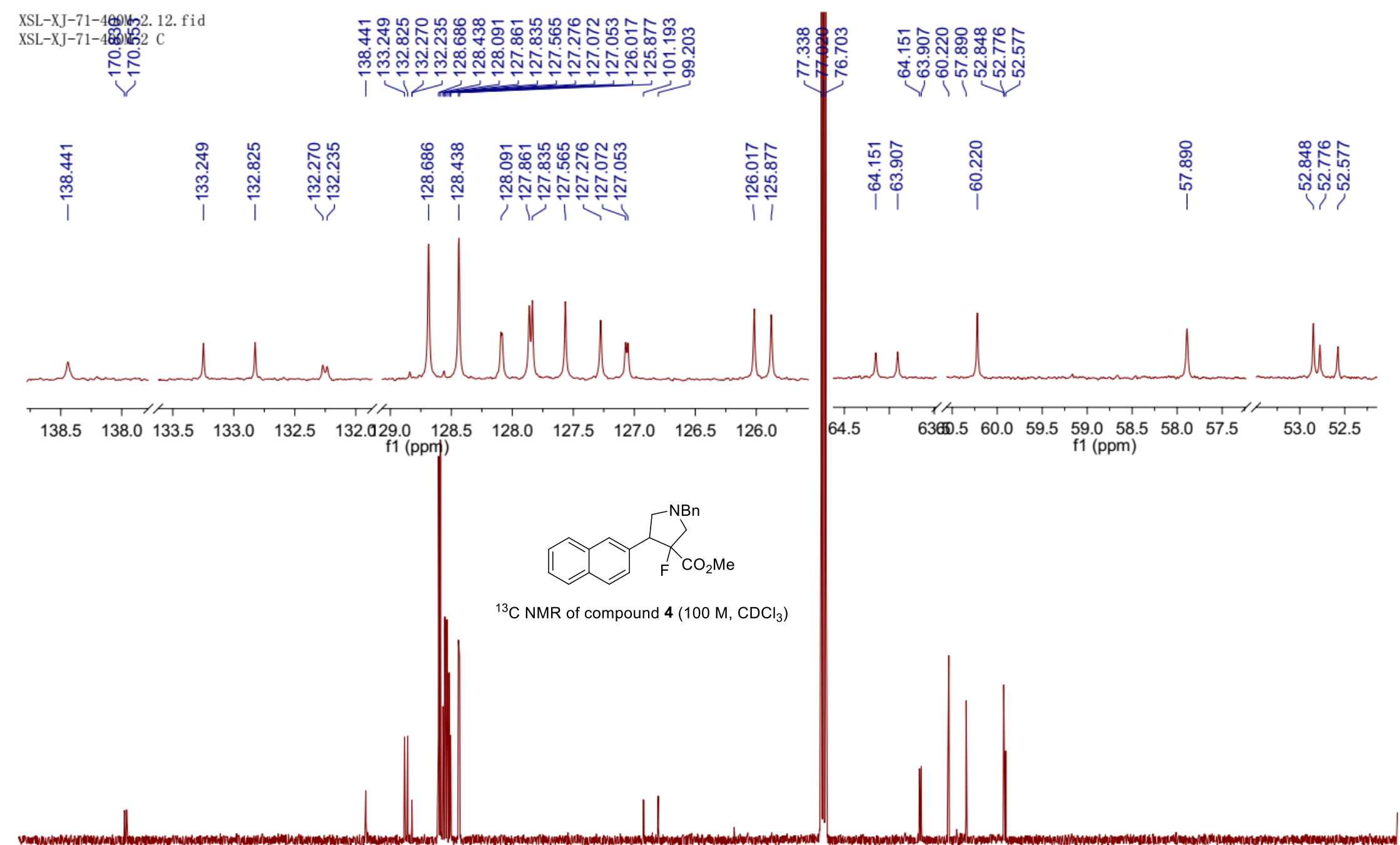

\begin{tabular}{|c|c|c|c|c|c|c|c|c|c|c|c|c|c|c|c|c|c|}
\hline 180 & 170 & 160 & 150 & 140 & 130 & 120 & 110 & 100 & $\begin{array}{r}90 \\
\mathrm{f} 1(\mathrm{ppm})\end{array}$ & 80 & 70 & 60 & 50 & 40 & 30 & 20 & 10 \\
\hline
\end{tabular}

